\title{
THE TWISTED TENSOR L-FUNCTION OF GSP(4)
}

\author{
DISSERTATION
}

\author{
Presented in Partial Fulfillment of the Requirements for \\ the Degree Doctor of Philosophy in the Graduate \\ School of the Ohio State University
}

\author{
By \\ Justin Young, B.S. \\ $* * * * *$ \\ The Ohio State University \\ 2009
}

\section{Dissertation Committee:}

Professor Stephen Rallis, Advisor

Professor James Cogdell

Professor Cary Rader
Approved by

Advisor

Graduate Program in Mathematics 



\begin{abstract}
We construct an integral representation for the "twisted tensor" $L$-function of a globally generic cuspidal automorphic representation of $\mathrm{GSp}_{4}$ over a number field. We prove that the integral is Eulerian, i.e., has an infinite product expansion. We compute the unramified integrals and show by way of a branching result (from $\mathrm{GL}_{4}$ to $\left.\mathrm{Sp}_{4}\right)$ that these integrals calculate the correct local $L$-factor. This gives a new proof of the analogous identity in D. Jiang's thesis. Finally, we show all the local integrals are absolutely convergent in a right half-plane and that they are nonvanishing for appropriate choice of data. We close with some remarks about poles of our global integral and possible future applications to period integrals and quadratic base change for $\mathrm{GSp}_{4}$.
\end{abstract}


To my wonderful wife, Dotty, for her love and consistent encouragement, and to my girls, Lily and Eva, for reminding me what it's all about. 


\section{ACKNOWLEDGMENTS}

First and foremost, I would like to thank my advisor, Professor Steve Rallis, for introducing me to the study of automorphic forms and for his genuine interest in my professional development. You have been readily available to discuss any questions of research or other aspects of the mathematics profession, and I appreciate it greatly.

Second, I would like to thank Professor Jim Cogdell for always having his door open to answer my questions, technical and otherwise, and for regularly helping me orient myself among the myriad ideas that enter the study of this subject. Thanks too for your understanding and willingness to work within my triple constraints of graduate student, husband and father.

I would also like to say thanks to Professor Cary Rader for freely sharing your experience and expertise and serving on my committee, and Chris Zorn for extensive feedback on this manuscript. Special thanks go to Dihua Jiang for his invaluable feedback, ideas and encouragement in this project.

I have had many teachers at Ohio State who modeled excellence in research and teaching to me, but few better than Professor Jeff McNeal, whose clear exposition and encouragement opened up a subject I previously thought impenetrable.

Lastly, I'd like to thank my fellow students, Yusuf Danisman, Dan File, Michael 
Khoury, Sheng-Chi Liu and Martin Nikolov, and my good friend Liang Niu for many stimulating and enjoyable conversations during my time here at Ohio State. 


\section{VITA}

2008-Present ..................... Graduate Teaching Associate, The Ohio State University

$2002-2008$ VIGRE Graduate Associate, The Ohio State University

2002 B.S. in Mathematics, B.A. in Physics, University of Kentucky

\section{FIELDS OF STUDY}

Major Field: Mathematics

Specialization: Automorphic Forms, $L$-functions 


\section{TABLE OF CONTENTS}

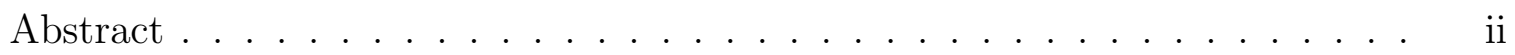

Dedication .............................. iii

Acknowledgments . . . . . . . . . . . . . . . . . . iv

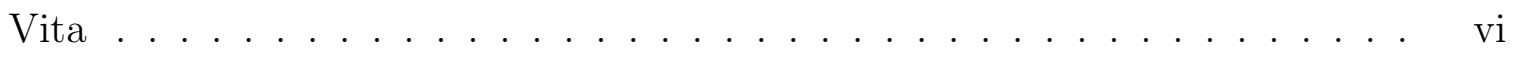

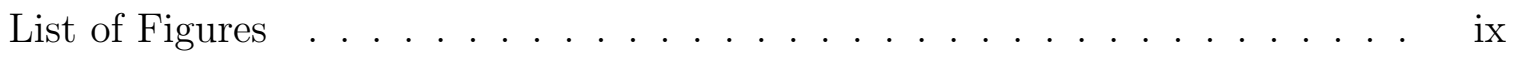

CHAPTER PAGE

$1 \quad$ Introduction . . . . . . . . . . . . . . . . . 1

$2 \quad$ Global Integral $\ldots \ldots \ldots \ldots$

$2.1 \quad$ Preliminaries . . . . . . . . . . . . . 6

$2.2 \quad$ Roots, Weights and Subgroups . . . . . . . . . 10

$2.3 \quad$ Orbit Decomposition . . . . . . . . . . . . 14

$2.4 \quad$ A Lemma on Cusp Forms . . . . . . . . . . . . . 16

$2.5 \quad$ The Global Integral $\ldots \ldots \ldots \ldots$

$3 \quad$ Unramified Computation . . . . . . . . . . . . . . . . . 26

$3.1 \quad$ Inert Case . . . . . . . . . . . . . . 26

3.1.1 The Inner Integral $I\left(t, f_{s}^{\circ}, \psi\right) \ldots \ldots \ldots \ldots \ldots \ldots$

3.1 .2 The Unramified Integral $\ldots \ldots \ldots . \ldots \ldots$

3.1 .3 Local $L$-factor . . . . . . . . . . . . . . . . . . . . . . 45

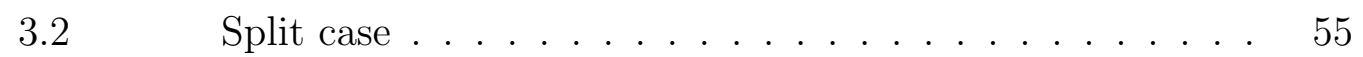

3.2 .1 The Split Integral . . . . . . . . . . . . . . . . . . . . 56

$3.2 .2 \quad$ Local $L$-factor . . . . . . . . . . . . . . . . . . . . . . 63

$3.3 \quad$ Proof of combinatorial lemmas . . . . . . . . 67 
$4 \quad$ Analysis of Local Integrals . . . . . . . . . . . . . . . . . 94

$4.1 \quad$ Absolute Convergence . . . . . . . . . . . . . . 95

4.1.1 Nonarchimedean integrals . . . . . . . . . . . . . . . 100

4.1.2 Archimedean integrals . . . . . . . . . . . . . . . . . 102

$4.2 \quad$ Nonvanishing . . . . . . . . . . . . . . . . 104

4.2.1 Nonarchimedean case . . . . . . . . . . . . . . . . 107

4.2.2 Archimedean case . . . . . . . . . . . . . . . . . . . . . . . . . 119

4.3 Final Remarks . . . . . . . . . . . . . . . . 123

Bibliography . . . . . . . . . . . . . . . . . . . . 128 


\section{LIST OF FIGURES}

FIGURE PAGE

3.1 A Young diagram of shape $(6,4,4,1) \ldots \ldots$. . . . . . . . 49

3.2 A column-strict Young tableau . . . . . . . . . . . . . . . . 49

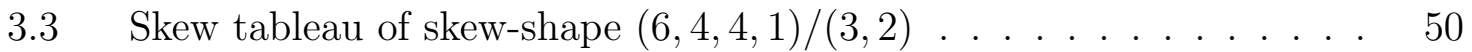

3.4 The partitions $\lambda, \beta$ and $\mu \ldots \ldots \ldots$. . . . . . . . . . . . . .

3.5 The partitions $\lambda, \alpha$ and $\nu \ldots \ldots \ldots \ldots$. . . . . . . . . . . . . . 


\section{CHAPTER 1 INTRODUCTION}

The $L$-function of an automorphic representation, though defined in terms of essentially local data, carries a great deal of global information about the representation. For example, the presence of a pole of some order at $s=1$ may indicate that the representation in question is a functorial lift from an automorphic representation on another group. Several such cases have been established; see [25] for a recent example. It is our purpose here to lay the groundwork for a new case, that of the twisted tensor $L$-function for $\mathrm{GSp}_{4}$ as it relates to detecting quadratic base change lifts. We do this by constructing an integral representation for this $L$-function.

Given an integral representation of an $L$-function, one obtains analytic information about the $L$-function in the following way. The $L$-function depends on a complex variable $s$, and this dependence enters the integral by way of some function in the integrand, usually an Eisenstein series. The integral will usually be convergent in a right half-plane (i.e. for $\operatorname{Re}(s)$ sufficiently large), and then the analytic properties of the Eisenstein series propagates to the $L$-function.

Though our construction is one obvious analogue to D. Jiang's thesis [24], its origins (as well as those of Jiang's work) lie in the theory of integral representations of $L$-functions for $\mathrm{GL}_{n} \times \mathrm{GL}_{n}$. We will briefly recall this and several other related 
constructions. For the theory on $\mathrm{GL}_{n} \times \mathrm{GL}_{n}$, the author greatly benefited from the exposition in [6], where he first learned this material.

The theory of integral representations for $\mathrm{GL}_{n} \times \mathrm{GL}_{m}$ was developed by H. Jacquet, I. Piatetski-Shapiro and J. Shalika in a series of papers $([17,18,23,22,19,21]$; see also [7]); here we consider the case $m=n$. Let $\pi$ and $\pi^{\prime}$ be smooth cuspidal automorphic representations of $\mathrm{GL}_{n}(\mathbb{A})$, where $\mathbb{A}$ is the adele ring of a number field $F$. Let $\varphi \in V_{\pi}$ and $\varphi^{\prime} \in V_{\pi^{\prime}}$ be cusp forms. Then there is an Eisenstein series $E_{n}(g, s)$ on $\mathrm{GL}_{n}(\mathbb{A})$, given explicitly in terms of a theta series, such that the integral

$$
I\left(s, \varphi, \varphi^{\prime}, E\right)=\int_{Z_{n}(\mathbb{A}) \mathrm{GL}_{n}(F) \backslash \mathrm{GL}_{n}(\mathbb{A})} \varphi(g) \varphi^{\prime}(g) E_{n}(g, s) d g
$$

represents the product (or Rankin-Selberg) $L$-function $L\left(s, \pi \times \pi^{\prime}\right)$. (See [6, Lec. 9], [7].)

Jiang's construction enters the picture through an "accidental isomorphism" and the theory of seesaw dual reductive pairs [30]. The original motivation for his work comes from questions of functoriality and theta liftings; see the introduction to [24] for a discussion of this. The integral representation arises as follows. Consider the integral $(*)$ in the case $n=4$. Since $\mathrm{PGL}_{4} \simeq \mathrm{PGO}_{3,3}$, this can be thought of as an integral of cusp forms and Eisenstein series on $\mathrm{GO}_{3,3}$ :

$$
\int_{Z_{3,3}(\mathbb{A}) \mathrm{GO}_{3,3}(F) \backslash \mathrm{GO}_{3,3}(\mathbb{A})} \phi(g) \phi^{\prime}(g) E_{3,3}(g, s) d g .
$$

Now this integral can be interpreted as half of a seesaw identity (in the sense of [30]), 
where the dual reductive pairs in question are given by the diagram

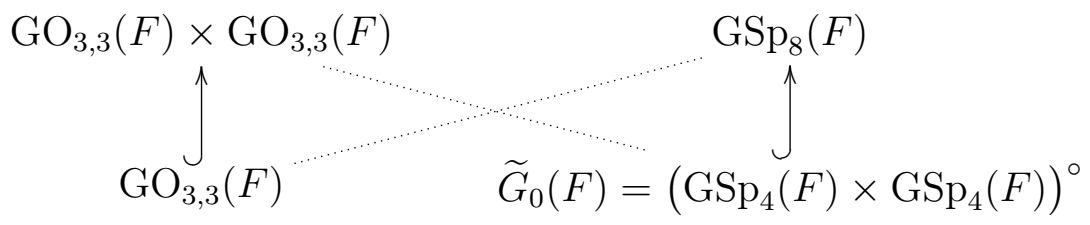

where the left-hand inclusion is the diagonal embedding and the right-hand inclusion is the doubling embedding (see [35] and Section 2.1 of this paper), and

$$
\left(\operatorname{GSp}_{4}(F) \times \operatorname{GSp}_{4}(F)\right)^{\circ}=\left\{\left(g_{1}, g_{2}\right) \mid g_{1}, g_{2} \in \operatorname{GSp}_{4}(F), \lambda\left(g_{1}\right)=\lambda\left(g_{2}\right)\right\}
$$

where $\lambda$ is the similitude character. The seesaw identity then reads

$$
\begin{aligned}
\int_{Z_{3,3}(\mathbb{A}) \mathrm{GO}_{3,3}(F) \backslash \mathrm{GO}_{3,3}(\mathbb{A})} \phi(g) \phi^{\prime}(g) E_{3,3}(g, s) d g & \\
& =\int_{Z(\mathbb{A}) \widetilde{G}_{0}(F) \backslash \widetilde{G}_{0}(\mathbb{A})} \tilde{\phi}\left(g_{1}\right) \tilde{\phi}^{\prime}\left(g_{2}\right) \tilde{E}_{8}\left(\left(g_{1}, g_{2}\right), s\right) d g,
\end{aligned}
$$

where $\tilde{\phi}$ and $\tilde{\phi}^{\prime}$ are the theta lifts of $\phi$ and $\phi^{\prime}$, respectively, from $\operatorname{GO}_{3,3}(\mathbb{A})$ to $\operatorname{GSp}_{4}(\mathbb{A})$, and $\tilde{E}_{8}$ is the theta lift of the Eisenstein series $E_{3,3}$ from $\mathrm{GO}_{3,3}(\mathbb{A})$ to $\mathrm{GSp}_{8}(\mathbb{A})$, given by the regularized theta integral of S. Kudla and S. Rallis [31]. Using the appropriate choice for $\tilde{E}_{8}$, Jiang shows this integral represents the Rankin-Selberg $L$-function for the product of two (generic) cuspidal automorphic representations of $\mathrm{GSp}_{4}$. These relations can be loosely summarized by the diagram:

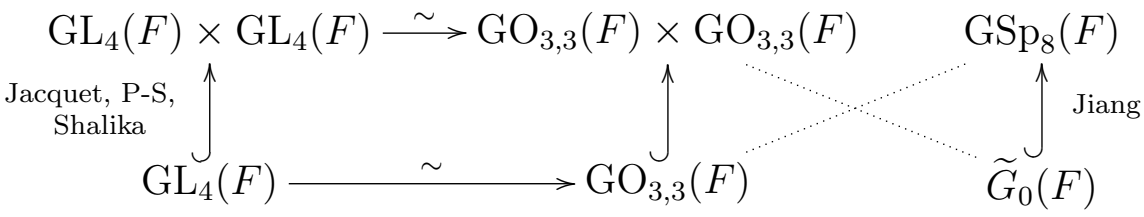

One natural generalization of these construction is to consider $G(F) \times G(F)$ as $G(F \oplus$ $F)$, and ask whether the construction can be repeated when the quadratic algebra 
$F \oplus F$ is replaced by a quadratic extension $E$ of $F$. The answer for $G=\mathrm{GL}_{n}$ is affirmative and was given by Y. Flicker in [8]. His integral is analogous to $(*)$ :

$$
I\left(s, \varphi, E_{n}\right)=\int_{Z_{n}\left(\mathbb{A}_{F}\right) \mathrm{GL}_{n}(F) \backslash \mathrm{GL}_{n}\left(\mathbb{A}_{F}\right)} \varphi(g) E_{n}(g, s) d g,
$$

where $\varphi$ is now a cuspidal automorphic form on $\mathrm{GL}_{n}\left(\mathbb{A}_{E}\right)$ and $E_{n}$ is the same Eisenstein series as in $(*)$. Flicker shows that the integral represents the "twisted tensor" $L$-function (in some contexts, also called the Asai $L$-function) for the cuspidal representation containing $\varphi$.

Can the seesaw construction be repeated in this context to give another integral representation, this time on $\mathrm{GSp}_{4}(E)$ ? The point of this dissertation is to answer in the affirmative. The situation for $E / F$ a quadratic extension can be represented by the diagram

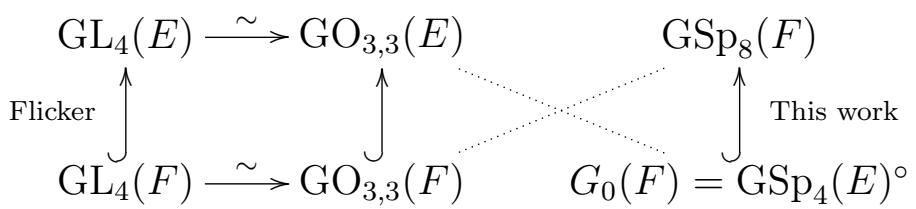

where $\mathrm{GSp}_{4}(E)^{\circ}=\left\{g \in \mathrm{GSp}_{4}(E) \mid \lambda(g) \in F^{\times}\right\}$.

Now we will briefly describe the contents of this work. In the second chapter, after giving the notation and basic setup for our integral, we carry out an orbit decomposition and a establish a technical result on cusp forms to prove that the integral is Eulerian. The third chapter is the calculation of the unramified integrals and identification of these with the correct local $L$-factor. The method of this identification is new: Jiang uses the Kostant-Rallis algorithm in [24], but here we use a branching rule from $\mathrm{GL}_{4}$ to $\mathrm{Sp}_{4}$, which gives a new proof of Jiang's identity for places $v$ of $F$ 
which are split in E. Finally, the fourth chapter carries out some analyses of the local integrals; we show these are absolutely convergent in a right half-plane and that they are nonvanishing for appropriate choice of data. Then we close with some remarks about poles of our global integral and possible future applications. 


\section{CHAPTER 2}

\section{GLOBAL INTEGRAL}

\section{$2.1 \quad$ Preliminaries}

Let us first give the main notations of the work.

Let $E / F$ be a quadratic extension of number fields generated by an algebraic integer $\theta$ with $\theta^{2} \in F$, and let $\mathbb{A} \stackrel{\text { def }}{=} \mathbb{A}_{F}$ be the adéle ring of $F$. Our construction involves the groups of symplectic similitudes,

$$
\mathrm{GSp}_{2 n}=\left\{g \in \mathrm{GL}_{2 n} \mid g J_{2 n}{ }^{t} g=\lambda J_{2 n}\right\}
$$

where $J_{2 n}=\left(\begin{array}{cc}0 & I_{n} \\ -I_{n} & 0\end{array}\right)$ and $\lambda=\lambda(g)$ is the similitude character. We get embeddings between these groups by considering a "twisted" variation of the usual doubling method of Piatetski-Shapiro and Rallis [35].

Let $V$ be a $2 n$-dimensional vector space over $E$ with nondegenerate symplectic form $\langle\cdot, \cdot\rangle$, and let $W=\operatorname{Res}_{E / F} V$ with form $\langle\langle x, y\rangle\rangle=\frac{1}{2} \operatorname{Tr}_{E / F}\langle x, y\rangle$, which is also symplectic. Denote the similitude groups of these forms by $\operatorname{GSp}(V)$ and $\operatorname{GSp}(W)$, respectively. Notice that if $g \in \operatorname{GSp}(V)$ has similitude $\lambda(g) \in F$, then for $v, w \in V$,

$$
\begin{aligned}
\langle\langle v g, w g\rangle\rangle & =\operatorname{Tr}_{E / F}\langle v g, w g\rangle=\operatorname{Tr}_{E / F}(\lambda(g)\langle v, w\rangle) \\
& =\lambda(g)\langle\langle v, w\rangle\rangle
\end{aligned}
$$


so we get an embedding $\operatorname{GSp}(V)^{\circ} \hookrightarrow \operatorname{GSp}(W)$, where ${ }^{\circ}$ denotes the subgroup of elements whose similitudes lie in $F$. After fixing a basis for $V$ and $W$ this becomes $\mathrm{GSp}_{2 n}(E)^{\circ} \hookrightarrow \operatorname{GSp}_{4 n}(F)$.

We consider the case $n=2$. Namely, $V$ is 4-dimensional over $E$, with nondegenerate symplectic form $\langle\cdot, \cdot\rangle$ and standard basis $\left\{e_{1}, e_{2}, e_{1}^{\prime}, e_{2}^{\prime}\right\}$, so that $\left\langle e_{i}, e_{j}^{\prime}\right\rangle=\delta_{i j}$ and $\left\langle e_{i}, e_{j}\right\rangle=\left\langle e_{i}^{\prime}, e_{j}^{\prime}\right\rangle=0$. Let $W=\operatorname{Res}_{E / F} V$ with form $\langle\langle x, y\rangle\rangle=\frac{1}{2} \operatorname{Tr}_{E / F}\langle x, y\rangle$. Denote

$$
G_{0}(F) \stackrel{\text { def }}{=} \operatorname{GSp}_{4}(E)^{\circ}=\left\{g \in \operatorname{GSp}_{4}(E) \mid \lambda(g) \in F\right\}
$$

(Alternatively, $G_{0}$ can be described as a fibre product:

$$
G_{0}=\operatorname{Res}_{E / F} \mathrm{GSp}_{4} \times_{\operatorname{Res}_{E / F} \mathbb{G}_{m}} \mathbb{G}_{m} \simeq\left(\operatorname{Res}_{E / F} \mathrm{Sp}_{4}\right) \rtimes \mathbb{G}_{m},
$$

where $\left.\mathrm{GSp}_{4}=\mathrm{Sp}_{4} \rtimes \mathbb{G}_{m}.\right)$ For later reference, we remark that $G_{0}$ is quasisplit over $F$

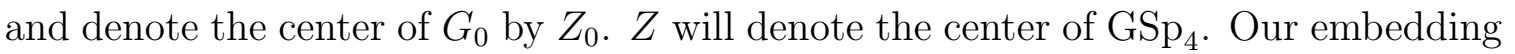
is $\iota: G_{0}(F) \hookrightarrow \operatorname{GSp}_{8}(F)$. Explicitly, fix the basis $\left\{e_{1}, \theta e_{1}, e_{2}, \theta e_{2}, e_{1}^{\prime}, \theta^{-1} e_{1}^{\prime}, e_{2}^{\prime}, \theta^{-1} e_{2}^{\prime}\right\}$ for $W$. Then $\iota$ is given by

$$
\iota:\left(\alpha_{i j}\right) \mapsto\left(\begin{array}{cc}
1 & \\
& T_{\theta}^{-1}
\end{array}\right)\left(m\left(\alpha_{i j}\right)\right)\left(\begin{array}{ll}
1 & \\
& \\
& T_{\theta}
\end{array}\right),
$$

where

$$
\alpha=a+a^{\prime} \theta \mapsto m(\alpha)=\left(\begin{array}{cc}
a & a^{\prime} \\
a^{\prime} \theta^{2} & a
\end{array}\right)
$$

is the usual embedding $E \hookrightarrow \mathrm{GL}_{2}(F)$, and

$$
T_{\theta}=\operatorname{diag}\left(1, \theta^{2}, 1, \theta^{2}\right)
$$


For convenience, we will identify an element of $G_{0}(F)$ with its image in $\operatorname{GSp}_{8}(F)$. In the classical doubling method [35], one takes cusp forms $\varphi_{1}$ and $\varphi_{2}$ on $\operatorname{Sp}_{2 n}(\mathbb{A})$ and integrates against a Siegel-type Eisenstein series on $\operatorname{Sp}_{4 n}(\mathbb{A})$ over the diagonally embedded copy of $\operatorname{Sp}_{2 n}(\mathbb{A}) \times \operatorname{Sp}_{2 n}(\mathbb{A})$. (For the similitude case, one can only embed the subgroup

$$
\left(\operatorname{GSp}_{2 n}(\mathbb{A}) \times \operatorname{GSp}_{2 n}(\mathbb{A})\right)^{\circ}=\left\{\left(g_{1}, g_{2}\right) \mid g_{1}, g_{2} \in \operatorname{GSp}_{2 n}(\mathbb{A}), \lambda\left(g_{1}\right)=\lambda\left(g_{2}\right)\right\}
$$

into $\operatorname{GSp}_{4 n}(\mathbb{A})$.) In our setup, we will take a cusp form $\varphi$ on $\operatorname{GSp}_{4}\left(\mathbb{A}_{E}\right)$ and a nonSiegel Eisenstein series on $\operatorname{GSp}_{8}\left(\mathbb{A}_{F}\right)$. (See the introduction for an explanation of this.)

Namely, $P_{8} \subseteq \mathrm{GSp}_{8}$ is the maximal parabolic subgroup stabilizing the isotropic 3plane spanned by $\left\{e_{1}^{\prime}, \theta^{-1} e_{1}^{\prime}, e_{2}^{\prime}\right\}$ :

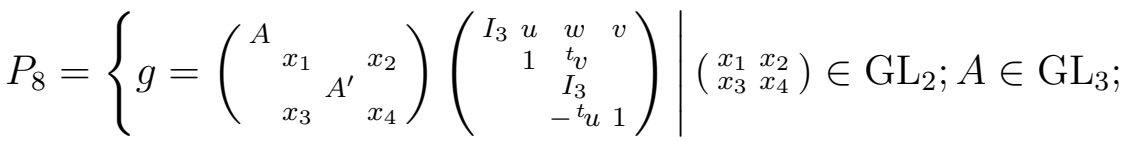

$$
\begin{aligned}
& \left.A^{\prime}=\left(x_{1} x_{4}-x_{2} x_{3}\right)^{t} A^{-1} ; u, v \in \mathrm{Mat}_{3,1} ; w \in \mathrm{Mat}_{3,3} ; w+v^{t} u \in \mathrm{Sym}_{3}\right\}
\end{aligned}
$$

The modulus character of $P_{8}$ at an element $g$ as above is

$$
\delta_{P_{8}}(g)=(\operatorname{det} A)^{6} \lambda(g)^{-9},
$$

and $\lambda(g)=x_{1} x_{4}-x_{2} x_{3}$. (By convention we write $\delta_{P_{8}}(g)^{s}=\left|(\operatorname{det} A)^{6} \lambda(g)^{-9}\right|^{s}$ for complex s.) Form the normalized induced representation

$$
\operatorname{Ind}_{P_{8}(\mathbb{A})}^{\mathrm{GSp}(\mathbb{A})}\left(\delta_{P_{8}}^{s / 6}\right)=\left\{f: \operatorname{GSp}_{8}(\mathbb{A}) \rightarrow \mathbb{C} \text { smooth } \mid f(p g)=\delta_{P_{8}}(p)^{\frac{1}{2}} \delta_{P_{8}}(p)^{\frac{s}{6}} f(g)\right\},
$$


and let $f_{s} \in \operatorname{Ind}_{P_{8}(\mathbb{A})}^{\mathrm{GSp}_{8}(\mathbb{A})}\left(\delta_{P_{8}}^{s / 6}\right)$ be a flat section, i.e., the restriction of $f_{s}$ to the maximal compact subgroup of $\mathrm{GSp}_{8}(\mathbb{A})$ is independent of $s$. Construct the Eisenstein series

$$
E\left(g, s, f_{s}\right)=\sum_{\gamma \in P_{8}(F) \backslash \mathrm{GSp}_{8}(F)} f_{s}(\gamma g) .
$$

This is identical to the Eisenstein series used by Jiang [24, II.2.2]. We must "normalize" this Eisenstein series in the following way. The constant term of the Eisenstein series is a sum of intertwining operators between various induced representations. Each of these operators is defined for $\operatorname{Re}(s)>>0$ and has a "denominator" in the sense that, after multiplying the operator by a certain (computable) product of zeta functions (the "denominator"), the operator has mermorphic continuation to the whole plane with a finite number of poles. The normalizing factor of the Eisenstein series is defined to be the LCM of these "denominators." Jiang computes the normalizing factor for $E\left(g, s, f_{s}\right)$ in [24], chapter III, Theorem 4.0.2 and preceding discussion. We only use the case $n=4$.

Lemma 2.1.1 ([24]). $d_{G}(s)=\prod_{v \notin S} \zeta_{v}(2 s+2) \zeta_{v}(s+1) \zeta_{v}(s+2) \zeta_{v}(s+3)$ is the normalizing factor for the Eisenstein series $E\left(g, s, f_{s}\right)$, where $S$ is the set of "bad" primes of $F$, to be defined later.

We define the normalized Eisenstein series $E^{*}\left(g, s, f_{s}\right)$ by

$$
E^{*}\left(g, s, f_{s}\right)=d_{G}(s) E\left(g, s, f_{s}\right)
$$

Proposition 2.1.2 ([33, 1]). The series $E^{*}\left(g, s, f_{s}\right)$ converges absolutely for $\operatorname{Re}(s)$ sufficiently large, has meromorphic continuation to the complex plane with finitely many poles, and satisfies a functional equation relating its values at $s$ and $-s$. 
Let $\pi$ be a smooth irreducible cuspidal automorphic representation of $\mathrm{GSp}_{4}\left(\mathbb{A}_{E}\right)$ which is globally generic and has central character $\omega_{\pi} \equiv 1$, and let $V_{\pi}$ be a realization of $\pi$ in the space of cusp forms on $\mathrm{GSp}_{4}\left(\mathbb{A}_{E}\right)$. By the embedding $\iota$, we may consider the restriction of $E\left(g, s, f_{s}\right)$ to $G_{0}$. We will examine the integral

$$
I\left(s, \varphi, f_{s}\right)=\int_{Z(\mathbb{A}) G_{0}(F) \backslash G_{0}(\mathbb{A})} \varphi(g) E\left(g, s, f_{s}\right) d g .
$$

Since $\omega_{\pi}=1$, this is well-defined. It is absolutely convergent by the moderate growth of Eisenstein series and the rapid decay of cusp forms (modulo the center). In order to understand this integral, we need a few auxiliary results.

Remark 2.1.3. We don't use the full center $Z_{0}$ of $G_{0}$ in the integral, but it is easy to check that

$$
Z(\mathbb{A}) Z_{0}(F) \backslash Z_{0}(\mathbb{A}) \simeq \mathbb{A}_{F}^{\times} E_{0}^{\times} \backslash \mathbb{A}_{E, 0}^{\times}
$$

with $E_{0}=\left\{\alpha \in E \mid \alpha^{2} \in F\right\}$. Now $\mathbb{A}_{F}^{\times} \simeq \mathbb{A}_{F}^{1} \times \mathbb{R}_{+}^{\times}$and similarly for $E$, and the natural embedding $\mathbb{A}_{F}^{\times} \hookrightarrow \mathbb{A}_{E}^{\times}$is the map

$$
\begin{gathered}
\mathbb{A}_{F}^{1} \times \mathbb{R}_{+}^{\times} \hookrightarrow \mathbb{A}_{E}^{1} \times \mathbb{R}_{+}^{\times}, \\
\left(\left(\alpha_{v}\right)_{v \in \Sigma_{F}}, r\right) \mapsto\left(\left(\alpha_{w}\right)_{w \in \Sigma_{E}}, r^{2}\right),
\end{gathered}
$$

where as usual $\alpha_{w}=\alpha_{v}$ whenever $w$ lies over $v$. So $\mathbb{A}_{F}^{\times} E_{0}^{\times} \backslash \mathbb{A}_{E, 0}^{\times} \simeq \mathbb{A}_{F}^{1} E_{0}^{\times} \backslash \mathbb{A}_{E, 0}^{1}$, and $E_{0}^{\times} \backslash \mathbb{A}_{E, 0}^{1} \hookrightarrow E^{\times} \backslash \mathbb{A}_{E}^{1}$, which is compact.

\subsection{Roots, Weights and Subgroups}

We will consider various subgroups of $\mathrm{GSp}_{4}(E)$ when analyzing our global integral. To clarify their structure, we start with root data. 
Lemma 2.2.1. Let $h=h\left(t_{1}, \ldots, t_{2 n}\right)$ denote the diagonal matrix in $\mathrm{GSp}_{2 n}$ with given entries from upper-left to lower-right, so that $t_{n+i}=\lambda t_{i}^{-1}$ for $1 \leq i \leq n$. Let $T_{2 n}$ be the subgroup of all such diagonal matrices, which is a maximal (split) torus in $\mathrm{GSp}_{2 n}$. Let $B_{2 n}$ be the Borel subgroup of $\mathrm{GSp}_{2 n}$ with Levi factor $T_{2 n}$ and unipotent radical equal to

$N^{B_{2 n}}=\left\{\left(\begin{array}{cc}A & \\ & \lambda^{t} A^{-1}\end{array}\right)\left(\begin{array}{cc}I_{n} & B \\ & I_{n}\end{array}\right) \mid A \in \mathrm{GL}_{n}\right.$ upper triangular unipotent, $\left.B \in \mathrm{Sym}_{n}\right\}$,

where "unipotent" denotes matrices with all diagonal entries equal to 1. Define characters $\epsilon_{i}$ of $T_{2 n}$ by

$$
\epsilon_{i}(h)=h^{\epsilon_{i}} \stackrel{\text { def }}{=} t_{i}, \quad 1 \leq i \leq 2 n .
$$

The based root system of $\left(\mathrm{GSp}_{2 n}, B_{2 n}, T_{2 n}\right)$ is $\left(X^{*}\left(T_{4}\right), \Phi, \Delta\right)$, where

$$
\begin{gathered}
X^{*}\left(T_{2 n}\right)=\bigoplus_{i=1}^{2 n} \mathbb{Z} \epsilon_{i} /\left\langle\epsilon_{i}+\epsilon_{n+i}-\epsilon_{i+1}-\epsilon_{n+i+1}: 1 \leq i<n\right\rangle \\
\Phi=\left\{ \pm\left(\epsilon_{i}-\epsilon_{j}\right), \pm\left(\epsilon_{i}-\epsilon_{j+n}\right): 1 \leq i \leq j \leq n\right\}-\{0\} \\
\Phi^{+}=\left\{\epsilon_{i}-\epsilon_{j}, \epsilon_{i}-\epsilon_{j+n}: 1 \leq i \leq j \leq n\right\}-\{0\} \\
\Delta=\left\{\alpha_{1}, \ldots, \alpha_{n}\right\} \\
\alpha_{i}=\epsilon_{i}-\epsilon_{i+1}, \quad 1 \leq i \leq n-1, \\
\alpha_{n}=\epsilon_{n}-\epsilon_{2 n} .
\end{gathered}
$$

Let $P_{4} \subseteq \mathrm{GSp}_{4}$ be the Klingen parabolic subgroup

$$
P_{4}=\left\{g=\left(\begin{array}{ccc}
* & * & * \\
* & * \\
* & * \\
* & * & *
\end{array}\right) \in \mathrm{GSp}_{4}\right\} .
$$


It has Levi subgroup isomorphic to $\mathrm{GL}_{2} \times \mathrm{GL}_{1}$. We will consider two " $F$-forms" $Q(F), Q^{\prime}(F)$ of $P_{4}(E)$, given as subgroups of $\mathrm{GSp}_{4}(E)$ by

$$
Q(F)=\left\{\left(\begin{array}{cccc}
e & x & w \theta & s^{\prime} \theta \\
a & s & s \theta \\
c \theta & e^{\prime} \\
c \theta & x^{\prime} & d
\end{array}\right) \mid a, \ldots, x^{\prime} \in F, a d-b c \theta^{2}=e e^{\prime}=\lambda(g)\right\}
$$

and

$$
\begin{aligned}
Q^{\prime}(F)=\left\{\left(\begin{array}{ccc}
e x(1+\theta) & w(1+\theta) & s^{\prime} \\
a & s & b(1-\theta) \\
c(1+\theta) & x^{\prime}(1+\theta) & d
\end{array}\right) \mid a, \ldots, x^{\prime} \in F,\right. & \left.a d-b c\left(1-\theta^{2}\right)=e e^{\prime}=\lambda(g)\right\} ;
\end{aligned}
$$

these are intersections of (maximal) parabolic subgroups of $\operatorname{GSp}_{8}(F)$ with $G_{0}(F)$. $Q$ and $Q^{\prime}$ should be thought of as analogues of the subgroups

$$
\begin{aligned}
P_{4}{ }^{\Delta} & =\left\{(g, g): g \in P_{4}\right\} \subseteq \mathrm{GSp}_{4} \times \mathrm{GSp}_{4}, \\
P_{4}{ }^{1} & =\left\{(g, 1): g \in P_{4}\right\} \subseteq \mathrm{GSp}_{4} \times \mathrm{GSp}_{4}
\end{aligned}
$$

under the usual doubling map $\left(\operatorname{GSp}_{4}(F) \times \operatorname{GSp}_{4}(F)\right)^{\circ} \hookrightarrow \operatorname{GSp}_{8}(F)$. They are "complementary" in the sense that if $Y(E)$ is a root subgroup of $P_{4}(E)$, then $(Y(E) \cap$ $Q(F)) \cdot\left(Y(E) \cap Q^{\prime}(F)\right)=Y(E)$. Finally, define $\gamma_{0} \in \mathrm{GSp}_{8}(F)$ by

$$
\gamma_{0}=\left(\begin{array}{ccccccc}
1 & & & 0 & 0 & 0 & 0 \\
& 0 & 0 & & 0 & 0 \\
& & 0 & & 0 & 1 \\
0 & & & 0 & 1 & 0 \\
& 0 & 0 & 0 & -1 & 0 & 1 \\
& -1 & 0 & & 0 & 0
\end{array}\right) \text {; }
$$

then $\gamma_{0}$ represents an element of the Weyl group of $\mathrm{GSp}_{8}(F)$ of order 3 .

For parabolic subgroups $P=P_{8}, P_{4}, Q, Q^{\prime}$ above, denote their respective Levi subgroups by $M^{P}$, their maximal unipotent subgroups by $N^{P}$ and their unipotent radicals by $U^{P}$. (So, for example, $N^{P_{4}}=N^{B_{4}}=U^{B_{4}}$ in the notation of Lemma 2.2.1.) 
Similarly the opposite unipotent subgroups will be $\bar{N}^{P}, \bar{U}^{P}$. The unipotent radicals $U^{P}$ for $P=P_{4}, Q, Q^{\prime}$ are Heisenberg groups, and we denote their centers by $X_{2}^{P}$; in fact, if $X_{2}$ denotes the root subgroup of $\mathrm{GSp}_{4}$ corresponding to the highest root $2 \alpha_{1}+\alpha_{2}$, then $X_{2}^{P}=X_{2} \cap P$. Moreover, $X_{2}^{P}$ is normalized by $P$ :

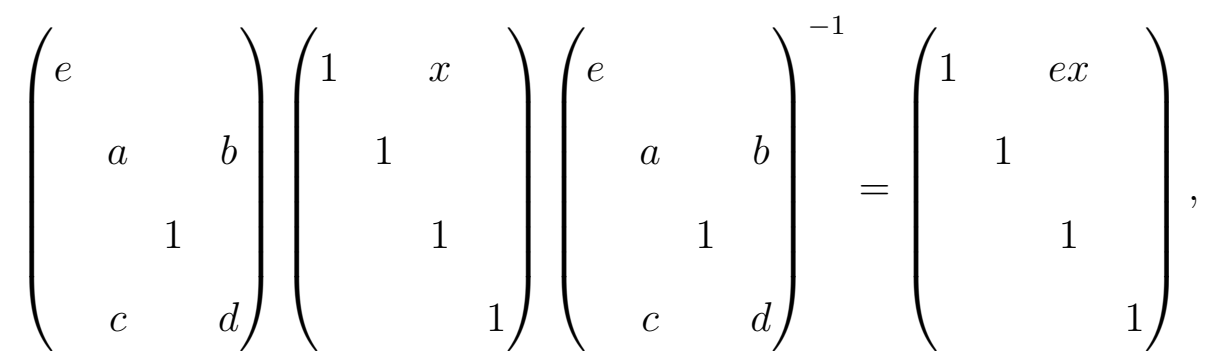

where $e=a d-b c$. One can check directly that

$$
Q(F) X_{2}^{Q^{\prime}}(F)=G_{0}(F) \cap \gamma_{0}^{-1} P_{8}(F) \gamma_{0}
$$

which we will need for the proof of Prop. 2.3.1. For convenience we often denote by $e(p)$ and $e^{\prime}(p)$ the $(1,1)$ - and $(3,3)$-entries of an element of $P_{4}, Q$ or $Q^{\prime}$, so that $e(p) e^{\prime}(p)=\lambda(p)$. We will also frequently make use (without mention) of the fact that for $P=P_{4}, Q, Q^{\prime}$, we can decompose $M^{P}$ as a commuting product of subgroups

$$
M^{P}=Z \cdot \widetilde{M}^{P}
$$

where

$$
\widetilde{M}^{P}=\left\{m \in M^{P} \mid e^{\prime}(m)=1\right\}
$$

Lastly we note two facts which will be needed for the unramified calculation.

Proposition 2.2.2 ([2], I.2.2(5)). The complex dual group of $\mathrm{GSp}_{4}$ is $\mathrm{GSp}_{4}(\mathbb{C})$. 
Proposition 2.2.3. Every finite-dimensional representation of $\mathrm{GSp}_{4}(\mathbb{C})$ is of the form $\lambda^{s} \otimes \tau_{(m, n)}$, where $\lambda$ is the similitude factor, $s \in \mathbb{C}$, and $\tau_{(m, n)}$ is the (finitedimensional) representation of $\mathrm{Sp}_{4}(\mathbb{C})$ of highest weight $(m, n)=m \omega_{1}+n\left(\omega_{1}+\omega_{2}\right)$.

\subsection{Orbit Decomposition}

We need to know the orbits of $G_{0}(F)$ on $X=P_{8}(F) \backslash \operatorname{GSp}_{8}(F)$. We may think of the latter as the variety of isotropic $F$-subspaces $L$ which are 3 -dimensional in $W=\operatorname{Res}_{E / F} V$. For such an isotropic $F$-subspace $L$, we can consider the number of independent $E$-lines contained in $L$. Equivalently, we may determine the dimension of $E \cdot L$, the $E$-subspace of $V$ generated by $L$. Let $d(L)=\operatorname{dim}_{E}(E \cdot L)$.

Proposition 2.3.1. $d(L)$ is a complete invariant for $G_{0}(F)$-orbits in $X$, and there are two such orbits, corresponding to $d(L)=2$ and $d(L)=3$. The former is negligible in the sense of [35] and the latter has stabilizer $Q(F) X_{2}^{Q^{\prime}}(F)$ (up to conjugacy).

Proof. Let $X^{(i)}$ be the collection of $L \in X$ with $d(L)=i$, so that $X$ is a union of the sets $X^{(i)}, i=1,2,3$. Since $E / F$ is quadratic, any $L \in X^{(1)}$ would have $F$-dimension 2 , so $X^{(1)}$ is empty.

Set $L_{1}=F \theta e_{2}+F e_{1}^{\prime}+F e_{2}^{\prime} \in X^{(3)}$. For $L^{\prime} \in X^{(3)}$, we may write $L^{\prime}=F v_{1}+F v_{2}+F v_{3}$, where $\left\langle v_{1}, v_{2}\right\rangle=\theta$ and $\left\langle v_{3}, L^{\prime}\right\rangle=0$. To see this, take any basis $\left\{v_{1}, v_{2}, v_{3}\right\}$ of $L^{\prime}$. Then $\left\langle\left\langle v_{i}, v_{j}\right\rangle\right\rangle=0$ for $1 \leq i, j \leq 3$. But $\operatorname{dim}_{E} V=4$, so $E \cdot L^{\prime}$ cannot be isotropic. Hence $\left\langle v_{i}, v_{j}\right\rangle \neq 0$ for some $i, j$; we may assume $i=1, j=2$. Since $\left\langle\left\langle v_{1}, v_{2}\right\rangle\right\rangle=0$, $\left\langle v_{1}, v_{2}\right\rangle \in F^{\times} \cdot \theta$. By scaling $v_{2}$, we can assume $\left\langle v_{1}, v_{2}\right\rangle=\theta$. Similarly, $\left\langle v_{1}, v_{3}\right\rangle=a \theta$ 
and $\left\langle v_{2}, v_{3}\right\rangle=b \theta$, for $a, b \in F$. Replacing $v_{3}$ by $v_{3}-a v_{2}+b v_{1}$ now gives what we want.

Now define a linear map $g_{1}: L^{\prime} \rightarrow L_{1}$ by

$$
\begin{aligned}
& v_{1} g_{1}=\theta e_{2}, \\
& v_{2} g_{1}=e_{2}^{\prime}, \\
& v_{3} g_{1}=e_{1}^{\prime} .
\end{aligned}
$$

By construction, $\left\langle v_{i} g_{1}, v_{j} g_{1}\right\rangle=\left\langle v_{i}, v_{j}\right\rangle$ for $1 \leq i, j \leq 3$. Now the Witt extension theorem shows $g_{1}$ extends to $g^{\prime} \in \operatorname{Sp}_{4}(E) \subseteq G_{0}$. Thus $G_{0}$ is transitive on $X^{(3)}$.

A similar argument applies to $X^{(2)}$. Let $L_{0}=F e_{1}^{\prime}+F \theta^{-1} e_{1}^{\prime}+F e_{2}^{\prime} \in X^{(2)}$. If $L^{\prime \prime} \in X^{(2)}$, there is a $0 \neq v \in L^{\prime \prime}$ such that $\theta v \in L^{\prime \prime}$ also. Set $v_{1}=v, v_{2}=\theta^{-1} v$ and extend to a basis $\left\{v_{1}, v_{2}, v_{3}\right\}$ of $L^{\prime \prime}$ over $F$. It is easy to see that $E \cdot L^{\prime \prime}$ is isotropic. If not, then $\left\langle v, v_{3}\right\rangle \neq 0$. Since $\left\langle\left\langle v, v_{3}\right\rangle\right\rangle=0$, we can assume (after scaling $\left.v_{3}\right)$ that $\left\langle v, v_{3}\right\rangle=\left\langle v_{1}, v_{3}\right\rangle=\theta$. Then $\left\langle v_{2}, v_{3}\right\rangle=\left\langle\theta^{-1} v, v_{3}\right\rangle=1$, so $\left\langle\left\langle v_{2}, v_{3}\right\rangle\right\rangle=1$, a contradiction.

Let $g_{0}: L^{\prime \prime} \rightarrow L_{0}$ be the linear map defined by

$$
\begin{aligned}
& v_{1} g_{0}=e_{1}^{\prime}, \\
& v_{2} g_{0}=\theta^{-1} e_{1}^{\prime}, \\
& v_{3} g_{0}=e_{2}^{\prime} .
\end{aligned}
$$

Then as before $g_{0}$ extends to a $g^{\prime \prime} \in \operatorname{Sp}_{4}(E) \subseteq G_{0}$, so $G_{0}$ is transitive on $X^{(2)}$.

Finally, it is clear that $X^{(2)}$ and $X^{(3)}$ are disjoint, since any $g \in G_{0}(F)$ is invertible and commutes with multiplication by elements of $E$. Moreover, as was noted above 
$L^{\prime} \in X^{(3)}$ implies that $E \cdot L$ is not (totally) isotropic, while $L^{\prime \prime} \in X^{(2)}$ implies $E \cdot L^{\prime \prime}$ is isotropic. Thus the orbits are exactly $X^{(2)}$ and $X^{(3)}$.

Now $L_{0} \in X^{(2)}$ has stabilizer $G_{0}(F) \cap P_{8}(F)$, which is (isomorphic to) the subgroup

$$
\left\{\left(\begin{array}{cccc}
* & * & * & * \\
& a_{22} & * & * \\
& & * & \\
& & * & d_{22}
\end{array}\right): a_{22}, d_{22} \in F\right\} \subseteq \operatorname{GSp}_{4}(E)
$$

which has unipotent radical $U^{B_{4}}(E)$, where $B_{4}$ is the Borel subgroup of $\mathrm{GSp}_{4}$. Hence $X^{(2)}$ is negligible.

For $X^{(3)}$, we can write $L_{1}=L_{0} \gamma_{0}$, where $\gamma_{0}$ is given by (2.9). Then the stabilizer of $L_{1} \in X^{(3)}$ is $G_{0} \cap \gamma_{0}^{-1} P_{8}(F) \gamma_{0}=Q(F) X_{2}^{Q^{\prime}}(F)$ as in (2.10).

\subsection{A Lemma on Cusp Forms}

For the unfolding of our integral, we need a way to introduce a "Whittaker expansion" for a cusp form $\varphi$ on $\operatorname{GSp}_{4}\left(\mathbb{A}_{E}\right)$. We will follow Jiang's lead [24, sec. 2.2.1].

For our purposes, let $\psi_{\mathrm{o}}$ be a fixed, non-degenerate additive character of $\mathbb{A}$, trivial on $F$, and $\psi: N^{B_{4}}\left(\mathbb{A}_{E}\right) \rightarrow \mathbb{C}^{\times}$be given by

$$
\psi\left(\begin{array}{cccc}
1 & x & * & * \\
1 & * & y \\
& 1 \\
& -x & 1
\end{array}\right)=\psi_{\circ}\left(\frac{1}{2} \operatorname{Tr}_{E / F}(\theta x+y)\right),
$$

so that $\left.\psi\right|_{N Q(\mathbb{A})} \equiv 1 . \psi$ is generic in the sense that it is not identically zero on either simple root subgroup of $\mathrm{GSp}_{4}$.

Recall that a cuspidal automorphic representation $\left(\pi, V_{\pi}\right)$ of $\operatorname{GSp}_{4}\left(\mathbb{A}_{E}\right)$ is said to be $\psi$-generic (or simply generic) if there is a nonzero linear functional $\Lambda: V_{\pi} \rightarrow \mathbb{C}$ 
satisfying $\Lambda(\pi(n) \varphi)=\psi(n) \Lambda(\varphi)$ for $n \in N^{B_{4}}\left(\mathbb{A}_{E}\right)$ and $\varphi \in V_{\pi}$. Note that if $\pi$ is $\psi$-generic, it will be $\psi^{\prime}$-generic for any other generic character $\psi^{\prime}$ of $N^{B_{4}}\left(\mathbb{A}_{E}\right)$; see $[26$, pp. 381-2]. For $\varphi \in V_{\pi}$, the Whittaker function associated to $\varphi$ and $\psi$ is defined to be

$$
W_{\varphi, \psi}(g)=\int_{N^{B_{4}}(E) \backslash N^{B_{4}}\left(\mathbb{A}_{E}\right)} \varphi(u g) \psi^{-1}(u) d u .
$$

$\pi$ is said to be globally $\psi$-generic if $W_{\varphi, \psi} \neq 0$ for some $\varphi \in V_{\pi}$. As above, such $\pi$ will be globally $\psi^{\prime}$-generic for every generic $\psi^{\prime}$, so we simply say $\pi$ is globally generic.

Consider the parabolic subgroup $P_{4}$. It has as a normal subgroup the root subgroup $X_{2}=X_{2}^{P_{4}}$, which is the center of $N^{P_{4}}$. Taking the quotient by this and the center $Z$ of $\mathrm{GSp}_{4}$, we have a natural projection onto the (upper triangular) mirabolic subgroup $P_{3}^{\prime}$ in $\mathrm{GL}_{3}$ :

$$
\begin{aligned}
& \rho: P_{4}\left(\mathbb{A}_{E}\right) \rightarrow P_{3}^{\prime}\left(\mathbb{A}_{E}\right),
\end{aligned}
$$

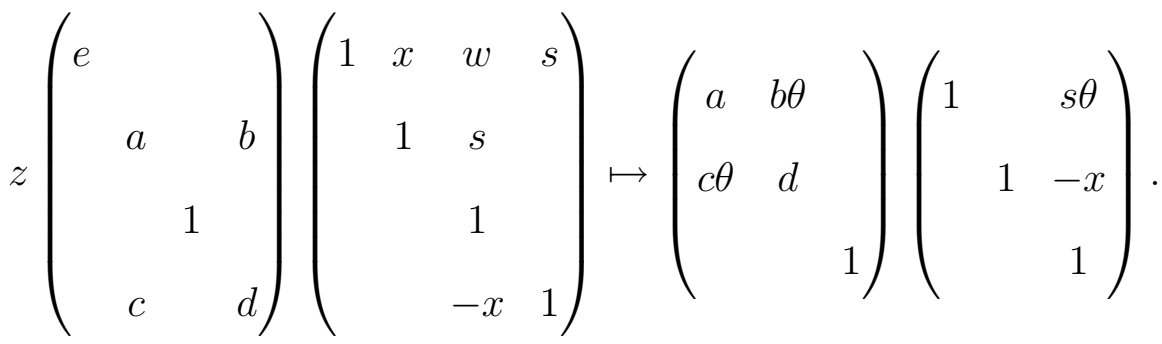

Note that we have decomposed the Levi of $P_{4}$ as $Z$ times a subgroup isomorphic to $\mathrm{GL}_{2}$ and as above, $e=a d-b c$.

Lemma 2.4.1. (i). $\rho$ is surjective with kernel $X_{2} \cdot Z$, where $Z$ is the center of $\mathrm{GSp}_{4}$.

(ii). $\rho^{-1}\left(P_{3}^{\prime}(F)\right)=Z(E) X_{2}(E) \cdot Q(F)$ and $\rho^{-1}\left(N_{3}(F)\right)=Z(E) X_{2}(E) \cdot N^{Q}(F)$.

Proof. Follows directly from the definitions of $\rho, Q$ and $N^{Q}$. 
Following Jiang, for a cusp form $\varphi$ of $\pi$ we introduce the function

$$
\varphi^{*}(g)=\int_{X_{2}(E) \backslash X_{2}\left(\mathbb{A}_{E}\right)} \varphi(x g) d x .
$$

Remark 2.4.2. (i). The idea to use such an integration comes from [11] and is also found in [10].

(ii). If $\pi$ is generic and $\varphi^{*} \equiv 0$ for all $\varphi \in V_{\pi}, \pi$ is called "hypercuspidal" by Gelbart and Piatetski-Shapiro. By (2.16) and (2.21) below, we have

$$
\varphi^{*}(g)=\sum_{\tilde{\alpha} \in Z(E) N^{P_{4}}(E) \backslash P_{4}(E)} W_{\varphi, \psi}(\tilde{\alpha} g)
$$

so if $\varphi^{*} \equiv 0$ for all $\varphi \in V_{\pi}$, then $W_{\varphi, \psi} \equiv 0$ for all $\varphi$ and $\psi$, which implies $\pi$ is not globally generic. So a "hypercuspidal" representation in this context is one which is generic but not globally generic. It is not known whether such representations exist (cf. [26], p. 383).

When $\varphi^{*}$ is restricted to $P_{4}\left(\mathbb{A}_{E}\right)$, it has a natural push-forward under $\rho$, namely

$$
\left\{\begin{array}{c}
\phi: P_{3}^{\prime}\left(\mathbb{A}_{E}\right) \rightarrow \mathbb{C} \\
\phi(r)=\varphi^{*}\left(\rho^{-1}(r) g\right)
\end{array}\right.
$$

for $g \in \operatorname{GSp}_{4}\left(\mathbb{A}_{E}\right)$. Since $\pi$ has trivial central character and by the previous lemma, this is a well-defined function on $P_{3}^{\prime}\left(\mathbb{A}_{E}\right)$ which is clearly left-invariant under $P_{3}^{\prime}(E)$ and is in fact a cuspidal automorphic form on $P_{3}^{\prime}\left(\mathbb{A}_{E}\right) \subseteq \mathrm{GL}_{3}\left(\mathbb{A}_{E}\right)$. We will see below that $\phi$ is generic if and only if $\varphi$ is. We give an expansion for $\phi$ in terms of its Whittaker functions, following Shalika [38]; see [36]. The argument is due to Jacquet-Langlands [15] for $\mathrm{GL}_{2}$ and independently to Piatetski-Shapiro ([37]; see [36, 
p. 210]) and Shalika for $\mathrm{GL}_{n}, n \geq 3$. First, define a character $\psi^{\prime}$ on the maximal unipotent subgroup $N_{3}\left(\mathbb{A}_{E}\right) \subseteq \mathrm{GL}_{3}\left(\mathbb{A}_{E}\right)$ by

$$
\psi^{\prime}\left(\begin{array}{rrr}
1 & y & z \\
& 1 & x \\
& & 1
\end{array}\right)=\psi_{\circ}\left(\frac{1}{2} \operatorname{Tr}_{E / F}(\theta(x+y))\right)
$$

so that for $n \in N^{P_{4}}\left(\mathbb{A}_{E}\right)$ we have $\psi(n)=\psi^{\prime}(\rho(n))$, and $\left.\psi^{\prime}\right|_{N_{3}\left(\mathbb{A}_{F}\right)}=\left.\psi\right|_{N^{Q}\left(\mathbb{A}_{F}\right)} \equiv 1$. Then the Whittaker expansion for $\phi$ is

$$
\phi(r)=\sum_{\alpha \in N_{3}(E) \backslash P_{3}^{\prime}(E)} W_{\phi, \psi^{\prime}}(\alpha r),
$$

where

$$
W_{\phi, \psi^{\prime}}(r)=\int_{N_{3}(E) \backslash N_{3}\left(\mathbb{A}_{E}\right)} \phi(n r) \psi^{\prime-1}(n) d n .
$$

We want to modify this expansion to include only the $F$-rational terms.

Lemma 2.4.3. Let $\phi$ be a cusp form on $\mathrm{GL}_{n}\left(\mathbb{A}_{E}\right)$ or on the mirabolic subgroup $P_{n}^{\prime}\left(\mathbb{A}_{E}\right)$ and $\chi$ a nondegenerate character on the maximal unipotent subgroup $N_{n}\left(\mathbb{A}_{E}\right)$ of $\mathrm{GL}_{n}\left(\mathbb{A}_{E}\right)$ which is trivial on $N_{n}(E)$ and $N_{n}\left(\mathbb{A}_{F}\right)$. Then

$$
\int_{N_{n}(F) \backslash N_{n}\left(\mathbb{A}_{F}\right)} \phi(n g) d n=\sum_{\alpha \in N_{n-1}(F) \backslash \mathrm{GL}_{n-1}(F)} W_{\phi, \chi}\left(\left(\begin{array}{ll}
\alpha & \\
& 1
\end{array}\right) g\right) .
$$

Remark 2.4.4. This result is also used by Flicker [8] to unfold his global integral into an Euler product.

Proof of Lemma 2.4.3. This is the argument given in [38] with a minor modification; we give it here for completeness. The case $n=1$ is trivial, so assume $n \geq 2$. Let 
$P_{n}^{\prime}$ be the mirabolic (upper triangular) subgroup of $\mathrm{GL}_{n}$ and let $V_{n}$ be its unipotent radical,

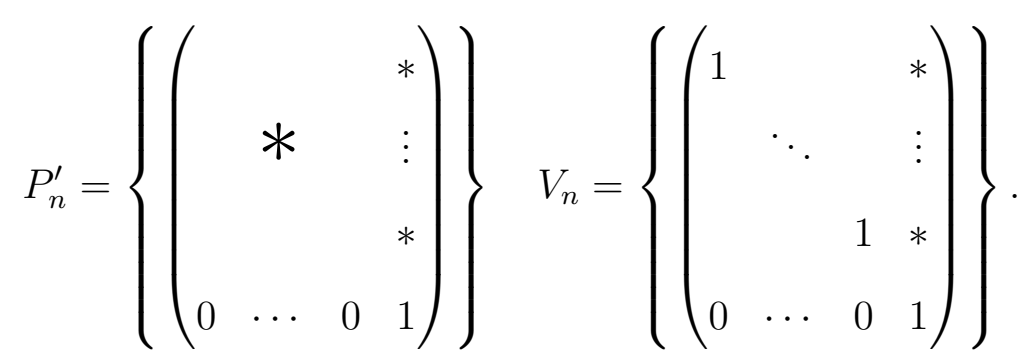

Define, for $x \in \mathrm{GL}_{n-1}\left(\mathbb{A}_{E}\right)$,

$$
F_{g}(x)=\int_{V_{n}(E) \backslash V_{n}\left(\mathbb{A}_{E}\right)} \phi\left(v\left(\begin{array}{ll}
x & \\
& 1
\end{array}\right) g\right) \chi^{-1}(v) d v
$$

so that for $\alpha \in \mathrm{GL}_{n-1}(E)$

$$
\begin{aligned}
F_{g}(\alpha) & =\int_{V_{n}(E) \backslash V_{n}\left(\mathbb{A}_{E}\right)} \phi\left(v\left(\begin{array}{ll}
\alpha & \\
& 1
\end{array}\right) g\right) \chi^{-1}(v) d v \\
& =\int_{V_{n}(E) \backslash V_{n}\left(\mathbb{A}_{E}\right)} \phi(v g) \chi^{-1}\left(\left(\begin{array}{ll}
\alpha & \\
& \\
& 1
\end{array}\right) v\left(\begin{array}{ll}
\alpha & \\
& \\
&
\end{array}\right)^{-1}\right) d v \\
& =\int_{V_{n}(E) \backslash V_{n}\left(\mathbb{A}_{E}\right)} \phi(v g) \chi_{\alpha}^{-1}(v) d v
\end{aligned}
$$

so by basic Fourier analysis we get

$$
\phi(v g)=\sum_{\alpha \in P_{n-1}^{\prime}(E) \backslash \mathrm{GL}_{n-1}(E)} F_{g}(\alpha) \chi_{\alpha}(v) .
$$

Now take $v \in V_{n}\left(\mathbb{A}_{F}\right)$ and integrate:

$$
\int_{V_{n}(F) \backslash V_{n}\left(\mathbb{A}_{F}\right)} \phi(v g) d v=\sum_{\alpha \in P_{n-1}^{\prime}(E) \backslash G L_{n-1}(E)} F_{g}(\alpha) \int_{V_{n}(F) \backslash V_{n}\left(\mathbb{A}_{F}\right)} \chi_{\alpha}(v) d v
$$


Now the integral on the right is nonzero if and only if $\left.\chi_{\alpha}\right|_{\mathbb{A}_{F}} \equiv 1$. But by definition of $\chi_{\alpha}$, this holds if and only if the last row of $\alpha$ has all entries in $F$.

Now look at the Bruhat-type decomposition in $\mathrm{GL}_{m}$,

$$
\mathrm{GL}_{m}=\coprod_{w \in W_{P_{m}^{\prime}} \backslash W_{m}} P_{m}^{\prime} w B_{m},
$$

where $B_{m}$ is the upper triangular Borel subgroup of $\mathrm{GL}_{m}, W_{m}$ is the Weyl group of $\mathrm{GL}_{m}$ and $W_{P_{m}^{\prime}}$ is the Weyl group for $P_{m}^{\prime}$. The quotient $W_{P_{m}^{\prime}} \backslash W_{m}$ has order $m$ and is represented by elements $w_{j}, 1 \leq j \leq m$, where left multiplication by $w_{j}$ exchanges the $j$-th and $m$-th rows. Let $\mathcal{O}_{j}(E)=P_{m}^{\prime}(E) \backslash P_{m}^{\prime}(E) w_{j} B_{m}(E)$, so we can choose as a representative of $\alpha \in \mathcal{O}_{j}(E)$ an element of the form

$$
\left(\begin{array}{llll}
1 & & & \\
& \ddots & & \\
& & 1 & \\
& & & t
\end{array}\right) w_{j} u
$$

for $t \in E^{\times}$and $u \in N_{m}(E)$, the unipotent radical of $B_{m}(E)$. But it is easy to see that we can decompose $u=u^{\prime} u^{\prime \prime}$, where $u^{\prime} \in N_{m}(E) \cap w_{j}^{-1} P_{m}^{\prime}(E) w_{j}$ and $u^{\prime \prime}$ has the 
form

$$
\begin{gathered}
u^{\prime \prime}=\left(\begin{array}{cc}
I_{j} & B \\
& I_{m-j}
\end{array}\right), \\
I_{j}=\text { the } j \times j \text { identity matrix, } \\
B=\left(\begin{array}{ccc}
0 & \ldots & 0 \\
\vdots & & \vdots \\
0 & \ldots & 0 \\
b_{1} & \ldots & b_{m-j}
\end{array}\right) \in \operatorname{Mat}_{j \times(m-j)}(E) .
\end{gathered}
$$

So $\alpha \in \mathcal{O}_{j}(E)$ has a representative with entries 1 and 0 above the last row, and last row entries $\left(0, \ldots, 0, t, t b_{1}, \ldots, t b_{m-j}\right)$. But the $\alpha$-summand in the right-hand side of (2.18) is nonzero only if the last row of $\alpha$ is in $F$. So we may assume that $\alpha$ itself is in $\mathcal{O}_{j}(F)$. That is, the sum in $(2.18)$ may be taken over $\alpha \in P_{n-1}^{\prime}(F) \backslash \mathrm{GL}_{n-1}(F)$. Continuing inductively now gives the result.

Applying the lemma to our cusp form $\phi$ gives

$$
\int_{N_{3}(F) \backslash N_{3}\left(\mathbb{A}_{F}\right)} \phi(n r) d n=\sum_{\alpha \in N_{3}(F) \backslash P_{3}^{\prime}(F)} W_{\phi, \psi^{\prime}}(\alpha r),
$$

where we have used the standard fact $N_{n-1}(F) \backslash \mathrm{GL}_{n-1}(F)=N_{n}(F) \backslash P_{n}^{\prime}(F)$. Using the definition of $\phi$ from (2.14) and taking $r=1$, the integral becomes

$$
\begin{aligned}
\int_{N_{3}(F) \backslash N_{3}\left(\mathbb{A}_{F}\right)} \phi(n) d n & =\int_{N_{3}(F) \backslash N_{3}\left(\mathbb{A}_{F}\right)} \varphi^{*}\left(\rho^{-1}(n) g\right) d n \\
& =\int_{N^{Q}(F) X_{2}^{Q}\left(\mathbb{A}_{F}\right) \backslash N^{Q}\left(\mathbb{A}_{F}\right)} \varphi^{*}(u g) d u,
\end{aligned}
$$


whereas the Whittaker function $W_{\phi, \psi^{\prime}}$ is just

$$
\begin{aligned}
& W_{\phi, \psi^{\prime}}(\alpha)=\int_{N_{3}(E) \backslash N_{3}\left(\mathbb{A}_{E}\right)} \phi(n \alpha) \psi^{\prime-1}(n) d n \\
& =\int_{N_{3}(E) \backslash N_{3}\left(\mathbb{A}_{E}\right)} \int_{X_{2}(E) \backslash X_{2}\left(\mathbb{A}_{E}\right)} \varphi\left(x \rho^{-1}(n \alpha) g\right) \psi^{\prime-1}(n) d x d n \\
& =\int_{N^{P_{4}}(E) \backslash N^{P_{4}}\left(\mathbb{A}_{E}\right)} \varphi(u \tilde{\alpha} g) \psi^{-1}(u) d u=W_{\varphi, \psi}(\tilde{\alpha} g),
\end{aligned}
$$

where $\tilde{\alpha}$ is any preimage of $\alpha$ under $\rho$. Using these, (2.19) becomes

$$
\int_{N^{Q}(F) X_{2}^{Q}\left(\mathbb{A}_{F}\right) \backslash N^{Q}\left(\mathbb{A}_{F}\right)} \varphi^{*}(u g) d u=\sum_{\tilde{\alpha} \in Z(F) N^{Q}(F) \backslash Q(F)} W_{\varphi, \psi}(\tilde{\alpha} g) .
$$

\subsection{The Global Integral}

Proposition 2.5.1 (Main identity).

$$
I\left(s, \varphi, f_{s}\right)=\int_{\mathcal{D}(\mathbb{A})} f_{s}\left(\gamma_{0} g\right) W_{\varphi, \psi}(g) d g
$$

where $I\left(s, \varphi, f_{s}\right)$ is given by $(2.5)$ and $\mathcal{D}(\mathbb{A})=Z(\mathbb{A}) X_{2}^{Q^{\prime}}(\mathbb{A}) N^{Q}(\mathbb{A}) \backslash G_{0}(\mathbb{A})$.

Proof.

$$
\begin{aligned}
I\left(s, \varphi, f_{s}\right) & =\int_{Z(\mathbb{A}) G_{0}(F) \backslash G_{0}(\mathbb{A})} \varphi(g) E\left(g, s, f_{s}\right) d g \\
& =\int_{Z(\mathbb{A}) G_{0}(F) \backslash G_{0}(\mathbb{A})} \varphi(g) \sum_{\gamma \in P_{8}(F) \backslash \operatorname{GSp}_{8}(F)} f_{s}(\gamma g) d g \\
& =\sum_{\gamma \in P_{8}(F) \backslash \mathrm{GSp}_{8}(F) / G_{0}(F)} \int_{Z(\mathbb{A}) G_{0}^{\gamma}(F) \backslash G_{0}(\mathbb{A})} \varphi(g) f_{s}(\gamma g) d g .
\end{aligned}
$$

Since we have "unfolded" the Eisenstein series, each integral on the right is now convergent for $\operatorname{Re}(s)$ sufficiently large. By Prop. 2.3.1, the sum is over two orbits, 
and one is negligible. The non-negligible orbit is represented by $\gamma_{0}$ as in (2.9), and the stabilizer of this orbit is $G_{0}{ }^{\gamma_{0}}(F)=Q(F) X_{2}^{Q}(F)$ by (2.10). Then the integral is just

$$
I\left(s, \varphi, f_{s}\right)=\int_{Z(\mathbb{A}) Q(F) X_{2}^{Q^{\prime}}(F) \backslash G_{0}(\mathbb{A})} \varphi(g) f_{s}\left(\gamma_{0} g\right) d g .
$$

Now we follow Jiang's lead in projecting our cusp form onto the mirabolic in $\mathrm{GL}_{3}$ :

$$
\begin{aligned}
I\left(s, \varphi, f_{s}\right)=\int_{Z(\mathbb{A}) Q(F) X_{2}\left(\mathbb{A}_{E}\right) \backslash G_{0}(\mathbb{A})} f_{s}\left(\gamma_{0} g\right) \int_{X_{2}(E) \backslash X_{2}\left(\mathbb{A}_{E}\right)} \varphi(x g) d x d g \\
=\int_{Z(\mathbb{A}) X_{2}\left(\mathbb{A}_{E}\right) Q(F) \backslash G_{0}(\mathbb{A})} f_{s}\left(\gamma_{0} g\right) \varphi^{*}(g) d g \\
=\int_{Z(\mathbb{A}) X_{2}\left(\mathbb{A}_{E}\right) N^{Q}(\mathbb{A}) Q(F) \backslash G_{0}(\mathbb{A})} f_{s}\left(\gamma_{0} g\right) \int_{N^{Q}(F) X_{2}^{Q}(\mathbb{A}) \backslash N^{Q}(\mathbb{A})} \varphi^{*}(u g) d u d g \\
=\int_{Z(\mathbb{A}) X_{2}\left(\mathbb{A}_{E}\right) N^{Q}(\mathbb{A}) Q(F) \backslash G_{0}(\mathbb{A})} f_{s}\left(\gamma_{0} g\right) W_{\varphi \in Z(F) N^{Q}(F) \backslash Q(F)}(\alpha g) d g \\
=\int_{Z(\mathbb{A}) X_{2}\left(\mathbb{A}_{E}\right) N^{Q}(\mathbb{A}) \backslash G_{0}(\mathbb{A})} f_{s}\left(\gamma_{0} g\right) W_{\varphi, \psi}(g) d g
\end{aligned}
$$

since $\gamma_{0} \alpha \gamma_{0}^{-1} \in P_{8}(F)$. Finally, since $X_{2}\left(\mathbb{A}_{E}\right)=X_{2}^{Q}(\mathbb{A}) X_{2}^{Q^{\prime}}(\mathbb{A})$ and $X_{2}^{Q}(\mathbb{A}) \subseteq N^{Q}(\mathbb{A})$, this last integral is equal to

$$
\int_{Z(\mathbb{A}) X_{2}^{Q^{\prime}}(\mathbb{A}) N^{Q}(\mathbb{A}) \backslash G_{0}(\mathbb{A})} f_{s}\left(\gamma_{0} g\right) W_{\varphi, \psi}(g) d g,
$$

as desired.

Fix a tensor product decomposition $\pi \simeq \otimes_{v}^{\prime} \pi_{v}$. By the local uniqueness of Whittaker models, if we choose the section $f_{s}$ to be factorizable and $\varphi \simeq \otimes_{v} \xi_{v}$ to correspond to a pure tensor under this isomorphism, the right-hand integral in (2.23) factors as

$$
\begin{aligned}
\int_{\mathcal{D}(\mathbb{A})} f_{s}\left(\gamma_{0} g\right) W_{\varphi, \psi}(g) d g & =\prod_{v \in \Sigma_{F}} \int_{\mathcal{D}\left(F_{v}\right)} f_{s, v}\left(\gamma_{0} g_{v}\right) W_{\xi_{v}, \psi_{v}}\left(g_{v}\right) d g_{v} \\
& =\prod_{v \in \Sigma_{F}} \Psi_{v}\left(s, W_{\xi_{v}, \psi_{v}}, f_{s, v}\right),
\end{aligned}
$$


with $\Sigma_{F}$ the set of places of $F$.

Proposition 2.5.2. The infinite product on the right converges for $\operatorname{Re}(s)>>0$.

Proof. By Thm. 3.1.14 and Thm. 3.2.5, we have

$$
\Psi_{v}^{*}\left(s, W_{v}, f_{s, v}\right) \stackrel{\text { def }}{=} d_{G, v}(s) \Psi_{v}\left(s, W_{v}, f_{s, v}\right)=L_{v}\left(s, \pi_{v}, \rho_{\mathrm{TT}}\right)
$$

for almost all (i.e., all but finitely many) places $v$ of $F$. Let $S \subseteq \Sigma_{F}$ be the finite set of places $v$ for which this equality does not hold. (See the next section for a more explicit definition of $S$.) Then we can write

$$
\begin{aligned}
\prod_{v \in \Sigma_{F}} \Psi_{v}^{*}\left(s, W_{v}, f_{s, v}\right) & =\prod_{v \in S} \Psi_{v}^{*}\left(s, W_{v}, f_{s, v}\right) \cdot \prod_{v \notin S} \Psi_{v}^{*}\left(s, W_{v}, f_{s, v}\right) \\
& =\prod_{v \in S} \Psi_{v}^{*}\left(s, W_{v}, f_{s, v}\right) \cdot \prod_{v \notin S} L_{v}\left(s, \pi_{v}, \rho_{\mathrm{TT}}\right) .
\end{aligned}
$$

The product over $v \notin S$ is absolutely convergent for $\operatorname{Re}(s)>>0$ by [32, p. 45]. Each integral $\Psi_{v}^{*}\left(s, W_{v}, f_{s, v}\right)$ is absolutely convergent for $\operatorname{Re}(s)>>0$ by Prop. 4.1.7 and Prop. 4.1.8. Since $S$ is a finite set, the product over $v \in S$ is also absolutely convergent for $\operatorname{Re}(s)>>0$.

We will show that at almost all places this local integral computes the local twisted tensor $L$-factor, after suitable normalization of the Eisenstein series. Thus our (normalized) global integral represents the partial twisted tensor $L$-function. 


\section{CHAPTER 3 UNRAMIFIED COMPUTATION}

Let $S \subset \Sigma_{F}$ be a finite set of places of $F$ such that for $v \notin S, v$ is finite, unramified in $E$, of residue characteristic not 2, such that $\theta_{v}$ is a unit and such that $\pi_{v}$ and $\psi_{v}^{\circ}$ are also unramified. The place $v$ is either inert or split in $E$. If $v$ is split, we will show that the local integral agrees with Jiang's local unramified integral in [24]. We will also give a different proof that this integral calculates the local $L$-factor, based on branching laws. (Jiang's proof uses the Kostant-Rallis algorithm [29].) However, since the combinatorics in the split case are slightly more involved, we will do the inert case first.

\subsection{Inert Case}

Suppose $v$ is inert in $E$. As is customary, we omit the subscript $v$ henceforth. Fix a uniformizer $\varpi$ for $F$ (hence for $E$ ), let $\mathcal{O}=\mathcal{O}_{F}$ be the ring of integers of $F$ and $\mathfrak{p}=\mathfrak{p}_{F}$ be the maximal ideal in $\mathcal{O}$, and denote by $q=q_{F}$ the order of the residue field $\mathcal{O} / \mathfrak{p}$ of $F$, so $q_{E}=q_{F}^{2}$. Take $f_{s}^{\circ}$ and $W^{\circ}$ to be the spherical section and spherical Whittaker function, respectively, normalized so that $f_{s}^{\circ}(e)=W^{\circ}(e)=1$. This section will closely parallel section II.3.2 of [24]. 
We have the Iwasawa decomposition $\operatorname{GSp}_{4}(E)=N^{B_{4}}(E) T_{4}(E) K_{4}$, where $K_{4}=$ $\operatorname{GSp}_{4}\left(\mathcal{O}_{E}\right)$. By restricting similitudes to lie in $F$, this becomes

$$
G_{0}(F)=N_{0}(F) T_{0}(F) K_{0}
$$

where as sets $N_{0}(F)=N^{B_{4}}(E)$. Note that $T_{0}(F)$ is not split over $F$ ! Also, using the above notation we have $X_{2}^{Q^{\prime}}(F) N^{Q}(F) \backslash N_{0}(F) \simeq X_{2}^{Q^{\prime}}(F) \backslash N^{Q^{\prime}}(F)$. Using this decomposition, the local integral becomes

$$
\begin{aligned}
\Psi(s, & \left.W^{\circ}, f_{s}^{\circ}\right)=\int_{\mathcal{D}(F)} f_{s}^{\circ}\left(\gamma_{0} g\right) W^{\circ}(g) d g \\
= & \int_{K_{0} \cap Z(F) \backslash K_{0}} \int_{Z(F) \backslash T_{0}(F)} \\
& \cdot \int_{X_{2}^{Q^{\prime}}(F) N^{Q}(F) \backslash N_{0}(F)} f_{s}^{\circ}\left(\gamma_{0} n t k\right) W^{\circ}(n t k) \delta_{B_{0}}^{-1}(t) d n d t d k \\
= & \int_{Z(F) \backslash T_{0}(F)} W^{\circ}(t) \delta_{B_{0}}^{-1}(t) \int_{X_{2}^{Q^{\prime}}(F) \backslash N Q^{\prime}(F)} f_{s}^{\circ}\left(\gamma_{0} n t\right) \psi(n) d n d t,
\end{aligned}
$$

where $\delta_{B_{0}}$ is the modulus of the Borel subgroup $B_{0} \subseteq G_{0}$. It is easy to see that $\delta_{B_{0}}=\delta_{B_{4}}^{2}$ is the square of the modulus character for the Borel of $\mathrm{GSp}_{4}$.

We first evaluate the inner integral $I\left(t, f_{s}^{\circ}, \psi\right)$ by reducing it to a product of onevariable integrals, and then we will use the formula of Casselman-Shalika [5] to compute the (non-normalized) unramified integral.

\subsubsection{The Inner Integral $I\left(t, f_{s}^{\circ}, \psi\right)$}

Denote by

$$
h(a, b, c, d), \quad a, b, c, d \in E
$$


and

$$
h\left(a_{1}, a_{2}, a_{3}, a_{4}, a_{5}, a_{6}, a_{7}, a_{8}\right), \quad a_{1}, \ldots, a_{8} \in F
$$

the diagonal matrices in $\mathrm{GSp}_{4}(E)$ and $\mathrm{GSp}_{8}(F)$, respectively, with given entries from upper-left to lower-right. Set $K^{\prime}=K^{\prime}(F)=\operatorname{GSp}_{8}\left(\mathcal{O}_{F}\right)$. For a root $\alpha$, let $\chi_{\alpha}$ denote the corresponding root subgroup (so $t \cdot \chi_{\alpha}(x) \cdot t^{-1}=\chi_{\alpha}(\alpha(t) x)$ ). Define

$$
\begin{aligned}
& \chi(x, y, w, u, v)=\chi_{-\epsilon_{2}-\epsilon_{4}}(x) \chi_{-2 \epsilon_{3}}(y) \chi_{-\epsilon_{3}-\epsilon_{4}}(w) \chi_{-\epsilon_{1}-\epsilon_{4}}(u) \chi_{-\epsilon_{2}-\epsilon_{3}}(v)
\end{aligned}
$$

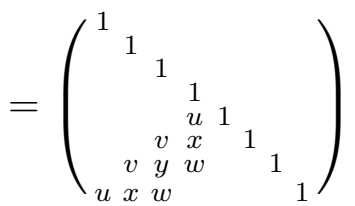

Set $\chi(x)=\chi(x, 0,0,0,0), \chi(x, y)=\chi(x, y, 0,0,0)$, etc. (Our $\chi(x, y, w, u, v)$ is denoted $\chi(w, y, x, u, v)$ in [24].)

Lemma 3.1.1. We have the following:

(i). $h\left(a_{1}, a_{2}, a_{3}, a_{4}, a_{5}, a_{6}, a_{7}, a_{8}\right)^{-1} \chi(x, y, w, u, v) h\left(a_{1}, a_{2}, a_{3}, a_{4}, a_{5}, a_{6}, a_{7}, a_{8}\right)$ $=\chi\left(a_{4} a_{6}^{-1} x, a_{3} a_{7}^{-1} y, a_{4} a_{7}^{-1} w, a_{4} a_{5}^{-1} u, a_{3} a_{6}^{-1} v\right)$;

(ii). $\delta_{P_{8}}\left(h\left(a_{1}, a_{2}, a_{3}, a_{4}, a_{5}, a_{6}, a_{7}, a_{8}\right)\right)=\left(\frac{a_{1}^{2} a_{2}^{2}}{a_{3} a_{7}^{3}}\right)^{3}=\left(\frac{a_{1}^{2} a_{3}^{2}}{a_{2} a_{6}^{3}}\right)^{3}=\left(\frac{a_{2}^{2} a_{3}^{2}}{a_{1} a_{5}^{3}}\right)^{3}$;

(iii). If $|v|>1, \chi_{-\epsilon_{2}-\epsilon_{3}}(v)=h\left(1, v^{-1}, v^{-1}, 1,1, v, v, 1\right) \chi_{\epsilon_{2}+\epsilon_{3}}(v) k_{v}$, for some $k_{v} \in K^{\prime}$;

(iv). $\chi(x, y, w, u) \chi_{\epsilon_{2}+\epsilon_{3}}(v)=p_{1}(x, y, w, v) \chi(x, y, w-v x y, u)$, where $p_{1}(x, y, w, v) \in$ $P_{8}(F)$ satisfies $\delta_{P_{8}}\left(p_{1}(x, y, w, v)\right)=1$;

(v). If $|u|>1, \chi_{-\epsilon_{1}-\epsilon_{4}}(u)=h\left(u^{-1}, 1,1, u^{-1}, u, 1,1, u\right) \chi_{\epsilon_{1}+\epsilon_{4}}(u) k_{u}$, for some $k_{u} \in K^{\prime}$; 
(vi). $\chi(x, y, w) \chi_{\epsilon_{1}+\epsilon_{4}}(u)=p_{2}(x, w, u) \chi(x, y, w)$, where $p_{2}(x, w, u) \in P_{8}(F)$ satisfies $\delta_{P_{8}}\left(p_{2}(x, w, u)\right)=1 ;$

(vii). If $|x|>1, \chi_{-\epsilon_{2}-\epsilon_{4}}(x)=h\left(1, x^{-1}, 1, x^{-1}, 1, x, 1, x\right) \chi_{\epsilon_{2}+\epsilon_{4}}(x) k_{x}$, for some $k_{x} \in K^{\prime}$;

(viii). If $|y|>1, \chi_{-2 \epsilon_{3}}(y)=h\left(1,1, y^{-1}, 1,1,1, y, 1\right) \chi_{2 \epsilon_{3}}(y) k_{y}$, for some $k_{y} \in K^{\prime}$.

(ix). $\chi(x, 0, w) \chi_{2 \epsilon_{3}}(y)=p_{3}(y, w) \chi(x, 0, w)$, where $p_{3}(y, w) \in P_{8}(F)$ satisfies $\delta_{P_{8}}\left(p_{3}(y, w)\right)=1$

(x). $\chi(x) \chi_{\epsilon_{3}+\epsilon_{4}}(w)=p_{4}(x, w) \chi(x)$, where $p_{4}(x, w) \in P_{8}(F)$ satisfies $\delta_{P_{8}}\left(p_{4}(x, w)\right)=$ 1.

Proof. All but the last two statements are found in [24]; all are standard computations.

Lemma 3.1.2. For $t=h\left(a, b, \lambda a^{-1}, \lambda b^{-1}\right)$ and $a=a_{1}+a_{2} \theta, b=b_{1}+b_{2} \theta$, write $\mathbb{N} a=\mathrm{N}_{E / F}(a)$ and similar for $\mathbb{N} b$. Then

$$
\begin{aligned}
I\left(t, f_{s}^{\circ}, \psi\right)= & \left|a_{1}\right|^{-s-1}\left|b_{1}\right|^{-2 s-2}|\mathbb{N} a|^{s+3}|\mathbb{N} b|^{s+2}|\lambda|^{-\frac{s+7}{2}} \\
& \cdot \int_{F^{3}} f_{s}^{\circ}\left(\chi\left(x, y, w,-\frac{a_{2} \theta^{2}}{a_{1}},-\frac{b_{2} \theta^{2}}{b_{1}}\right)\right) \psi_{\circ}\left(-\frac{a_{1} b_{1}}{\mathbb{N} b} x-\frac{b_{1}^{2}}{\lambda \theta^{2}} y\right) d x d y d w .
\end{aligned}
$$

Proof. By direct calculation, we have $\gamma_{0} n t=n^{\prime} p \bar{n} \gamma_{0}$, where $n^{\prime}, p \in P_{8}(F)$ and $n^{\prime}$ is unipotent, and $\bar{n} \in \bar{U}^{P}(F)$. Since $\gamma_{0} \in K_{0}(F)$ we will have $f_{s}^{\circ}\left(\gamma_{0} n t\right)=f_{s}^{\circ}(p \bar{n})=$ $\delta_{P_{8}}(p) f_{s}^{\circ}(\bar{n})$. If $n$ is of the form

$$
n=\left(\begin{array}{ccc}
1 x(1+\theta) & w(1+\theta) & s^{\prime} \\
1 & s & y(1-\theta) \\
& -x(1+\theta) & 1
\end{array}\right)
$$


with $s^{\prime}=s+x y\left(1-\theta^{2}\right)$, then $\bar{n}=\chi\left(-\frac{x \theta^{2} \mathbb{N} b}{a_{1} b_{1}},-\frac{\lambda \theta^{2} y}{b_{1}^{2}}, \frac{\lambda \theta^{2} s^{\prime \prime}}{a_{1} b_{1}^{2}},-\frac{a_{2} \theta^{2}}{a_{1}},-\frac{b_{2} \theta^{2}}{b_{1}}\right)$ and $\psi(n)=$ $\psi_{\circ}\left(\theta^{2} x+y\right)$, where $s^{\prime \prime}=-b_{1} s+\left(b_{1}+b_{2}\right) x y \theta^{2}$. Since $\delta_{P_{8}}(p)=\left(\frac{\mathbb{N} a^{2} \mathbb{N} b^{2}}{a_{1}^{2} b_{1}^{4} \lambda}\right)^{3}$, the integral becomes

$$
\begin{aligned}
I\left(t, f_{s}^{\circ}, \psi\right)=\int_{F^{3}} \delta_{P_{8}}(p)^{\frac{s+3}{6}} f_{s}^{\circ}\left(\chi\left(-\frac{x \theta^{2} \mathbb{N} b}{a_{1} b_{1}},-\frac{\lambda \theta^{2} y}{b_{1}^{2}}, \frac{\lambda \theta^{2} s^{\prime \prime}}{a_{1} b_{1}^{2}},-\frac{a_{2} \theta^{2}}{a_{1}},-\frac{b_{2} \theta^{2}}{b_{1}}\right)\right) \\
\cdot \psi_{\circ}\left(\theta^{2} x+y\right) d x d y d s \\
=\left|\frac{\mathbb{N a} a^{2} \mathbb{N} b^{2}}{a_{1}^{2} b_{1}^{4} \lambda}\right|^{\frac{s+3}{2}} \int_{F^{3}} f_{s}^{\circ}\left(\chi\left(x, y, w,-\frac{a_{2} \theta^{2}}{a_{1}},-\frac{b_{2} \theta^{2}}{b_{1}}\right)\right) \\
\cdot \psi_{\circ}\left(-\frac{a_{1} b_{1}}{\mathbb{N} b} x-\frac{b_{1}^{2}}{\lambda \theta^{2}} y\right)\left|\frac{a_{1} b_{1}}{\theta^{2} \mathbb{N} b}\right| d x\left|\frac{b_{1}^{2}}{\lambda \theta^{2}}\right| d y\left|\frac{a_{1} b_{1}}{\lambda \theta^{2}}\right| d w
\end{aligned}
$$

after the change of variables $x \rightarrow-\frac{a_{1} b_{1}}{\theta^{2} \mathbb{N} b} x, y \rightarrow-\frac{b_{1}^{2}}{\lambda \theta^{2}} y$ and $s=-\frac{a_{1} b_{1}}{\lambda \theta^{2}}\left(w-\frac{b_{1}\left(b_{1}+b_{2}\right)}{\mathbb{N} b} x y\right)$. Simplifying now gives the result.

More explicitly, the images of $n$ and $t$ in $\operatorname{GSp}_{8}(F)$ are

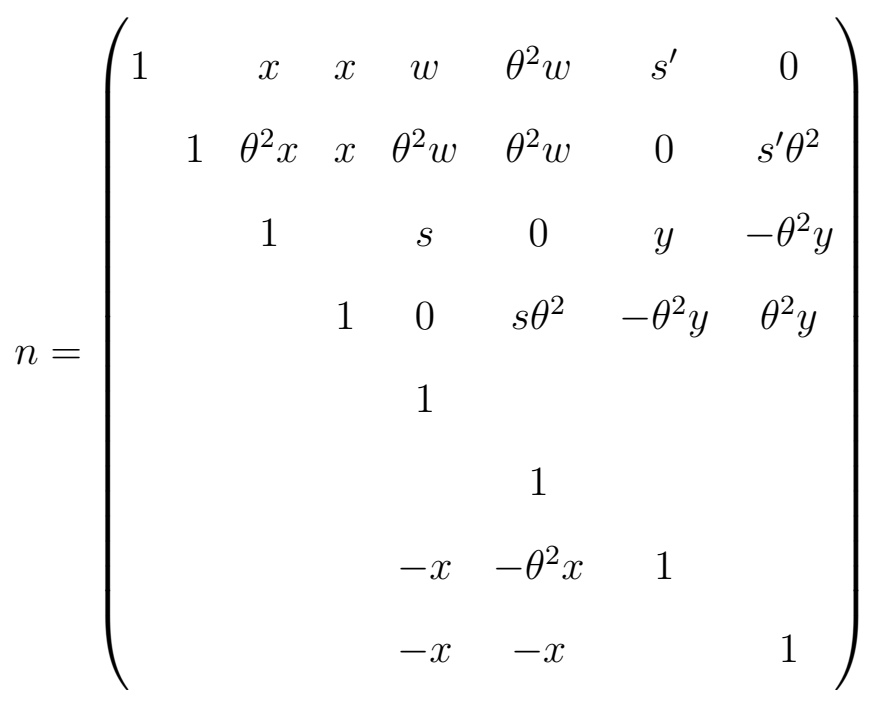


and

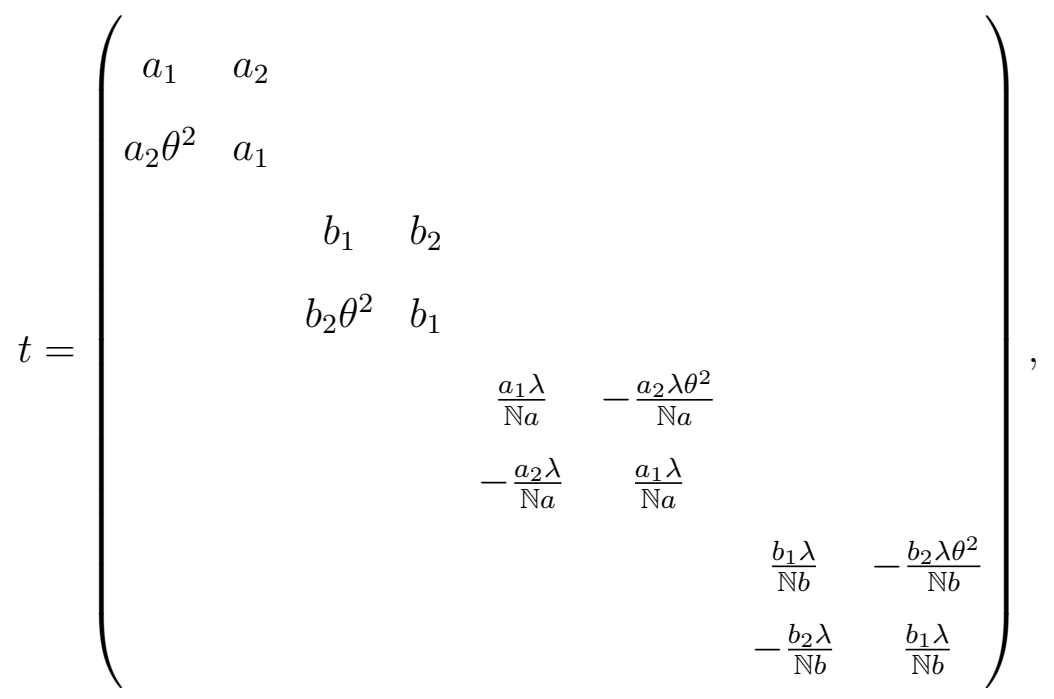

and we will have

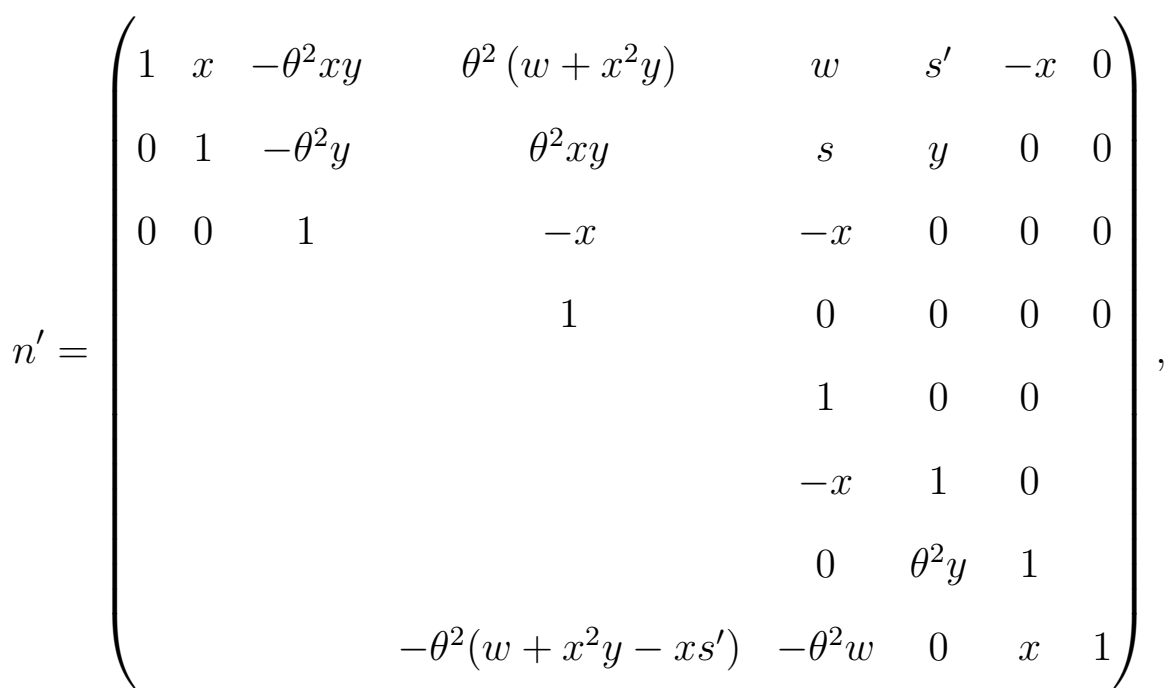

$$
\begin{aligned}
& p=\left(\begin{array}{ll}
A(p) & B(p) \\
C(p) & D(p)
\end{array}\right),
\end{aligned}
$$




$$
\begin{aligned}
& A(p)=\left(\begin{array}{cccc}
\frac{\mathbb{N} a}{a_{1}} & -\frac{a_{2} \theta^{2} x \mathbb{N} b}{a_{1} b_{1}} & \frac{a_{2} \lambda \theta^{2} s^{\prime \prime}}{a_{1} b_{1}^{2}} & 0 \\
0 & \frac{\mathbb{N} b}{b_{1}} & -\frac{b_{2} \lambda \theta^{2} y}{b_{1}^{2}} & \frac{b_{2} \lambda \theta^{2} s^{\prime \prime}}{a_{1} b_{1}^{2}} \\
0 & 0 & \frac{\lambda}{b_{1}} & -\frac{b_{2} \lambda \theta^{2} x}{a_{1} b_{1}} \\
0 & 0 & 0 & \frac{\lambda}{a_{1}}
\end{array}\right), \\
& B(p)=\left(\begin{array}{cccc}
0 & & & -a_{2} \\
& 0 & -b_{2} & \\
-\frac{b_{2} \lambda}{\mathbb{N} b} & 0 & \\
-\frac{a_{2} \lambda}{\mathbb{N} a} & & & 0
\end{array}\right), \\
& C(p)=\left(\begin{array}{cccc}
0 & & & \\
& & & \\
0 & & \\
& 0 & \\
& & & -\frac{b_{2} \lambda \theta^{4} x\left(b_{1}\left(s^{\prime}-x y\right)-s^{\prime \prime}\right)}{a_{1} b_{1}^{2}}
\end{array}\right), \\
& D(p)=\left(\begin{array}{cccc}
\frac{a_{1} \lambda}{\mathbb{N} a} & 0 & 0 & 0 \\
\frac{a_{2} \lambda \theta^{2} x}{\mathbb{N} a} & \frac{b_{1} \lambda}{\mathbb{N} b} & 0 & 0 \\
\frac{a_{2} \lambda \theta^{2}\left(s^{\prime}-x y\right)}{\mathbb{N} a} & \frac{b_{2} \lambda \theta^{2} y}{\mathbb{N} b} & b_{1} & 0 \\
0 & \frac{b_{2} \lambda \theta^{2}\left(s^{\prime}-x y\right)}{\mathbb{N} b} & b_{2} \theta^{2} x & a_{1}
\end{array}\right),
\end{aligned}
$$


and

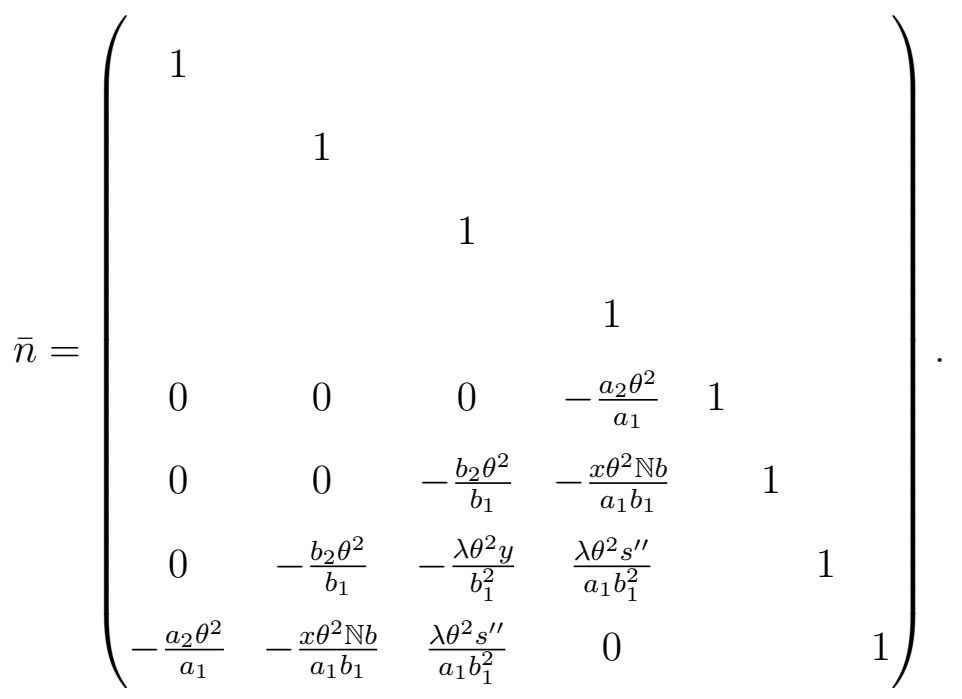

So it suffices to calculate the integral

$$
I\left(u, v, \alpha, \beta ; f_{s}^{\circ}, \psi_{\circ}\right)=\int_{F^{3}} f_{s}^{\circ}(\chi(x, y, w, u, v)) \psi_{\circ}(\alpha x+\beta y) d x d y d w .
$$

This is exactly the integral found in [24], II.3.2, equation (41). We give details of this calculation below.

\section{Lemma 3.1.3.}

$$
I\left(u, v, \alpha, \beta ; f_{s}^{\circ}, \psi_{\circ}\right)= \begin{cases}I\left(0,0, \alpha, \beta ; f_{s}^{\circ}, \psi_{\circ}\right) & \text { if }|u| \leq 1,|v| \leq 1, \\ |v|^{-2 s-2} I\left(0,0, v \alpha, v^{2} \beta ; f_{s}^{\circ}, \psi_{\circ}\right) & \text { if }|u| \leq 1,|v|>1, \\ |u|^{-s-1} I\left(0,0, u \alpha, \beta ; f_{s}^{\circ}, \psi_{\circ}\right) & \text { if }|u|>1,|v| \leq 1, \\ |u|^{-s-1}|v|^{-2 s-2} I\left(0,0, u v \alpha, v^{2} \beta ; f_{s}^{\circ}, \psi_{\circ}\right) & \text { if }|u|>1,|v|>1 .\end{cases}
$$


Proof. (i). If $|u|,|v| \leq 1$, then $\chi(x, y, w, u, v)=\chi(x, y, w) \chi_{-\epsilon_{1}-\epsilon_{4}}(u) \chi_{-\epsilon_{2}-\epsilon_{3}}(v)$, and the last two terms on the right are in $K^{\prime}=\operatorname{GSp}_{8}(\mathcal{O})$.

(ii). If $|u| \leq 1$ and $|v|>1$, then Lemma 3.1 .1 gives

$$
\begin{aligned}
& \chi(x, y, w, u, v)=\chi(x, y, w) \chi_{-\epsilon_{2}-\epsilon_{3}}(v) k_{u} \\
& \quad=\chi(x, y, w) h\left(1, v^{-1}, v^{-1}, 1,1, v, v, 1\right) \chi_{\epsilon_{2}+\epsilon_{3}}(v) k_{v} k_{u} \\
& \quad=h\left(1, v^{-1}, v^{-1}, 1,1, v, v, 1\right) \chi\left(v^{-1} x, v^{-2} y, v^{-1} w\right) \chi_{\epsilon_{2}+\epsilon_{3}}(v) k_{v} k_{u} \\
& \quad=h\left(1, v^{-1}, v^{-1}, 1,1, v, v, 1\right) p_{1}(x, y, w, v) \chi\left(v^{-1} x, v^{-2} y, v^{-1} w-v^{-2} x y\right) k_{u} k_{v} .
\end{aligned}
$$

for some $k_{u}, k_{v} \in K^{\prime}$. So the integral becomes

$$
\begin{aligned}
& I\left(u, v, \alpha, \beta ; f_{s}^{\circ}, \psi_{\circ}\right)=\int_{F^{3}} f_{s}^{\circ}(\chi(x, y, w, u, v)) \psi_{\circ}(\alpha x+\beta y) d x d y d w \\
& \quad=\int_{F^{3}}\left|\left(v^{-2}\right)^{6}\right|^{\frac{s}{6}+\frac{1}{2}} f_{s}^{\circ}\left(\chi\left(v^{-1} x, v^{-2} y, v^{-1} w-v^{-2} x y\right)\right) \psi_{\circ}(\alpha x+\beta y) d x d y d w \\
& =|v|^{-2 s-6} \int_{F^{3}} f_{s}^{\circ}(\chi(x, y, w)) \psi_{\circ}\left(\alpha v x+\beta v^{2} y\right)|v| d x\left|v^{2}\right| d y|v| d w \\
& =|v|^{-2 s-2} I\left(0,0, v \alpha, v^{2} \beta ; f_{s}^{\circ}, \psi_{\circ}\right) .
\end{aligned}
$$

(iii). If $|u|>1$ and $|v| \leq 1$, we have

$$
\begin{aligned}
& \chi(x, y, w, u, v)=\chi(x, y, w) \chi_{-\epsilon_{1}-\epsilon_{4}}(u) k_{v} \\
& \quad=\chi(x, y, w) h\left(u^{-1}, 1,1, u^{-1}, u, 1,1, u\right) \chi_{\epsilon_{1}+\epsilon_{4}}(u) k_{u} k_{v} \\
& \quad=h\left(u^{-1}, 1,1, u^{-1}, u, 1,1, u\right) \chi\left(u^{-1} x, y, u^{-1} w\right) \chi_{\epsilon_{1}+\epsilon_{4}}(u) k_{u} k_{v} \\
& \quad=h\left(u^{-1}, 1,1, u^{-1}, u, 1,1, u\right) p_{2}(x, y, w, u) \chi\left(u^{-1} x, y, u^{-1} w\right) k_{u} k_{v},
\end{aligned}
$$


for $k_{u}, k_{v} \in K^{\prime}$, giving the integral

$$
\begin{aligned}
& I\left(u, v, \alpha, \beta ; f_{s}^{\circ}, \psi_{\circ}\right) \\
& \quad=\int_{F^{3}}\left|\left(u^{-1}\right)^{6}\right|^{\frac{s}{6}+\frac{1}{2}} f_{s}^{\circ}\left(\chi\left(u^{-1} x, y, u^{-1} w\right)\right) \psi_{\circ}(\alpha x+\beta y) d x d y d w \\
& \quad=|u|^{-s-3} \int_{F^{3}} f_{s}^{\circ}(\chi(x, y, w)) \psi_{\circ}(\alpha u x+\beta y)|u| d x d y|u| d w \\
& \quad=|u|^{-s-1} I\left(0,0, u \alpha, \beta ; f_{s}^{\circ}, \psi_{\circ}\right) .
\end{aligned}
$$

(iv). If $|u|,|v|>1$, we will have

$$
\begin{aligned}
\chi(x, y, w, u, v)=\chi(x, y, w, u) \chi_{-\epsilon_{2}-\epsilon_{3}}(v) \\
=\chi(x, y, w, u) h\left(1, v^{-1}, v^{-1}, 1,1, v, v, 1\right) \chi_{\epsilon_{2}+\epsilon_{3}}(v) k_{v} \\
=h\left(1, v^{-1}, v^{-1}, 1,1, v, v, 1\right) \chi\left(v^{-1} x, v^{-2} y, v^{-1} w, u\right) \chi_{\epsilon_{2}+\epsilon_{3}}(v) k_{v} \\
=h\left(1, v^{-1}, v^{-1}, 1,1, v, v, 1\right) p_{3}(x, y, w, v) \\
\quad \chi\left(v^{-1} x, v^{-2} y, v^{-1} w-v^{-2} x y, u\right) k_{v},
\end{aligned}
$$

and

$$
\begin{aligned}
\chi & \left(v^{-1} x, v^{-2} y, v^{-1} s-v^{-2} x y, u\right)=\chi\left(v^{-1} x, v^{-2} y, v^{-1} s-v^{-2} x y\right) \chi_{-\epsilon_{1}-\epsilon_{4}}(u) \\
=\chi & \left(v^{-1} x, v^{-2} y, v^{-1} s-v^{-2} x y\right) h\left(u^{-1}, 1,1, u^{-1}, u, 1,1, u\right) \chi_{\epsilon_{1}+\epsilon_{4}}(u) k_{u} \\
= & h\left(u^{-1}, 1,1, u^{-1}, u, 1,1, u\right) p_{4}(x, y, w, u) \\
& \cdot \chi\left((u v)^{-1} x, v^{-2} y,(u v)^{-1}\left(w-v^{-1} x y\right)\right) k_{u},
\end{aligned}
$$


for $k_{u}, k_{v} \in K^{\prime}$, so the integral is

$$
\begin{aligned}
& I\left(u, v, \alpha, \beta ; f_{s}^{\circ}, \psi_{\circ}\right)=\left|u v^{2}\right|^{-s-3} \\
& \quad \cdot \int_{F^{3}} f_{s}^{\circ}\left(\chi\left((u v)^{-1} x, v^{-2} y,(u v)^{-1}\left(w-v^{-1} x y\right)\right)\right) \psi_{\circ}(\alpha x+\beta y) d x d y d w \\
& \quad=\left|u v^{2}\right|^{-s-3} \int_{F^{3}} f_{s}^{\circ}(\chi(x, y, w)) \psi_{\circ}\left(\alpha u v x+\beta v^{2} y\right)|u v| d x\left|v^{2}\right| d y|u v| d w \\
& \quad=|u|^{-s-1}|v|^{-2 s-2} I\left(0,0, u v \alpha, v^{2} \beta ; f_{s}^{\circ}, \psi_{\circ}\right) .
\end{aligned}
$$

Finally we compute the integral $I(\alpha, \beta)=I\left(0,0, \alpha, \beta ; f_{s}^{\circ}, \psi_{\circ}\right)$.

\section{Lemma 3.1.4.}

$$
\begin{aligned}
I(\alpha, \beta)= & {\left[\int_{|x| \leq 1} \psi_{\circ}(\alpha x) d x+\int_{|x|>1}|x|^{-s-2} \psi_{\circ}(\alpha x) d x\right] } \\
& \cdot\left[\int_{|y| \leq 1} \psi_{\circ}(\beta y) d y+\int_{|y|>1}|y|^{-s-2} \psi_{\circ}(\beta y) d y\right]\left[1+\int_{|w|>1}|w|^{-s-3} d w\right] .
\end{aligned}
$$

Proof. $I(\alpha, \beta)$ is absolutely convergent for $\operatorname{Re}(s)$ large, so fix $s$ in the domain of absolute convergence. Then we may freely change the order of integration since $F^{3}$ is abelian. So first change the order of integration to $d y d w d x$ and consider the integration in $y$. The integral over $\{|y| \leq 1\}$ is just

$$
\int_{|y| \leq 1} f_{s}^{\circ}(\chi(x, 0, w)) \psi_{\circ}(\beta y) d y=f_{s}^{\circ}(\chi(x, 0, w)) \int_{|y| \leq 1} \psi_{\circ}(\beta y) d y
$$

while the integral over $\{|y|>1\}$ becomes

$$
\begin{gathered}
\int_{|y|>1} f_{s}^{\circ}\left(\chi(x, 0, w) h\left(1, y^{-1}, 1,1,1, y, 1,1\right) \chi_{2 \epsilon_{3}}(y) k_{y}\right) \psi_{\circ}(\beta y) d y \\
\quad=\int_{|y|>1}|y|^{-s-3} f_{s}^{\circ}\left(\chi\left(x, 0, y^{-1} w\right) \chi_{2 \epsilon_{3}}(y)\right) \psi_{\circ}(\beta y) d y \\
\quad=\int_{|y|>1}|y|^{-s-3} f_{s}^{\circ}\left(\chi\left(x, 0, y^{-1} w\right)\right) \psi_{\circ}(\beta y) d y,
\end{gathered}
$$


where we used Lemma 3.1.1(viii),(i),(ii),(ix). Now exchange the $y$ - and $w$-integration and change variables $w \rightarrow y w$. This introduces a factor of $|y|$, so $I(\alpha, \beta)$ becomes

$$
\begin{gathered}
I(\alpha, \beta)=\int_{F} \psi_{\circ}(\alpha x)\left[\int_{|y| \leq 1} \psi_{\circ}(\beta y)\left(\int_{F} f_{s}^{\circ}(\chi(x, 0, w)) d w\right) d y\right. \\
\left.\quad+\int_{|y|>1}|y|^{-s-3} \psi_{\circ}(\beta y)\left(\int_{F} f_{s}^{\circ}\left(\chi\left(x, 0, y^{-1}(y w)\right)\right)|y| d w\right) d y\right] d x \\
=\left[\int_{|y| \leq 1} \psi_{\circ}(\beta y) d y+\int_{|y|>1}|y|^{-s-2} \psi_{\circ}(\beta y) d y\right]\left[\int_{F^{2}} f_{s}^{\circ}(\chi(x, 0, w)) \psi_{\circ}(\alpha x) d x d w\right] .
\end{gathered}
$$

The other two integrations are worked out similarly.

Lemma 3.1.5. Let $\tilde{\psi}$ be an additive character of $F$ with conductor $\mathcal{O}, \alpha \in F$ with $\operatorname{ord}_{v}(\alpha)=a$ and $s \in \mathbb{C}$. Then for $\operatorname{Re}(s)>>0$,

(i). $\int_{|x|>1}|x|^{-s-2} \tilde{\psi}(\alpha x) d x=-1+\left(1-q^{-s-2}\right) \frac{1-q^{-(a+1)(s+1)}}{1-q^{-s-1}}$, if $a \geq 0$, and is 0 otherwise;

(ii). $1+\int_{|w|>1}|w|^{-s-3} d w=\frac{1-q^{-s-3}}{1-q^{-s-2}}$.

Proof. Standard calculations.

Using Lemma 3.1.5, it is easy to see that if $A=\operatorname{ord}_{v} \alpha, B=\operatorname{ord}_{v} \beta$, then

$$
I(\alpha, \beta)=\frac{\left(1-q^{-s-3}\right)\left(1-q^{-s-2}\right)}{\left(1-q^{-s-1}\right)^{2}}\left(1-q^{-(s+1)(A+1)}\right)\left(1-q^{-(s+1)(B+1)}\right)
$$

for $|\alpha|,|\beta| \leq 1$ and $I(\alpha, \beta)$ is zero otherwise.

We also note for later reference that we can calculate $I\left(0,0, \alpha, \beta ; f_{s}^{\circ}, 1\right)$ for $\psi_{\circ}=1$ : the integrals $\int_{\mathcal{O}} \psi_{\circ}(\alpha x) d x$ and $\int_{\mathcal{O}} \psi_{\circ}(\beta y) d y$ are both 1 , and so the $x$-integrals become

$$
1+\int_{|x|>1}|x|^{-s-2} d x=\frac{1-q^{-s-2}}{1-q^{-s-1}}
$$


by Lemma 3.1.5(ii), and the $y$-integrals are identical. The result, after cancellation, is

$$
I\left(0,0, \alpha, \beta ; f_{s}^{\circ}, 1\right)=\frac{\left(1-q^{-s-2}\right)\left(1-q^{-s-3}\right)}{\left(1-q^{-s-1}\right)^{2}}=\frac{\zeta_{v}(s+1)^{2}}{\zeta_{v}(s+2) \zeta_{v}(s+3)} .
$$

Now we will fix a parametrization of $Z(F) \backslash T_{0}(F)$ by taking as a set of representatives elements

$$
t=t^{\prime}\left(a_{1}, a_{2}, b_{1}, b_{2}\right) \stackrel{\text { def }}{=} h\left(a_{1}\left(1+a_{2} \theta\right), b_{1}\left(1+b_{2} \theta\right), \lambda_{a, b}\left(1-a_{2} \theta\right), 1-b_{2} \theta\right),
$$

where

$$
\lambda_{a, b}=\frac{b_{1}\left(1-b_{2}^{2} \theta^{2}\right)}{a_{1}\left(1-a_{2}^{2} \theta^{2}\right)}
$$

(Note that this actually parametrizes the open subset of elements $h(a, b, c, d)$ in $T_{0}(F)$ with $d=d_{1}+d_{2} \theta$ and $d_{1} \neq 0$, but its complement has measure zero, so we may use this parametrization in our integral.)

For convenience, define a function $\delta: \mathbb{Z} \rightarrow \mathbb{Z}$ by

$$
\delta(m)= \begin{cases}0 & \text { if } m>0 \\ m & \text { if } m \leq 0\end{cases}
$$

Then we have the following

Lemma 3.1.6. For $t=t^{\prime}\left(a_{1}, a_{2}, b_{1}, b_{2}\right)$ as above and $m_{i}=\operatorname{ord}_{v} a_{i}, n_{i}=\operatorname{ord}_{v} b_{i}$, $\delta_{B_{4}}(t)=q^{-3 n_{1}-4\left(m_{1}+\delta\left(m_{2}\right)-n_{1}-\delta\left(n_{2}\right)\right)}$.

Assembling the above results gives us the inner integral: 
Proposition 3.1.7. For $t=t^{\prime}\left(a_{1}, a_{2}, b_{1}, b_{2}\right)$ as above, let $m_{i}=\operatorname{ord}_{v} a_{i}, n_{i}=\operatorname{ord}_{v} b_{i}$ for $i=1,2$. Then

$$
\begin{gathered}
I\left(t, f_{s}^{\circ}, \psi\right)=\zeta_{v}(s+1)^{2} \zeta_{v}(s+2)^{-1} \zeta_{v}(s+3)^{-1} q^{-(s+5)\left(m_{1}+\delta\left(m_{2}\right)-\delta\left(n_{2}\right)\right)+\left(\frac{s+3}{2}\right) n_{1}} \\
\cdot\left(1-q^{-(s+1)\left(1+n_{1}\right)}\right)\left(1-q^{-(s+1)\left(1+m_{1}+\delta\left(m_{2}\right)-n_{1}-\delta\left(n_{2}\right)\right)}\right), \\
\quad \text { if } m_{1}+\delta\left(m_{2}\right)-\delta\left(n_{2}\right) \geq n_{1} \geq 0
\end{gathered}
$$

and is equal to 0 otherwise. More explicitly,

$$
\begin{aligned}
& I\left(t, f_{s}^{\circ}, \psi\right)=\zeta_{v}(s+1)^{2} \zeta_{v}(s+2)^{-1} \zeta_{v}(s+3)^{-1} q^{-(s+5) m_{1}+\left(\frac{s+3}{2}\right) n_{1}}\left(1-q^{-(s+1)\left(1+n_{1}\right)}\right) \\
& \cdot\left\{\begin{array}{l}
\left(1-q^{-(s+1)\left(1+m_{1}-n_{1}\right)}\right) \quad \text { if } m_{2} \geq 0, n_{2} \geq 0, m_{1} \geq n_{1} \geq 0, \\
q^{(s+5) n_{2}}\left(1-q^{-(s+1)\left(1+m_{1}-n_{1}-n_{2}\right)}\right) \\
\text { if } m_{2} \geq 0, n_{2}<0, m_{1}-n_{2} \geq n_{1} \geq 0, \\
q^{-(s+5) m_{2}}\left(1-q^{-(s+1)\left(1+m_{1}+m_{2}-n_{1}\right)}\right) \\
\text { if } m_{2}<0, n_{2} \geq 0, m_{1}+m_{2} \geq n_{1} \geq 0, \\
q^{-(s+5)\left(m_{2}-n_{2}\right)}\left(1-q^{-(s+1)\left(1+m_{1}+m_{2}-n_{1}-n_{2}\right)}\right) \\
\text { if } m_{2}<0, n_{2}<0, m_{1}+m_{2}-n_{2} \geq n_{1} \geq 0 \\
\text { otherwise. }
\end{array}\right.
\end{aligned}
$$

Proof. Change variables $a_{2} \rightarrow a_{1} a_{2}, b_{2} \rightarrow b_{1} b_{2}$ in (3.4) and set $a=1+a_{2} \theta, b=1+b_{2} \theta$, so that $\mathbb{N} a \rightarrow a_{1}^{2} \mathbb{N} a, \mathbb{N} b \rightarrow b_{1}^{2} \mathbb{N} b, \lambda \rightarrow b_{1} \mathbb{N} b$; then by Lemma 3.1.2 and Lemma 3.1.3 
we have

$$
\begin{aligned}
& I\left(t, f_{s}^{\circ}, \psi_{\circ}\right)=\left|a_{1}\right|^{s+5}\left|b_{1}\right|^{-\frac{s+3}{2}}|\mathbb{N} a|^{s+3}|\mathbb{N} b|^{\frac{s-3}{2}} \\
& \cdot I\left(-a_{2} \theta^{2},-b_{2} \theta^{2},-\frac{a_{1}}{b_{1} \mathbb{N} b},-\frac{b_{1}}{\theta^{2} \mathbb{N} b} ; f_{s}^{\circ}, \psi_{\circ}\right) \\
& =C\left\{\begin{array}{cc}
I\left(-\frac{a_{1}}{b_{1} \mathbb{N} b},-\frac{b_{1}}{\theta^{2} \mathbb{N} b}\right) & \text { if } m_{2} \geq 0, n_{2} \geq 0, \\
\left|b_{2}\right|^{-2 s-2} I\left(\left(-b_{2} \theta^{2}\right)\left(-\frac{a_{1}}{b_{1} \mathbb{N} b}\right),\left(-b_{2} \theta^{2}\right)^{2}\left(-\frac{b_{1}}{\theta^{2} \mathbb{N} b}\right)\right) & \text { if } m_{2} \geq 0, n_{2}<0, \\
\left|a_{2}\right|^{-s-1} I\left(\left(-a_{2} \theta^{2}\right)\left(-\frac{a_{1}}{b_{1} \mathbb{N} b}\right),-\frac{b_{1}}{\theta^{2} \mathbb{N} b}\right) & \text { if } m_{2}<0, n_{2} \geq 0, \\
\left|a_{2}\right|^{-s-1}\left|b_{2}\right|^{-2 s-2} I\left(\left(a_{2} b_{2} \theta^{4}\right)\left(-\frac{a_{1}}{b_{1} \mathbb{N} b}\right),\left(-b_{2} \theta^{2}\right)^{2}\left(-\frac{b_{1}}{\theta^{2} \mathbb{N} b}\right)\right) & \text { if } m_{2}<0, n_{2}<0 .
\end{array}\right.
\end{aligned}
$$

where $C=C\left(a_{i}, b_{i} ; s\right)=\left|a_{1}\right|^{s+5}\left|b_{1}\right|^{-\frac{s+3}{2}}|\mathbb{N} a|^{s+3}|\mathbb{N} b|^{\frac{s-3}{2}}$. Observing that $|\mathbb{N} a|=1$ if $\left|a_{2}\right| \leq 1$ and $|\mathbb{N} a|=\left|a_{2}\right|^{2}$ if $\left|a_{2}\right|>1$ gives

$$
C=\left|a_{1}\right|^{s+5}\left|b_{1}\right|^{-\frac{s+3}{2}} \max \left\{1,\left|a_{2}\right|\right\}^{2 s+6} \max \left\{1,\left|b_{2}\right|\right\}^{s-3}
$$

and so the coefficient of $I(\alpha, \beta)$ in each case is

$$
\begin{gathered}
C \cdot \max \left\{1,\left|a_{2}\right|\right\}^{-s-1} \max \left\{1,\left|b_{2}\right|\right\}^{-2 s-2} \\
=\left|a_{1}\right|^{s+5}\left|b_{1}\right|^{-\frac{s+3}{2}} \max \left\{1,\left|a_{2}\right|\right\}^{s+5} \max \left\{1,\left|b_{2}\right|\right\}^{-s-5} \\
=q^{-(s+5) m_{1}+\left(\frac{s+3}{2}\right) n_{1}-\delta\left(m_{2}\right)(s+5)+\delta\left(n_{2}\right)(s+5)}
\end{gathered}
$$

Now applying (3.5) gives the result.

Remark 3.1.8. Note that Lemma 3.1.2 and Lemma 3.1.3 make no assumption on $\psi$ 
or $\psi_{\circ}$ : it is only in Lemma 3.1.4 that $\psi_{\text {。 }}$ is assumed to have conductor $\mathcal{O}$. If we take $\psi_{\circ}$ and hence $\psi$ to be trivial and use (3.6), following the proof above gives

$$
\begin{aligned}
I\left(t, f_{s}^{\circ}, 1\right) & =|\theta|^{-6} C\left(a_{i}, b_{i} ; s\right) \max \left\{1,\left|a_{2}\right|\right\}^{-s-1} \max \left\{1,\left|b_{2}\right|\right\}^{-2 s-2} \frac{\zeta_{v}(s+1)^{2}}{\zeta_{v}(s+2) \zeta_{v}(s+3)} \\
& =|\theta|^{-6}\left|a_{1}\right|^{s+5}\left|b_{1}\right|^{-\frac{s+3}{2}} \max \left\{1,\left|a_{2}\right|\right\}^{s+5} \max \left\{1,\left|b_{2}\right|\right\}^{-s-5} \frac{\zeta_{v}(s+1)^{2}}{\zeta_{v}(s+2) \zeta_{v}(s+3)} \\
& =|\theta|^{-6}\left|a_{1}\right|^{s+5}\left|b_{1}\right|^{-\frac{s+3}{2}}|\mathbb{N} a|^{\frac{s+5}{2}}|\mathbb{N} b|^{-\frac{s+5}{2}} \frac{\zeta_{v}(s+1)^{2}}{\zeta_{v}(s+2) \zeta_{v}(s+3)}
\end{aligned}
$$

where we have inserted $|\theta|$ for clarity.

\subsubsection{The Unramified Integral}

We need the following formula for the unramified Whittaker function on $\mathrm{GSp}_{4 / E}$, found in Casselman-Shalika [5]. Since we are dealing with a split group, we may use the simpler formula found in Bump's survey [3]; see also [24], Thm. I.3.3.1.

Theorem 3.1.9 ([5]). Let $t=t^{\prime}\left(a_{1}, a_{2}, b_{1}, b_{2}\right)$ as above, with $m_{i}=\operatorname{ord}_{v} a_{i}, n_{i}=\operatorname{ord}_{v} b_{i}$ for $i=1,2$. The spherical Whittaker function $W^{\circ}=W_{E}^{\circ}$ for $\pi$ is given by

$$
W^{\circ}(t)= \begin{cases}\delta_{E}^{\frac{1}{2}}(t) \operatorname{tr} \tau_{\left(n_{1}, m_{1}+\delta\left(m_{2}\right)-n_{1}-\delta\left(n_{2}\right)\right)}\left(z_{\pi}\right) \\ & \text { if } m_{1}+\delta\left(m_{2}\right)-\delta\left(n_{2}\right) \geq n_{1} \geq 0 \\ 0 & \text { otherwise }\end{cases}
$$

where $\delta_{E}$ is the modulus character of the Borel subgroup $B_{4}(E)$ in $\operatorname{GSp}_{4}(E)$ and $\operatorname{tr} \tau_{(m, n)}\left(z_{\pi}\right)$ is the character of the representation $\tau_{(m, n)}$ of $\operatorname{Sp}_{4}(\mathbb{C})$ evaluated at the Satake parameter $z_{\pi}$ of $\pi$, with $\tau_{(m, n)}$ as in Prop. 2.2.3. 
Finally, we can evaluate the unramified integral.

Theorem 3.1.10. The normalized local integral is

$$
\begin{aligned}
\Psi^{*}\left(s, W^{\circ}, f_{s}^{\circ}\right)=d_{G, v}(s) \Psi\left(s, W^{\circ}, f_{s}^{\circ}\right) \\
\quad=\frac{1}{\left(1-X^{2}\right)^{3}\left(1-X^{4}\right)} \sum_{k, l \geq 0} \operatorname{tr} \tau_{(k, l)}\left(z_{\pi}\right) X^{k+2 l}\left(1-\left(X^{2}\right)^{k+1}\right)\left(1-\left(X^{2}\right)^{l+1}\right),
\end{aligned}
$$

where $X=q^{-\frac{s+1}{2}}$ and $d_{G, v}(s)=\zeta_{v}(2 s+2) \zeta_{v}(s+1) \zeta_{v}(s+2) \zeta_{v}(s+3)$ is the local component of the normalizing factor for the Eisenstein series $E\left(g, s, f_{s}\right)$.

Proof. We break up the integral over $Z(F) \backslash T_{0}(F)$ into four pieces, corresponding to the cases in Prop. 3.1.7. For $\eta_{1}, \eta_{2}= \pm$, set

$$
T_{\eta_{1}, \eta_{2}}=\left\{t=t^{\prime}\left(a_{1}, a_{2}, b_{1}, b_{2}\right) \mid \operatorname{sgn} \operatorname{ord}_{v} a_{2}=\eta_{1}, \operatorname{sgn} \operatorname{ord}_{v} b_{2}=\eta_{2}\right\},
$$

with the convention that $\operatorname{sgn} 0=+$. Then $T_{\eta_{1}, \eta_{2}} \simeq \mathbb{Z}^{2} \times K_{\eta_{1}, \eta_{2}}$, where $K_{\eta_{1}, \eta_{2}} \subseteq T_{0}(\mathcal{O})$ and the $\mathbb{Z}^{2}$-part is given by the $v$-adic orders of the simple roots. In fact, by setting $\bar{c}=\varpi^{\operatorname{ord}_{v} c}$ for $c \in F$, we see that $t \in T_{\eta_{1}, \eta_{2}}$ has one of the following forms (up to an element of $\left.K_{\eta_{1}, \eta_{2}}\right)$ :

$$
\begin{array}{lll}
\left(\eta_{1}, \eta_{2}\right)=(+,+) & \Rightarrow & t=h\left(\bar{a}_{1}, \bar{b}_{1}, \bar{a}_{1}^{-1} \bar{b}_{1}, 1\right) ; \\
\left(\eta_{1}, \eta_{2}\right)=(+,-) & \Rightarrow & t=h\left(\bar{a}_{1}, \bar{b}_{1} \bar{b}_{2}, \bar{a}_{1}^{-1} \bar{b}_{1} \bar{b}_{2}^{2}, \bar{b}_{2}\right) ; \\
\left(\eta_{1}, \eta_{2}\right)=(-,+) & \Rightarrow & t=h\left(\bar{a}_{1} \bar{a}_{2}, \bar{b}_{1},\left(\bar{a}_{1} \bar{a}_{2}\right)^{-1} \bar{b}_{1}, 1\right) ; \\
\left(\eta_{1}, \eta_{2}\right)=(-,-) & \Rightarrow & t=h\left(\bar{a}_{1} \bar{a}_{2}, \bar{b}_{1} \bar{b}_{2},\left(\bar{a}_{1} \bar{a}_{2}\right)^{-1} \bar{b}_{1} \bar{b}_{2}^{2}, \bar{b}_{2}\right) .
\end{array}
$$


By considering the variants of $t^{\prime}\left(a_{1}, a_{2}, b_{1}, b_{2}\right)$ given by

$$
\begin{gathered}
t_{1}^{\prime}\left(a_{1}, a_{2}, b_{1}, b_{2}\right)=h\left(a_{1}\left(a_{2}+\theta\right), b_{1}\left(1+b_{2} \theta\right), \lambda_{a, b}^{(1)}\left(a_{2}-\theta\right), 1-b_{2} \theta\right), \\
t_{2}^{\prime}\left(a_{1}, a_{2}, b_{1}, b_{2}\right)=h\left(a_{1}\left(1+a_{2} \theta\right), b_{1}\left(b_{2}+\theta\right), \lambda_{a, b}^{(2)}\left(1-a_{2} \theta\right), b_{2}-\theta\right), \\
t_{1,2}^{\prime}\left(a_{1}, a_{2}, b_{1}, b_{2}\right)=h\left(a_{1}\left(a_{2}+\theta\right), b_{1}\left(b_{2}+\theta\right), \lambda_{a, b}^{(1,2)}\left(a_{2}-\theta\right), b_{2}-\theta\right),
\end{gathered}
$$

with similitudes defined appropriately, the $K_{\eta_{1}, \eta_{2}}$ may be described as follows:

$$
\begin{aligned}
& K_{++}=\left\{t^{\prime}\left(u_{1}, a_{2}, v_{1}, b_{2}\right) \mid a_{2}, b_{2} \in \mathcal{O}, u_{1}, v_{1} \in \mathcal{O}^{\times}\right\}, \\
& K_{+-}=\left\{t_{2}^{\prime}\left(u_{1}, a_{2}, v_{1}, b_{2}\right) \mid a_{2}, b_{2} \in \mathcal{O}, u_{1}, v_{1} \in \mathcal{O}^{\times}\right\}, \\
& K_{-+}=\left\{t_{1}^{\prime}\left(u_{1}, a_{2}, v_{1}, b_{2}\right) \mid a_{2}, b_{2} \in \mathcal{O}, u_{1}, v_{1} \in \mathcal{O}^{\times}\right\}, \\
& K_{--}=\left\{t_{1,2}^{\prime}\left(u_{1}, a_{2}, v_{1}, b_{2}\right) \mid a_{2}, b_{2} \in \mathcal{O}, u_{1}, v_{1} \in \mathcal{O}^{\times}\right\} .
\end{aligned}
$$

Normalize the measure on $Z(F) \backslash T_{0}(F)$ so that $\operatorname{vol}\left(Z(F) \backslash Z(F) T_{0}(\mathcal{O})\right)=1$. Then we easily compute that

$$
\begin{gathered}
\operatorname{vol}\left(Z(F) \backslash Z(F) K_{++}\right)=\frac{q^{2}}{(q+1)^{2}}, \quad \operatorname{vol}\left(Z(F) \backslash Z(F) K_{--}\right)=\frac{1}{(q+1)^{2}} \\
\operatorname{vol}\left(Z(F) \backslash Z(F) K_{+-}\right)=\operatorname{vol}\left(Z(F) \backslash Z(F) K_{-+}\right)=\frac{q}{(q+1)^{2}}
\end{gathered}
$$


Since $\delta_{B_{0}}^{-1}(t) \delta_{E}^{\frac{1}{2}}(t)=\delta_{B_{4}}^{-2}(t) \delta_{B_{4}}(t)=\delta_{B_{4}}^{-1}(t)$, using Prop. 3.1.7 and Theorem 3.1.9 gives

$$
\begin{aligned}
& \Psi\left(s, W^{\circ}, f_{s}^{\circ}\right)=\zeta_{v}(s+1)^{2} \zeta_{v}(s+2)^{-1} \zeta_{v}(s+3)^{-1} \\
& \cdot\left[\operatorname{vol}\left(K_{++}\right) \sum_{\substack{m_{1}-n_{1} \geq 0 \\
n_{1} \geq 0}} \operatorname{tr} \tau_{\left(n_{1}, m_{1}-n_{1}\right)}\left(z_{\pi}\right) P_{1}\left(m_{1}, m_{2}, n_{1}, n_{2}, X\right)\right. \\
& \quad+\operatorname{vol}\left(K_{-+}\right) \sum_{\substack{m_{1}+m_{2}-n_{1} \geq 0 \\
n_{1} \geq 0}} \operatorname{tr} \tau_{\left(n_{1}, m_{1}+m_{2}-n_{1}\right)}\left(z_{\pi}\right) P_{2}\left(m_{1}, m_{2}, n_{1}, n_{2}, X\right) \\
& \quad+\operatorname{vol}\left(K_{+-}\right) \sum_{\substack{m_{1}-n_{1}-n_{2} \geq 0 \\
n_{1} \geq 0}} \operatorname{tr} \tau_{\left(n_{1}, m_{1}-n_{1}-n_{2}\right)}\left(z_{\pi}\right) P_{3}\left(m_{1}, m_{2}, n_{1}, n_{2}, X\right) \\
& \left.\quad+\operatorname{vol}\left(K_{--}\right) \sum_{\substack{m_{1}+m_{2}-n_{1}-n_{2} \geq 0 \\
n_{1} \geq 0}} \operatorname{tr} \tau_{\left(n_{1}, m_{1}+m_{2}-n_{1}-n_{2}\right)}\left(z_{\pi}\right) P_{4}\left(m_{1}, m_{2}, n_{1}, n_{2}, X\right)\right],
\end{aligned}
$$

where $X=q^{-\frac{s+1}{2}}$ and

$$
\begin{gathered}
P_{1}\left(m_{1}, m_{2}, n_{1}, n_{2}, X\right)=X^{2 m_{1}-n_{1}}\left(1-X^{2\left(1+n_{1}\right)}\right) \\
\cdot\left(1-X^{2\left(1+m_{1}-n_{1}\right)}\right) \\
P_{2}\left(m_{1}, m_{2}, n_{1}, n_{2}, X\right)=X^{2 m_{1}+2 m_{2}-n_{1}}\left(1-X^{2\left(1+n_{1}\right)}\right) \\
\cdot\left(1-X^{2\left(1+m_{1}+m_{2}-n_{1}\right)}\right) \\
P_{3}\left(m_{1}, m_{2}, n_{1}, n_{2}, X\right)=X^{2 m_{1}-2 n_{2}-n_{1}}\left(1-X^{2\left(1+n_{1}\right)}\right) \\
\cdot\left(1-X^{2\left(1+m_{1}-n_{1}-n_{2}\right)}\right) \\
P_{4}\left(m_{1}, m_{2}, n_{1}, n_{2}, X\right)=X^{2 m_{1}+2 m_{2}-n_{1}-2 n_{2}}\left(1-X^{2\left(1+n_{1}\right)}\right) \\
\cdot\left(1-X^{2\left(1+m_{1}+m_{2}-n_{1}-n_{2}\right)}\right) .
\end{gathered}
$$

Now we substitute the volumes and change variables $n_{1} \rightarrow k, m_{1}+\delta\left(m_{2}\right)-n_{1}-\delta\left(n_{2}\right) \rightarrow$ 
$l$, noting that all four sums collapse to the single sum in (3.10). Finally, multiplying by the normalizing factor $d_{G, v}(s)$ gives us what we want.

\subsubsection{Local $L$-factor}

We need to identify our local integral with a local Langlands $L$-factor, in this case that associated with the twisted tensor representation defined as follows.

As in [2], the dual group of $\mathrm{GSp}_{4}$ is $\mathrm{GSp}_{4}(\mathbb{C})$, and if $\Gamma_{F}$ is the absolute Galois group of $F$, then

$$
\begin{aligned}
{ }^{L} G_{0} & =\left(\operatorname{GSp}_{4}(\mathbb{C}) \times \operatorname{GSp}_{4}(\mathbb{C})\right)^{\circ} \rtimes \Gamma_{F} \\
& =\left(\left(\operatorname{GSp}_{4}(\mathbb{C}) \times \operatorname{GSp}_{4}(\mathbb{C})\right)^{\circ} \rtimes \Gamma_{E / F}\right) \times \Gamma_{E},
\end{aligned}
$$

where $\Gamma_{E / F}=\Gamma_{F} / \Gamma_{E}$. The superscript ${ }^{\circ}$ here denotes the similitude of each factor is the same. This follows from the general discussion of $L$-groups of groups arising from restriction of scalars in $[2, \mathrm{I} .4,5]$. We also find that if $\mathrm{GL}_{4 / E}$ is the group $\mathrm{GL}_{4}$ considered as a group over $E$ and $H=\operatorname{Res}_{E / F} \mathrm{GL}_{4 / E}$, then

$$
{ }^{L} H=\left(\mathrm{GL}_{4}(\mathbb{C}) \times \mathrm{GL}_{4}(\mathbb{C})\right) \rtimes \Gamma_{E / F} .
$$

Let $(\rho, V)$ be the defining representation for $\mathrm{Sp}_{4}(\mathbb{C})$, and extend $\rho$ to $\mathrm{GSp}_{4}(\mathbb{C})$ trivially on similitudes. That is, write $g \in \mathrm{GSp}_{4}(\mathbb{C})$ as

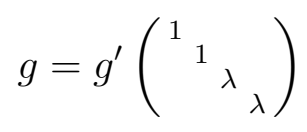

for $g^{\prime} \in \mathrm{Sp}_{4}(\mathbb{C})$ and define $\rho(g)=\rho\left(g^{\prime}\right)$. Then $\rho: \mathrm{GSp}_{4}(\mathbb{C}) \rightarrow \mathrm{GL}_{4}(\mathbb{C})$. Let $\sigma$ generate 
the group $\Gamma_{E / F}$. Then the twisted tensor representation $\rho_{\mathrm{TT}}^{\prime}:{ }^{L} H \rightarrow \mathrm{GL}(V \otimes V) \simeq$ $\mathrm{GL}_{16}(\mathbb{C})$ is given by

$$
\begin{aligned}
& \rho_{\mathrm{TT}}^{\prime}\left(g_{1}, g_{2}\right): x \otimes y \mapsto x g_{1} \otimes y g_{2}, \\
& \rho_{\text {TT }}^{\prime}(\sigma): x \otimes y \mapsto y \otimes x,
\end{aligned}
$$

for $x, y \in V$ and $g_{1}, g_{2} \in \mathrm{GL}_{4}(\mathbb{C})$. We define the twisted tensor representation of $\mathrm{GSp}_{4}$ by the composition

$$
\rho_{\mathrm{TT}}: \mathrm{GSp}_{4}(\mathbb{C}) \stackrel{\rho}{\rightarrow} \mathrm{GL}_{4}(\mathbb{C}) \stackrel{\rho_{\mathrm{TT}}^{\prime}}{\longrightarrow} \mathrm{GL}_{16}(\mathbb{C})
$$

so that

$$
\begin{aligned}
& \rho_{\mathrm{TT}}\left(g_{1}, g_{2}\right): x \otimes y \mapsto \rho\left(g_{1}\right) x \otimes \rho\left(g_{2}\right) y, \\
& \rho_{\mathrm{TT}}(\sigma): x \otimes y \mapsto y \otimes x,
\end{aligned}
$$

for $\left(g_{1}, g_{2}\right) \in\left(\operatorname{GSp}_{4}(\mathbb{C}) \times \operatorname{GSp}_{4}(\mathbb{C})\right)^{\circ}$.

Since the group $G=\operatorname{GSp}_{4}(E)$ is split over $E$, its $L$-group is ${ }^{L} G=\operatorname{GSp}_{4}(\mathbb{C}) \times \Gamma_{E}$. The Satake parameter for $\pi$ is a conjugacy class which can be represented by a semisimple element $z^{\prime}=\operatorname{diag}\left(z_{1}, \ldots, z_{4}\right)$. The $L$-homomorphism

$$
r_{E / F}:\left\{\begin{array}{l}
{ }^{L} G=\mathrm{GSp}_{4}(\mathbb{C}) \times \Gamma_{E} \rightarrow{ }^{L} G_{0}, \\
g \times \tau \mapsto(g, 1) \rtimes \iota(\tau),
\end{array}\right.
$$

where $\iota: \Gamma_{E} \hookrightarrow \Gamma_{F}$ is the natural inclusion, arises from restriction of scalars. Then the representative of $z^{\prime}$ in ${ }^{L} G_{0}$ is $z=r_{E / F}\left(z^{\prime}\right) \rtimes \sigma$. It is an easy exercise to check that

$$
L\left(s, \pi, \rho_{\mathrm{TT}}\right)=\operatorname{det}\left(I-\rho_{\mathrm{TT}}(z) q^{-s}\right)^{-1}=\prod_{i=1}^{4}\left(1-z_{i} q^{-s}\right)^{-1} \prod_{1 \leq i<j \leq 4}\left(1-z_{i} z_{j} q^{-2 s}\right)^{-1} .
$$


The method of identifying this local $L$-factor with the unramified integral is as follows. First, in [34] we find a formula that relates the right-hand side of (3.14) to classical Schur functions, which are the characters of finite-dimensional representations of $\mathrm{GL}_{4}(\mathbb{C})$. Our motivation for using this is $[8, \mathrm{p}$. 305]. Second, we use a branching formula of Koike-Terada [28] to relate characters of $\mathrm{GL}_{4}(\mathbb{C})$ to those of $\mathrm{Sp}_{4}(\mathbb{C})$. This latter formula involves sums of Littlewood-Richardson coefficents. Finally, we evaluate these coefficients and relate them to the rational functions found in Prop. 3.1.10, which will give the desired equality. We should point out that this approach through restriction of characters first came to our attention through a note in [13] pointing to work of Sundaram [42, 41], which we consulted first and from which we greatly benefitted. Ultimately [28] was more easily adaptable to our work here.

Let us begin by recalling some definitions from [34].

A partition $\lambda$ is a sequence $\lambda=\left(\lambda_{1}, \lambda_{2}, \ldots, \lambda_{k}, \ldots\right)$ of non-negative integers such that $\lambda_{1} \geq \lambda_{2} \geq \cdots \geq \lambda_{k} \geq \cdots$ and such that there is an $r$ satisfying $\lambda_{k}=0$ for all $k>r$. The smallest such $r$ is the length of $\lambda$, denoted $\ell(\lambda)$. The nonzero $\lambda_{i}$ are the parts of $\lambda$, and the sum of the parts of $\lambda$ is the size of $\lambda$, written $|\lambda|$. If $|\lambda|=n$, we say $\lambda$ is a partion of $n$ and write $\lambda \vdash n$. A partition is called even if all its parts are even.

Given a partition $\lambda$ of length at most $n$, the Schur function in $n$ variables associated to $\lambda$ is a certain symmetric polynomial $s_{\lambda}=s_{\lambda}\left(x_{1}, \ldots, x_{n}\right)$ with integral coefficients. We do not need the precise definition here; see [34], p. 40. What we need to know is that $s_{\lambda}$ is exactly the character $\chi_{\mathrm{GL}(n)}(\lambda)$ of the finite-dimensional representation of $\mathrm{GL}_{n}(\mathbb{C})$ with highest weight $\lambda$, restricted to the maximal torus. Then we find in 
$[34]$, p. 76 , the formula

$$
\sum_{\lambda} s_{\lambda}\left(x_{1}, \ldots, x_{n}\right)=\prod_{i=1}^{n}\left(1-x_{i}\right)^{-1} \prod_{1 \leq i<j \leq n}\left(1-x_{i} x_{j}\right)^{-1},
$$

where the sum is over partitions $\lambda$ of length at most $n$. So if $\Pi$ is an irreducible admissible spherical representation of $\mathrm{GL}_{n}(F)$ with Satake parameter $z_{\Pi}=\operatorname{diag}\left(z_{1}, \ldots, z_{n}\right)$, then since $s_{\lambda}$ is homogeneous of weight $|\lambda|$,

$$
L\left(s, \Pi, \rho_{\mathrm{TT}}^{\prime}\right)=\sum_{\lambda: \ell(\lambda) \leq n} \chi_{\mathrm{GL}(n)}(\lambda)\left(z_{\Pi}\right) q^{-s|\lambda|} .
$$

So we need to know how the characters $\chi_{\mathrm{GL}(4)}$ decompose when restricted to $\mathrm{Sp}_{4}(\mathbb{C})$. For this, we have a result of Koike and Terada [28]. First, some more definitions. A Young diagram is a (finite) collection of boxes arranged in left-justified rows, with the lengths of the rows weakly decreasing top to bottom. The list of the row lengths of such a diagram forms a partition, say $\lambda$, and the diagram is said to be of shape $\lambda$. Listing the column lengths of a Young diagram of shape $\lambda$ gives another partition denoted $\lambda^{\prime}$ and called the transpose of $\lambda$. Explicitly, $\lambda_{i}^{\prime}=\#\left\{j \mid \lambda_{j} \geq i\right\}$. For partitions $\lambda$ and $\mu$, we write $\mu \subseteq \lambda$ when $\mu_{i} \leq \lambda_{i}$ for all $i$, and $\lambda+\mu$ is the partition $\left(\lambda_{1}+\right.$ $\left.\mu_{1}, \lambda_{2}+\mu_{2}, \ldots\right)$.

A Young tableau (or simply tableau) is a filling of a Young diagram with the symbols $\{1,2, \ldots, n\}$ (with the usual ordering), where $n \leq|\lambda|$ and $\lambda$ is the shape of the diagram. A tableau is called column-strict (or semistandard) if the entries are weakly increasing along the rows and strictly increasing along the columns; a semistandard tableau which has entries strictly increasing along rows is called standard. The weight of a tableau is the sequence which records the number of times each entry appears in the tableau. Thus, for example, all standard tableau have weight $(1,1, \ldots, 1)$. 


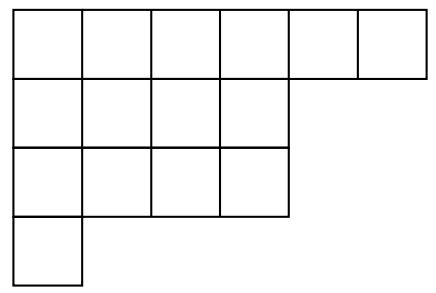

Figure 3.1: A Young diagram of shape $(6,4,4,1)$

\begin{tabular}{|l|l|l|l|l|l|}
\hline 1 & 2 & 2 & 4 & 5 & 8 \\
\cline { 1 - 2 } 3 & 5 & 5 & 5 & \multicolumn{2}{|c}{} \\
\cline { 1 - 2 } 6 & 6 & 7 & 8 & \multicolumn{2}{|c}{} \\
\cline { 1 - 2 } 8 & \multicolumn{4}{|l}{} \\
\cline { 1 - 2 } & & \multicolumn{2}{|l}{}
\end{tabular}

Figure 3.2: A column-strict Young tableau

Given a pair of partitions $\lambda, \mu$ with $\lambda \supseteq \mu$, the skew diagram of skew-shape $\lambda / \mu$ is obtained by removing the diagram of shape $\mu$ from the diagram of shape $\lambda$. We have the analogous notions of skew tableau and semistandard and standard skew tableau. The word of a skew tableu is the string obtained by listing the entries of the tableau from right to left and top to bottom. We may also define the weight of a word to be the sequence giving the number of times each symbol appears in the word, with 


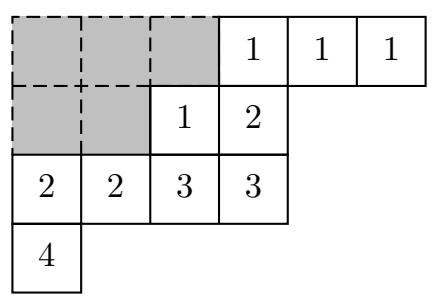

Figure 3.3: Skew tableau of skew-shape $(6,4,4,1) /(3,2)$

entries in increasing order. A word in $\{1,2, \ldots, n\}$ is called a lattice permutation if, in any initial segment of the word, the symbol $i$ appears at least as often as the symbol $i+1$. So, for example, the word of the tableau in Figure 3.3 is 1112133224 , and this is not a lattice permutation since the initial segment 1112133 has more 3's than 2's. Note that a word is a lattice permutation if and only if the weight of every initial segment is a partition.

For a partition $\mu$ with $\ell(\mu) \leq n$, let $V^{\mu}$ be the finite-dimensional representation of $\mathrm{GL}_{n}(\mathbb{C})$ of highest weight $\mu$. Then the Littlewood-Richardson coefficients $c_{\mu, \beta}^{\lambda}$ are defined by

$$
V^{\mu} \otimes V^{\beta}=\sum_{\lambda} c_{\mu, \beta}^{\lambda} V^{\lambda}
$$

Note that this implies $c_{\mu, \beta}^{\lambda}=c_{\beta, \mu}^{\lambda}$. The Littlewood-Richardson rule ([34], p. 143) gives an alternate characterization of these coefficients in terms of tableax, namely

For partitions $\lambda, \mu, \beta$, the coefficient $c_{\mu, \beta}^{\lambda}$ is the number of column-strict 
tableaux of skew-shape $\lambda / \mu$ and weight $\beta$ whose word is a lattice permutation.

From this characterization, we see that $c_{\mu, \beta}^{\lambda}=0$ unless $\lambda \supseteq \mu, \lambda \supseteq \beta$ and $|\lambda|=|\mu|+|\beta|$. Now we may state the result of Koike-Terada.

Theorem 3.1.11 ([28], p. 504-506). Let $\lambda$ be a partition of length at most $2 n$. Then

$$
\operatorname{Res}_{\mathrm{Sp}(2 n)}^{\mathrm{GL}(2 n)} \chi_{\mathrm{GL}(2 n)}(\lambda)=\sum_{\mu} \sum_{\beta^{\prime} \text { even }} c_{\mu, \beta}^{\lambda} \tilde{\chi}(\mu)
$$

where the sum is over all $\mu$ and $\beta$ (with $\beta^{\prime}$ even), $\tilde{\chi}(\mu)$ is 0 or $\pm_{\mathrm{Sp}(2 n)}(\tilde{\mu}), \tilde{\mu}$ is a partition derived from $\mu$, and both are determined as follows. Set $\mu^{\prime}=\left(\mu_{1}^{\prime}, \ldots, \mu_{l}^{\prime}\right)$.

(i). If $\mu_{i}^{\prime}-i+\mu_{j}^{\prime}-j=2 n$ for any $1 \leq i, j \leq n$, then $\tilde{\chi}(\mu)=0$.

(ii). Otherwise, set

$$
k_{i}= \begin{cases}\mu_{i}^{\prime} & \text { if } \mu_{i}^{\prime}-i<n ; \\ 2 n+2 i-\mu_{i}^{\prime} & \text { if } \mu_{i}^{\prime}-i>n .\end{cases}
$$

Let $s$ be the number of indices $i$ such that $\mu_{i}^{\prime}-i>n$, and let $t_{i}=k_{i}-(i-1)$. By hypothesis, the $t_{i}$ are distinct, so there is a permutation $\sigma$ such that $t_{\sigma(1)}>$ $t_{\sigma(2)}>\cdots>t_{\sigma(l)}$. Set $\tilde{\mu}_{i}^{\prime}=t_{\sigma(i)}+(i-1)=k_{\sigma(i)}+i-\sigma(i)$. Define $\tilde{\mu}$ to be the partition whose transpose is $\tilde{\mu}^{\prime}=\left(\tilde{\mu}_{1}^{\prime}, \ldots, \tilde{\mu}_{l}^{\prime}\right)$. Then we have

$$
\tilde{\chi}(\mu)=(-1)^{s} \operatorname{sgn} \sigma \chi_{\operatorname{Sp}(2 n)}(\tilde{\mu}) .
$$

In particular, $\tilde{\chi}(\mu)=\chi_{\mathrm{Sp}(2 n)}(\mu)$ if $\ell(\mu) \leq n$. 
In our case $n=2$, so all partitions appearing in (3.18) with nonzero coefficients will have length at most 4 . Applying the above to $\mu=\left(\mu_{1}, \mu_{2}, \mu_{3}, \mu_{4}\right)$ shows that

$$
\tilde{\chi}(\mu)= \begin{cases}\chi_{\mathrm{Sp}(4)}\left(\left(\mu_{1}, \mu_{2}\right)\right) & \text { if } \mu_{3}=\mu_{4}=0 ; \\ -\chi_{\mathrm{Sp}(4)}\left(\left(\mu_{1}, \mu_{2}\right)\right) & \text { if } \mu_{3}=\mu_{4}=1 ; \\ 0 & \text { otherwise. }\end{cases}
$$

Hence we may rewrite (3.18) as

$$
\operatorname{Res}_{\mathrm{Sp}(4)}^{\mathrm{GL}(4)} \chi_{\mathrm{GL}(4)}(\lambda)=\sum_{\mu: \ell(\mu) \leq 2} \chi_{\mathrm{Sp}(4)}(\mu) \sum_{\beta^{\prime} \text { even }}\left(c_{\mu, \beta}^{\lambda}-c_{\mu^{+}, \beta}^{\lambda}\right),
$$

where $\mu^{+}=\mu+(0,0,1,1)=\left(\mu_{1}, \mu_{2}, 1,1\right)$.

So we need to understand the coefficients $c_{\mu, \beta}^{\lambda}$ and their sums over $\beta$ with $\beta^{\prime}$ even.

Proposition 3.1.12. Let $\lambda, \mu, \beta$ be partitions such that $\lambda \supseteq \mu, \lambda \supseteq \beta,|\lambda|=|\mu|+$ $|\beta|, \ell(\lambda) \leq 4$ and $\beta^{\prime}$ is even (i.e., $\beta_{1}=\beta_{2}$ and $\beta_{3}=\beta_{4}$ ). Set $L_{i}=\mu_{i}+\beta_{i}-\lambda_{i}$, $1 \leq i \leq 4$, so that $\sum L_{i}=0$.

(i). If $\mu_{i}=\mu_{j}$ or if $\lambda_{i}=\lambda_{j}$ for any $i \neq j$, then $c_{\mu, \beta}^{\lambda} \leq 1$.

(ii). Suppose $\mu_{3}=\mu_{4}$. Then $c_{\mu, \beta}^{\lambda}=1$ if and only if all of the following hold:

$$
\begin{aligned}
& \text { (a). } 0 \leq-L_{4} \leq L_{2} \leq \mu_{2}-\mu_{3} ; \\
& \text { (b). } 0 \leq L_{1}+L_{4} \leq \mu_{1}-\mu_{2} ; \\
& \text { (c). }-L_{3} \leq \beta_{2}-\beta_{4} .
\end{aligned}
$$

This will be proven in Section 3.3. As for the sums of these coefficients, the following result gives what we need. 
Proposition 3.1.13. Given a nonnegative integer $M$ and a partition $\mu=\left(\mu_{1}, \mu_{2}\right)$ of length at most two, define

$$
B_{\mu, M}=\sum_{\substack{\lambda \nmid M \\ \ell(\lambda) \leq 4}} \sum_{\beta^{\prime} \text { even }}\left(c_{\mu, \beta}^{\lambda}-c_{\mu^{+}, \beta}^{\lambda}\right)
$$

where $\mu^{+}=\mu+(0,0,1,1)=\left(\mu_{1}, \mu_{2}, 1,1\right)$. Then we have

$$
\sum_{N=0}^{\infty} B_{\mu,|\mu|+2 N} X^{N}=\frac{\left(1-X^{1+\mu_{2}}\right)\left(1-X^{1+\mu_{1}-\mu_{2}}\right)}{(1-X)^{3}\left(1-X^{2}\right)}
$$

as functions in $X$.

This will also be proved in Section 3.3. Note also that

$$
B_{\mu, M}=0 \text { for } M \leq|\mu| \text { or if } M-|\mu| \text { is odd, }
$$

since $B_{\mu, M}$ involves only those $\beta$ with $\beta^{\prime}$ even, and since $c_{\mu, \beta}^{\lambda}=0$ unless $|\lambda|-|\mu|=$ $M-|\mu|=|\beta|$, which is certainly even if $\beta^{\prime}$ is. With these facts in hand, we can now prove

Theorem 3.1.14. Let $s^{\prime}=(s+1) / 2$. Then

$$
\Psi^{*}\left(s, W^{\circ}, f_{s}^{\circ}\right)=L\left(s^{\prime}, \pi, \rho_{\mathrm{TT}}\right) .
$$

Proof. In the notation of Theorem 3.1.10, $\operatorname{tr} \tau_{(k, l)}$ is the character of the finitedimensional representation of $\mathrm{Sp}_{4}(\mathbb{C})$ of highest weight $k \omega_{1}+l\left(\omega_{1}+\omega_{2}\right)=(k+l) \omega_{1}+l \omega_{2}$ and so corresponds to the partition $\mu=(k+l, l)$. Thus if $z_{\pi}$ is the Satake parameter 
for $\pi$, thought of as a semisimple element in $\mathrm{Sp}_{4}(\mathbb{C})$ ( since $\rho_{\mathrm{TT}}$ is trivial on similitudes), $\operatorname{tr} \tau_{(k, l)}\left(z_{\pi}\right)=\chi_{\mathrm{Sp}(4)}((k+l, l))\left(z_{\pi}\right)=\chi_{\mathrm{Sp}(4)}((k+l, l))$ (we will often supress the $z_{\pi}$ for brevity). Hence (3.10) becomes

$$
\begin{aligned}
\Psi^{*}\left(s, W^{\circ}, f_{s}^{\circ}\right) & =\sum_{k, l \geq 0} \chi_{\operatorname{Sp}(4)}((k+l, l)) X^{k+2 l} \frac{\left(1-X^{2(k+1)}\right)\left(1-X^{2(l+1)}\right)}{\left(1-X^{2}\right)^{3}\left(1-X^{4}\right)} \\
& =\sum_{\mu: \ell(\mu) \leq 2} \chi_{\operatorname{Sp}(4)}(\mu) X^{|\mu|} \frac{\left(1-X^{2\left(1+\mu_{1}-\mu_{2}\right)}\right)\left(1-X^{2\left(1+\mu_{2}\right)}\right)}{\left(1-X^{2}\right)^{3}\left(1-X^{4}\right)} \\
& =\sum_{\mu: \ell(\mu) \leq 2} \chi_{\operatorname{Sp}(4)}(\mu) X^{|\mu|}\left(\sum_{N=0}^{\infty} B_{\mu,|\mu|+2 N} X^{2 N}\right) .
\end{aligned}
$$

Now the inner sum is, by (3.24),

$$
\begin{aligned}
\sum_{N=0}^{\infty} & B_{\mu,|\mu|+2 N} X^{2 N}=\sum_{N=0}^{\infty} B_{\mu,|\mu|+N} X^{N}=\sum_{N=|\mu|}^{\infty} B_{\mu, N} X^{N-|\mu|} \\
& =\sum_{N=|\mu|}^{\infty} \sum_{\substack{\lambda \nmid N \\
\ell(\lambda) \leq 4}} \sum_{\beta^{\prime} \text { even }}\left(c_{\mu, \beta}^{\lambda}-c_{\mu^{+}, \beta}^{\lambda}\right) X^{|\lambda|-|\mu|} \\
& =\sum_{\substack{\lambda: \lambda \supseteq \mu \\
\ell(\lambda) \leq 4}} \sum_{\beta^{\prime} \text { even }}\left(c_{\mu, \beta}^{\lambda}-c_{\mu^{+}, \beta}^{\lambda}\right) X^{|\lambda|-|\mu|},
\end{aligned}
$$

so substituting into the above and exchanging summation of $\lambda$ and $\mu$ gives

$$
\begin{aligned}
\Psi^{*}\left(s, W^{\circ}, f_{s}^{\circ}\right)=\sum_{\mu: \ell(\mu) \leq 2} \chi_{\operatorname{Sp}(4)}(\mu) X^{|\mu|} \sum_{\substack{\lambda: \lambda \supset \mu \\
\ell(\lambda) \leq 4}} \sum_{\beta^{\prime} \text { even }}\left(c_{\mu, \beta}^{\lambda}-c_{\mu^{+}, \beta}^{\lambda}\right) X^{|\lambda|-|\mu|} \\
=\sum_{\lambda: \ell(\lambda) \leq 4} X^{|\lambda|} \sum_{\substack{\mu: \mu \subseteq \lambda \\
\ell(\mu) \leq 2}} \chi_{\operatorname{Sp}(4)}(\mu)\left(z_{\pi}\right) \sum_{\beta^{\prime} \text { even }}\left(c_{\mu, \beta}^{\lambda}-c_{\mu^{+}, \beta}^{\lambda}\right) \\
=\sum_{\lambda: \ell(\lambda) \leq 4} \chi_{\operatorname{GL}(4)}(\lambda)\left(z_{\pi}\right) X^{|\lambda|}
\end{aligned}
$$


the last equality following from (3.21). Recall $X=q^{-s^{\prime}}$ and $s^{\prime}=(s+1) / 2$. Therefore the last line is just

$$
\sum_{\lambda: \ell(\lambda) \leq 4} \chi_{\mathrm{GL}(4)}(\lambda)\left(z_{\pi}\right) q^{-s^{\prime}|\lambda|}=L\left(s^{\prime}, \pi, \rho_{\mathrm{TT}}\right)
$$

as we wanted.

\subsection{Split case}

Now suppose $v$ is split in $E$, so we have $E_{v} \simeq F_{v} \oplus F_{v}$. We show two things here: first, that our integral agrees with the local integral found in [24]; second, that this integral actually calculates the correct $L$-factor. As mentioned above, Jiang accomplishes this in his thesis by using the Kostant-Rallis algorithm. We give a new proof using the branching laws stated in the previous section. We wish to thank Professor Jiang for the suggestion to carry out this computation.

In order to clarify what follows, we make the above isomorphism explicit. Since $v$ splits in $E$, we have

$$
E_{v} \stackrel{\text { def }}{=} F_{v} \otimes_{F} E \stackrel{r}{\sim} F_{v} \oplus F_{v} .
$$

Since $E_{v}$ is spanned by the elements $a \otimes 1$ for $a \in F_{v}$ and $1 \otimes \theta$, the isomorphism $r$ can be described by

$$
r:\left\{\begin{array}{l}
a \otimes 1 \mapsto(a, a), \quad a \in F_{v} \\
1 \otimes \theta \mapsto\left(\theta_{v},-\theta_{v}\right),
\end{array}\right.
$$


since $\theta_{v} \in F$. If we denote the usual conjugation mappings by $\alpha \mapsto \bar{\alpha}$, i.e.,

$$
\begin{array}{ll}
\alpha=a+b \theta \mapsto \bar{\alpha}=a-b \theta, & \alpha \in E_{v}, \\
\beta=(a, b) \mapsto \bar{\beta}=(b, a), & \beta \in F_{v} \oplus F_{v},
\end{array}
$$

then the isomorphism $r$ is compatible with these conjugations:

$$
\begin{aligned}
\overline{r(a+b \theta)} & =\overline{\left(a+b \theta_{v}, a-b \theta_{v}\right)}=\left(a-b \theta_{v}, a+b \theta_{v}\right) \\
& =r(a-b \theta)=r(\overline{a+b \theta}) .
\end{aligned}
$$

So we may drop the subscript $v$ and unambiguously refer to "conjugation with respect to $\theta$," bearing in mind that under $E \simeq F \oplus F$ we have $\theta \leftrightarrow(\theta,-\theta)$.

\subsubsection{The Split Integral}

In this subsection only, we allow $F$ to be archimedean as well, so we consider the cases $F$ nonarchimedean, $F=\mathbb{R}$ and $F=\mathbb{C}$. We will treat all cases simultaneously and note any additional arguments as they are needed.

Since $E / F$ is split, $\operatorname{GSp}_{4}(E) \simeq \operatorname{GSp}_{4}(F) \times \operatorname{GSp}_{4}(F)$. More explicitly, consider the commutative diagram

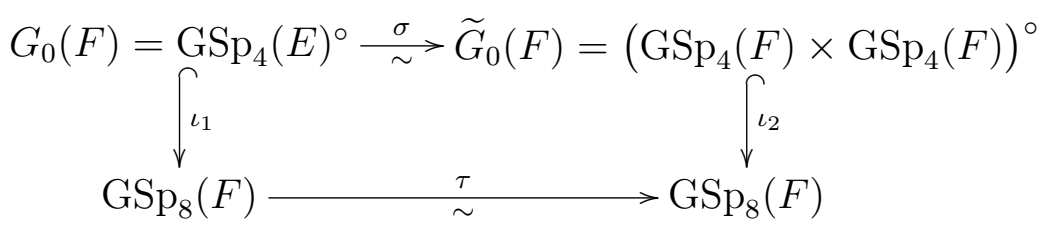

where

$$
\left(\operatorname{GSp}_{4}(F) \times \operatorname{GSp}_{4}(F)\right)^{\circ}=\left\{\left(g_{1}, g_{2}\right) \mid g_{i} \in \operatorname{GSp}_{4}(F), \lambda\left(g_{1}\right)=\lambda\left(g_{2}\right)\right\}
$$


and $\iota_{1}$ is defined by $(2.2), \iota_{2}$ is the map

$$
\iota_{2}\left(\left(\begin{array}{ll}
A & B \\
C & D
\end{array}\right),\left(\begin{array}{cc}
A^{\prime} & B^{\prime} \\
C^{\prime} & D^{\prime}
\end{array}\right)\right)=\left(\begin{array}{cccc}
A & & B & \\
& A^{\prime} & & -B^{\prime} \\
C & -C^{\prime} & D & D^{\prime}
\end{array}\right)
$$

and $\sigma$ and $\tau$ are isomorphisms defined by

$$
\sigma:\left\{\begin{array}{l}
\operatorname{GSp}_{4}(E) \rightarrow \mathrm{GSp}_{4}(F) \times \mathrm{GSp}_{4}(F) \\
g \mapsto\left(g,\left({ }^{I_{2}}-I_{2}\right) \bar{g}\left({ }^{I_{2}}-I_{2}\right)\right)
\end{array}\right.
$$

and

$$
\tau(h)=C_{\theta}^{-1} h C_{\theta},
$$

where

$$
\begin{gathered}
C_{\theta}=\left(\begin{array}{ll}
Y_{\theta} & \\
& T_{\theta}^{-1} Y_{\theta}
\end{array}\right), \\
Y_{\theta}=\left(\begin{array}{ccc}
1 & 1 & \\
\theta & -1 & \\
1 & & -1 \\
\theta & -\theta
\end{array}\right), \quad T_{\theta}=\operatorname{diag}\left(1, \theta^{2}, 1, \theta^{2}\right)
\end{gathered}
$$

and $g \mapsto \bar{g}$ is conjugation with respect to $\theta$. Note that since $\theta$ is a unit, $C_{\theta} \in \operatorname{GSp}_{8}(\mathcal{O})$. (If $F$ is archimedean, it is clear that $C_{\theta} \in \mathrm{O}_{8}(F)$.) It is easy to check that

$$
\left(\tau \circ \iota_{1}\right)(g)=\left(\iota_{2} \circ \sigma\right)(g), \quad \text { for } g \in G_{0}(F) \text {. }
$$

Now $\pi$ is an irreducible admissible representation of $\operatorname{GSp}_{4}(E)$, so it decomposes under $\sigma$ as $\pi \simeq \pi \circ \sigma^{-1}=\pi_{1} \otimes \pi_{2}$, where $\pi_{1}, \pi_{2}$ are irreducible admissible representations of $\operatorname{GSp}_{4}(F)$, and if $\pi$ is spherical, $\pi_{1}, \pi_{2}$ will be as well. Let $W_{1}^{\circ}, W_{2}^{\circ}$ be the spherical 
Whittaker functions for $\pi_{1}, \pi_{2}$ respectively, normalized so that $W_{1}^{\circ}(1)=1=W_{2}^{\circ}(1)$. Then we have a similar decomposition for the spherical Whittaker function $W^{\circ}$ of $\pi$ :

$$
W^{\circ}\left(\sigma^{-1}\left(g_{1}, g_{2}\right)\right)=W_{1}^{\circ}\left(g_{1}\right) W_{2}^{\circ}\left(g_{2}\right) \text {. }
$$

Jiang's [24, I.2.2.1] local, unramified integral is

$$
\widetilde{\Psi}\left(s, W_{1}^{\circ}, W_{2}^{\circ}, f_{s}^{\circ}\right)=\int_{\widetilde{\mathcal{D}}(F)} f_{s}^{\circ}\left(\tilde{\gamma}_{0} \iota_{2}\left(g_{1}, g_{2}\right)\right) W_{1}^{\circ}\left(g_{1}\right) W_{2}^{\circ}\left(g_{2}\right) d\left(g_{1}, g_{2}\right)
$$

where

$$
\begin{aligned}
\widetilde{\mathcal{D}}(F)= & Z(F) N^{P^{\Delta}}(F)\left(X_{2}^{P_{4}}(F) \times I_{4}\right) \backslash \widetilde{G}_{0}(F), \\
& P_{4}^{\Delta}(F)=\left\{(g, g) \mid g \in P_{4}(F)\right\},
\end{aligned}
$$

and

$$
\tilde{\gamma}_{0}=\left(\begin{array}{cccccccc}
1 & & & & 0 & & \\
& 1 & & & 0 & \\
& & 0 & 0 & & 0 & 1 \\
0 & & & 1 & 1 & 0 \\
0 & & & & 1 & 1 \\
& 1 & & -1 & & 0 & 1 \\
1 & & -1 & & & & 0
\end{array}\right) .
$$

Recall our local integral is

$$
\Psi\left(s, W^{\circ}, f_{s}^{\circ}\right)=\int_{\mathcal{D}(F)} f_{s}\left(\gamma_{0} \iota_{1}(g)\right) W^{\circ}(g) d g
$$

where

$$
\mathcal{D}(F)=Z(F) N^{Q}(F) X_{2}^{Q^{\prime}}(F) \backslash G_{0}(F)
$$

and $\gamma_{0}$ is given by (2.9). To see this integral is the same as (3.27), we first note that $Q(F) \subseteq\left\{\left(\begin{array}{cc}A & B \theta \\ C \theta & D\end{array}\right)\right\}$, so that

$$
\sigma(Q(F)) \subseteq\left\{\left(\left(\begin{array}{cc}
A & B \theta \\
C \theta & D
\end{array}\right),\left(\begin{array}{cc}
A & B \theta \\
C \theta & D
\end{array}\right)\right)\right\}
$$


Since we clearly have $\sigma(Q(F)) \subseteq P_{4}(F) \times P_{4}(F)$, it follows that

$$
\sigma(Q(F))=P_{4}^{\Delta}(F) \Rightarrow \sigma\left(N^{Q}(F)\right)=N^{P_{4}{ }^{\Delta}}(F) .
$$

It is equally clear that $\sigma\left(X_{2}^{Q}(F)\right)=X_{2}^{P_{4}{ }^{\Delta}}(F) \stackrel{\text { def }}{=}\left\{(w, w) \mid w \in X_{2}^{P_{4}}(F)\right\}$. For $\alpha \in E$, let

$$
w(\alpha)=\chi_{2 \epsilon_{2}}(\alpha)=\left(\begin{array}{cccc}
1 & & & \\
& 1 & & \\
& & 1 & \\
& & & 1
\end{array}\right) \in X_{2}^{P_{4}}(E)
$$

then we may write

$$
\begin{aligned}
\sigma(w(\alpha)) & =(w(\alpha), w(-\bar{\alpha})) \\
& =(w(-\bar{\alpha}), w(-\bar{\alpha})) \cdot(w(\alpha+\bar{\alpha}), 1) \\
& \in X_{2}^{P_{4} \Delta}(F) \cdot\left(X_{2}^{P_{4}}(F) \times I_{4}\right),
\end{aligned}
$$

so that

$$
\sigma\left(X_{2}^{P_{4}}(E)\right)=X_{2}^{P_{4}{ }^{\Delta}}(F) \cdot\left(X_{2}^{P_{4}}(F) \times I_{4}\right)
$$

and

$$
\sigma\left(N^{Q}(F) X_{2}^{Q^{\prime}}(F)\right)=N^{P_{4}{ }^{\Delta}}(F) \cdot\left(X_{2}^{P_{4}}(F) \times I_{4}\right)
$$

which gives

$$
\sigma(\mathcal{D}(F))=\widetilde{\mathcal{D}}(F)
$$

Also note that $\gamma_{0} C_{\theta}=p_{\theta} \tilde{\gamma}_{0}$, where

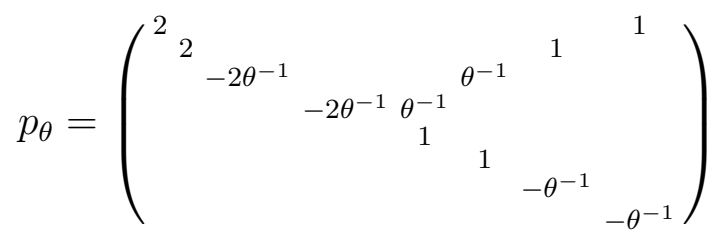


is in $P_{8}(F)$, and $\delta_{P_{8}}\left(p_{\theta}\right)=2^{9} \theta^{-6}$ is a unit since $\theta$ is a unit and the residue characteristic of $F$ is not 2 . Hence $f_{s}^{\circ}\left(p_{\theta}\right)=1$.

Finally, we normalize the measures on $\mathcal{D}(F)$ and $\widetilde{\mathcal{D}}(F)$ compatibly:

$$
\int_{\mathcal{D}(F)} \phi(g) d g=\int_{\widetilde{\mathcal{D}}(F)} \phi\left(\sigma^{-1}\left(g_{1}, g_{2}\right)\right) d\left(g_{1}, g_{2}\right)
$$

for absolutely integrable functions $\phi$ on $\mathcal{D}(F)$.

Proposition 3.2.1. Notation as above, we have

$$
\Psi\left(s, W^{\circ}, f_{s}^{\circ}\right)=\widetilde{\Psi}\left(s, W_{1}^{\circ}, W_{2}^{\circ}, f_{s}^{\circ}\right) .
$$

Proof.

$$
\begin{aligned}
\Psi\left(s, W^{\circ}, f_{s}^{\circ}\right) & =\int_{\mathcal{D}(F)} f_{s}^{\circ}\left(\gamma_{0} \iota_{1}(g)\right) W^{\circ}(g) d g \\
& =\int_{\sigma(\mathcal{D}(F))} f_{s}^{\circ}\left(\gamma_{0} \iota_{1} \circ \sigma^{-1}\left(g_{1}, g_{2}\right)\right) W^{\circ}\left(\sigma^{-1}\left(g_{1}, g_{2}\right)\right) d\left(g_{1}, g_{2}\right) \\
& =\int_{\widetilde{\mathcal{D}}(F)} f_{s}^{\circ}\left(\gamma_{0} \tau^{-1} \circ \iota_{2}\left(g_{1}, g_{2}\right)\right) W_{1}^{\circ}\left(g_{1}\right) W_{2}^{\circ}\left(g_{2}\right) d\left(g_{1}, g_{2}\right) \\
& =\int_{\widetilde{\mathcal{D}}(F)} f_{s}^{\circ}\left(\gamma_{0} C_{\theta} \iota_{2}\left(g_{1}, g_{2}\right) C_{\theta}^{-1}\right) W_{1}^{\circ}\left(g_{1}\right) W_{2}^{\circ}\left(g_{2}\right) d\left(g_{1}, g_{2}\right) \\
& =\int_{\widetilde{\mathcal{D}}(F)} f_{s}^{\circ}\left(p_{\theta} \tilde{\gamma}_{0} \iota_{2}\left(g_{1}, g_{2}\right)\right) W_{1}^{\circ}\left(g_{1}\right) W_{2}^{\circ}\left(g_{2}\right) d\left(g_{1}, g_{2}\right) \\
& =\int_{\widetilde{\mathcal{D}}(F)} f_{s}^{\circ}\left(\tilde{\gamma}_{0} \iota_{2}\left(g_{1}, g_{2}\right)\right) W_{1}^{\circ}\left(g_{1}\right) W_{2}^{\circ}\left(g_{2}\right) d\left(g_{1}, g_{2}\right) \\
& =\widetilde{\Psi}\left(s, W_{1}^{\circ}, W_{2}^{\circ}, f_{s}^{\circ}\right) .
\end{aligned}
$$

Jiang calculates the integral in [24]. 
Theorem 3.2.2 ([24], I.3.3.2). Let $X=q^{-\frac{s+1}{2}}$. Then the normalized unramified [split] local integral is

$$
\begin{aligned}
\Psi^{*}(s, & \left.W_{1}^{\circ}, W_{2}^{\circ}, f_{s}^{\circ}\right)=d_{G, v}(s) \Psi\left(s, W_{1}^{\circ}, W_{2}^{\circ}, f_{s}^{\circ}\right) \\
= & \frac{1}{\left(1-X^{2}\right)^{3}\left(1-X^{4}\right)} \\
& \cdot\left\{\sum_{\substack{k_{1}+k_{2} \geq l_{1}+l_{2} \geq k_{1} \\
2 l_{1} \geq k_{1} \geq l_{1}}} \operatorname{tr} \tau_{\left(k_{1}, k_{2} \mid 2 l_{1}-k_{1}, l_{2}\right)}\left(x_{\pi_{1}}, y_{\pi_{2}}\right) P_{1}\left(k_{1}, k_{2}, l_{1}, l_{2} ; X\right)\right. \\
& +\sum_{\substack{k_{1}+k_{2} \geq l_{1}+l_{2} \\
l_{1}>k_{1}}} \operatorname{tr} \tau_{\left(k_{1}, k_{2} \mid 2 l_{1}-k_{1}, l_{2}\right)}\left(x_{\pi_{1}}, y_{\pi_{2}}\right) P_{2}\left(k_{1}, k_{2}, l_{1}, l_{2} ; X\right) \\
& +\sum_{\substack{k_{1}+k_{2}<l_{1}+l_{2} \\
2 l_{1} \geq k_{1} \geq l_{1}}} \operatorname{tr} \tau_{\left(k_{1}, k_{2} \mid 2 l_{1}-k_{1}, l_{2}\right)}\left(x_{\pi_{1}}, y_{\pi_{2}}\right) P_{3}\left(k_{1}, k_{2}, l_{1}, l_{2} ; X\right) \\
& \left.+\sum_{\substack{l_{1} \leq k_{1}+k_{2}<l_{1}+l_{2} \\
l_{1}>k_{1}}} \operatorname{tr} \tau_{\left(k_{1}, k_{2} \mid 2 l_{1}-k_{1}, l_{2}\right)}\left(x_{\pi_{1}}, y_{\pi_{2}}\right) P_{4}\left(k_{1}, k_{2}, l_{1}, l_{2} ; X\right)\right\},
\end{aligned}
$$

where $d_{G, v}(s)=\zeta_{v}(2 s+2) \zeta_{v}(s+1) \zeta_{v}(s+2) \zeta_{v}(s+3)$ is the local component of the normalizing factor for the Eisenstein series, $\operatorname{tr} \tau_{\left(a_{1}, a_{2} \mid b_{1}, b_{2}\right)}\left(x_{\pi_{1}}, y_{\pi_{2}}\right)$ is the character of the tensor product of the finite-dimensional representations of $\mathrm{Sp}_{4}(\mathbb{C})$ of highest weights $a_{1} \omega_{1}+a_{2}\left(\omega_{1}+\omega_{2}\right)$ and $b_{1} \omega_{1}+b_{2}\left(\omega_{1}+\omega_{2}\right)$, evaluated at the Satake parameter $\left(x_{\pi_{1}}, y_{\pi_{2}}\right) \in\left(\mathrm{GSp}_{4}(\mathbb{C}) \times \mathrm{GSp}_{4}(\mathbb{C})\right)^{\circ}$ for $\pi_{1} \otimes \pi_{2}$, and

$$
\begin{aligned}
& P_{1}\left(k_{1}, k_{2}, l_{1}, l_{2}, X\right)=X^{3 k_{1}+2 k_{2}-2 l_{1}}\left(1-X^{2\left(1+l_{1}+l_{2}-k_{1}\right)}\right)\left(1-X^{2\left(1+2 l_{1}-k_{1}\right)}\right) \\
& P_{2}\left(k_{1}, k_{2}, l_{1}, l_{2}, X\right)=X^{2 k_{2}-k_{1}+2 l_{1}}\left(1-X^{2\left(1+l_{2}\right)}\right)\left(1-X^{2\left(1+k_{1}\right)}\right) \\
& P_{3}\left(k_{1}, k_{2}, l_{1}, l_{2}, X\right)=X^{k_{1}+2 l_{2}}\left(1-X^{2\left(1+k_{2}\right)}\right)\left(1-X^{2\left(1+2 l_{1}-k_{1}\right)}\right) \\
& P_{4}\left(k_{1}, k_{2}, l_{1}, l_{2}, X\right)=X^{4 l_{1}+2 l_{2}-3 k_{1}}\left(1-X^{2\left(1+k_{1}+k_{2}-l_{1}\right)}\right)\left(1-X^{2\left(1+k_{1}\right)}\right) .
\end{aligned}
$$


It will be useful in the next section to rephrase (3.33) in terms of partitions. With all notation as in the previous theorem, set

$$
\mu=\left(\mu_{1}, \mu_{2}\right)=\left(k_{1}+k_{2}, k_{2}\right), \quad \nu=\left(\nu_{1}, \nu_{2}\right)=\left(2 l_{1}-k_{1}+l_{2}, l_{2}\right),
$$

so that $\mu$ and $\nu$ are partitions, provided $2 l_{1} \geq k_{1}$. Let $\chi_{\operatorname{Sp}(4)}(\mu)$ be the character of the finite-dimensional representation of $\operatorname{Sp}_{4}(\mathbb{C})$ with highest weight $\mu_{1} \omega_{1}+\mu_{2} \omega_{2}=$ $k_{1} \omega_{1}+k_{2}\left(\omega_{1}+\omega_{2}\right)$, and similarly for $\chi_{\operatorname{Sp}(4)}(\nu)$. So if the Satake parameter for $\pi_{1} \otimes \pi_{2}$ is represented by the semisimple element $\left(x_{\pi_{1}}, y_{\pi_{2}}\right) \in\left(\operatorname{GSp}_{4}(\mathbb{C}) \times \operatorname{GSp}_{4}(\mathbb{C})\right)^{\circ}$, then

$$
\operatorname{tr} \tau_{\left(k_{1}, k_{2} \mid 2 l_{1}-k_{1}, l_{2}\right)}\left(x_{\pi_{1}}, y_{\pi_{2}}\right)=\chi_{\operatorname{Sp}(4)}(\mu)\left(x_{\pi_{1}}\right) \chi_{\operatorname{Sp}(4)}(\nu)\left(y_{\pi_{2}}\right) .
$$

Define $\delta_{\mu}=\mu_{1}-\mu_{2}, \delta_{\nu}=\nu_{1}-\nu_{2}$ and

$$
\begin{array}{cc}
M_{1}=\max \{|\mu|,|\nu|\}, & M_{2}=\min \{|\mu|,|\nu|\}, \\
m_{1}=\max \left\{\delta_{\mu}, \delta_{\nu}\right\}, & m_{2}=\min \left\{\delta_{\mu}, \delta_{\nu}\right\} .
\end{array}
$$

Substituting $\mu, \nu$ into (3.33) yields

$$
\begin{aligned}
\Psi^{*}\left(s, W_{1}^{\circ}, W_{2}^{\circ}, f_{s}^{\circ}\right)=\frac{1}{\left(1-X^{2}\right)^{3}\left(1-X^{4}\right)} \\
\cdot \sum_{i=1}^{4} \sum_{\mu, \nu \in \mathcal{A}_{i}} \chi_{\operatorname{Sp}(4)}(\mu)\left(x_{\pi_{1}}\right) \chi_{\operatorname{Sp}(4)}(\nu)\left(y_{\pi_{2}}\right) P_{i}(\mu, \nu ; X),
\end{aligned}
$$

where

$$
\begin{aligned}
& \mathcal{A}_{1}=\left\{\mu, \nu \text { partitions }:|\mu| \geq|\nu| \geq \delta_{\mu} \geq \delta_{\nu}\right\} \\
& \mathcal{A}_{2}=\left\{\mu, \nu \text { partitions }:|\mu| \geq|\nu| \geq \delta_{\nu}>\delta_{\mu}\right\} \\
& \mathcal{A}_{3}=\left\{\mu, \nu \text { partitions }:|\nu|>|\mu| \geq \delta_{\mu} \geq \delta_{\nu}\right\} \\
& \mathcal{A}_{4}=\left\{\mu, \nu \text { partitions }:|\nu|>|\mu| \geq \delta_{\nu}>\delta_{\mu}\right\}
\end{aligned}
$$


(which are disjoint sets) and

$$
\begin{aligned}
& P_{1}(\mu, \nu ; X)=X^{|\mu|+\delta_{\mu}-\delta_{\nu}}\left(1-X^{2\left(1+\frac{1}{2}\left(|\nu|-\delta_{\mu}\right)\right)}\right)\left(1-X^{2\left(1+\delta_{\nu}\right)}\right) \\
& P_{2}(\mu, \nu ; X)=X^{|\mu|-\delta_{\mu}+\delta_{\nu}}\left(1-X^{2\left(1+\frac{1}{2}\left(|\nu|-\delta_{\nu}\right)\right)}\right)\left(1-X^{2\left(1+\delta_{\mu}\right)}\right) \\
& P_{3}(\mu, \nu ; X)=X^{|\nu|+\delta_{\mu}-\delta_{\nu}}\left(1-X^{2\left(1+\frac{1}{2}\left(|\mu|-\delta_{\mu}\right)\right)}\right)\left(1-X^{2\left(1+\delta_{\nu}\right)}\right) \\
& P_{4}(\mu, \nu ; X)=X^{|\nu|-\delta_{\mu}+\delta_{\nu}}\left(1-X^{2\left(1+\frac{1}{2}\left(|\mu|-\delta_{\nu}\right)\right)}\right)\left(1-X^{2\left(1+\delta_{\mu}\right)}\right) .
\end{aligned}
$$

So in terms of $M_{1}, M_{2}, m_{1}, m_{2}$, we have

$$
\Psi^{*}\left(s, W_{1}^{\circ}, W_{2}^{\circ}, f_{s}^{\circ}\right)=\sum_{\substack{\mu, \nu: M_{2} \geq m_{1} \\ \ell(\mu), \ell(\nu) \leq 2}} \chi_{\mathrm{Sp}(4)}(\mu)\left(x_{\pi_{1}}\right) \chi_{\mathrm{Sp}(4)}(\nu)\left(y_{\pi_{2}}\right) X^{M_{1}} P\left(\mu, \nu ; X^{2}\right),
$$

where

$$
P(\mu, \nu ; X)=\frac{X^{\frac{1}{2}\left(m_{1}-m_{2}\right)}\left(1-X^{1+\frac{1}{2}\left(M_{2}-m_{1}\right)}\right)\left(1-X^{1+m_{2}}\right)}{(1-X)^{3}\left(1-X^{2}\right)}
$$

\subsubsection{Local $L$-factor}

We now identify the integral (3.34) with the local (split, unramified) $L$-factor for $\pi$. The method is completely analogous to that used in the inert case.

Since $E / F$ is split, the $L$-group of $G_{0}$ is also split:

$$
{ }^{L} G_{0}=\left(\operatorname{GSp}_{4}(\mathbb{C}) \times \operatorname{GSp}_{4}(\mathbb{C})\right)^{\circ} \times \Gamma_{F}
$$

where, as before, $\Gamma_{F}$ is the absolute Galois group of $F$.

Our representation $\pi$ also decomposes as a tensor product $\pi=\pi_{1} \otimes \pi_{2}$, where $\pi_{1}$ and $\pi_{2}$ are irreducible admissible generic representations of $\operatorname{GSp}_{4}(F)$, the product of whose central characters is trivial. Since the Frobenius element is trivial here, the 
Satake parameter for $\pi$ will be a semisimple conjugacy class in ${ }^{L} G_{0}$ represented, say, by

$$
\left(x_{\pi_{1}}, y_{\pi_{2}}\right) \in\left(\operatorname{GSp}_{4}(\mathbb{C}) \times \operatorname{GSp}_{4}(\mathbb{C})\right)^{\circ}
$$

The representation $\rho_{\mathrm{TT}}$ is now the tensor product $\rho \otimes \rho$ as defined by (3.12), where $\rho$ is the standard representation of $\mathrm{Sp}_{4}(\mathbb{C})$, extended trivially on similitudes. So the local $L$-factor is just

$$
L\left(s, \pi_{1} \times \pi_{2}, \rho \otimes \rho\right)=\prod_{1 \leq i, j \leq 4}\left(1-x_{i} y_{j} q^{-s}\right)^{-1} .
$$

In [34], we find on p. 63 the identity

$$
\sum_{\lambda: \ell(\lambda) \leq n} s_{\lambda}\left(x_{1}, \ldots, x_{n}\right) s_{\lambda}\left(y_{1}, \ldots, y_{n}\right)=\prod_{i=1}^{n} \prod_{j=1}^{n}\left(1-x_{i} y_{j}\right)^{-1} .
$$

Take $n=4$ and recall that $s_{\lambda}$ is homogeneous of degree $|\lambda|\left(\right.$ so $s_{\lambda}\left(q^{-s} y_{1}, \ldots, q^{-s} y_{4}\right)=$ $q^{-s|\lambda|} s_{\lambda}\left(y_{1}, \ldots, y_{4}\right)$, for example). Let $x_{\pi_{1}}=\left(x_{1}, \ldots, x_{4}\right)$ and $y_{\pi_{2}}=\left(y_{1}, \ldots, y_{4}\right)$ be the Satake parameters for $\pi_{1}$ and $\pi_{2}$, respectively. Then writing (3.38) in terms of characters yields

$$
L\left(s, \pi_{1} \times \pi_{2}, \rho \otimes \rho\right)=\sum_{\lambda: \ell(\lambda) \leq 4} \chi_{\mathrm{GL}(4)}(\lambda)\left(x_{\pi_{1}}\right) \chi_{\mathrm{GL}(4)}(\lambda)\left(y_{\pi_{2}}\right) q^{-s|\lambda|} .
$$

So we may again apply our branching rule (3.21) to relate the $L$-factor to characters of $\mathrm{Sp}_{4}(\mathbb{C})$, and via a combinatorial fact to the integral above. The relevant identity here is the following. 
Proposition 3.2.3. Given a nonnegative integer $N$ and partitions $\mu=\left(\mu_{1}, \mu_{2}\right)$ and $\nu=\left(\nu_{1}, \nu_{2}\right)$ of length at most two, set $\delta_{\mu}=\mu_{1}-\mu_{2}, \delta_{\nu}=\nu_{1}-\nu_{2}$ and

$$
\begin{gathered}
M_{1}=\max \{|\mu|,|\nu|\}, \quad M_{2}=\min \{|\mu|,|\nu|\}, \\
m_{1}=\max \left\{\delta_{\nu}, \delta_{\mu}\right\}, \quad m_{2}=\min \left\{\delta_{\mu}, \delta_{\nu}\right\},
\end{gathered}
$$

and define

$$
D_{\mu, \nu, N}=\sum_{\substack{\lambda \vdash N \\
\ell(\lambda) \leq 4}} \sum_{\begin{array}{c}
\beta^{\prime} \text { even } \\
\alpha^{\prime} \text { even }
\end{array}}\left(c_{\mu, \beta}^{\lambda}-c_{\mu^{+}, \beta}^{\lambda}\right)\left(c_{\nu, \alpha}^{\lambda}-c_{\nu^{+}, \alpha}^{\lambda}\right) .
$$

Then we have

$$
\sum_{N=0}^{\infty} D_{\mu, \nu, 2 N+M_{1}} X^{N}=\frac{X^{\frac{1}{2}\left(m_{1}-m_{2}\right)}\left(1-X^{1+m_{2}}\right)\left(1-X^{1+\frac{1}{2}\left(M_{2}-m_{1}\right)}\right)}{(1-X)^{3}\left(1-X^{2}\right)}
$$

as functions in $X$.

Remark 3.2.4. (i). It is easy to see from the definition that $D_{\mu, \nu, N} \neq 0$ implies $N-|\mu|$ and $N-|\nu|$ are both even and $N \geq|\mu|,|\nu|$. Hence $D_{\mu, \nu, 2 N+M_{1}}$ is zero unless $M_{1} \equiv M_{2}(\bmod 2)$. Since $|\mu| \equiv \delta_{\mu}(\bmod 2)$ and the analogous statement holds for $\nu$, we actually have $D_{\mu, \nu, 2 N+M_{1}}=0$ unless $M_{1} \equiv M_{2} \equiv m_{1} \equiv m_{2}$ (mod 2). So the statement (3.41) is well-defined, that is, the exponents on the right-hand side are all integral.

(ii). We will show later that $D_{\mu, \nu, 2 N+M_{1}}=0$ for $M_{2}<m_{1}$ (see (3.51)) and that $D_{\mu, \nu, 2 N+M_{1}}=0$ for $N<\frac{1}{2}\left(m_{1}-m_{2}\right)$ (see (3.52)).

(iii). The right-hand side of (3.41) is exactly $P(\mu, \nu ; X)$ as in (3.35).

The proof will be given in Section 3.3. Now we may state the main result. 
Theorem 3.2.5. Let $s^{\prime}=(s+1) / 2$. Then

$$
\Psi^{*}\left(s, W_{1}^{\circ}, W_{2}^{\circ}, f_{s}^{\circ}\right)=L\left(s^{\prime}, \pi_{1} \times \pi_{2}, \rho \otimes \rho\right) .
$$

Proof. By (3.41), (3.35), Remark 3.2.4(i) and definition of $D_{\mu, \nu, 2 N+M_{1}}$,

$$
\begin{aligned}
P\left(\mu, \nu ; X^{2}\right) & =\sum_{N=0}^{\infty} D_{\mu, \nu, 2 N+M_{1}} X^{2 N} \\
& =\sum_{N=0}^{\infty} D_{\mu, \nu, N+M_{1}} X^{N}=\sum_{N=M_{1}}^{\infty} D_{\mu, \nu, N} X^{N-M_{1}} \\
& =\sum_{N=M_{1}}^{\infty} X^{N-M_{1}} \sum_{\substack{\lambda \wedge N \\
\ell(\lambda) \leq 4}} \sum_{\substack{\beta^{\prime} \text { even } \\
\alpha^{\prime} \text { even }}}\left(c_{\mu, \beta}^{\lambda}-c_{\mu^{+}, \beta}^{\lambda}\right)\left(c_{\nu, \alpha}^{\lambda}-c_{\nu^{+}, \alpha}^{\lambda}\right) \\
& =\sum_{\substack{\lambda \supseteq \mu, \nu \\
\ell(\lambda) \leq 4}} X^{|\lambda|-M_{1}} \sum_{\substack{\beta^{\prime} \text { even } \\
\alpha^{\prime} \text { even }}}\left(c_{\mu, \beta}^{\lambda}-c_{\mu^{+}, \beta}^{\lambda}\right)\left(c_{\nu, \alpha}^{\lambda}-c_{\nu^{+}, \alpha}^{\lambda}\right),
\end{aligned}
$$

so that by (3.34),

$$
\begin{aligned}
\Psi^{*}\left(s, W_{1}^{\circ}, W_{2}^{\circ}, f_{s}^{\circ}\right)=\sum_{\substack{\mu, \nu: M_{2} \geq m_{1} \\
\ell(\mu), \ell(\nu) \leq 2}} \chi_{\operatorname{Sp}(4)}(\mu)\left(x_{\pi_{1}}\right) \chi_{\operatorname{Sp}(4)}(\nu)\left(y_{\pi_{2}}\right) X^{M_{1}} P\left(\mu, \nu ; X^{2}\right) \\
=\sum_{\substack{\mu, \nu \\
\ell(\mu), \ell(\nu) \leq 2}} \chi_{\operatorname{Sp}(4)}(\mu)\left(x_{\pi_{1}}\right) \chi_{\operatorname{Sp}(4)}(\nu)\left(y_{\pi_{2}}\right) \\
\quad \cdot \sum_{\substack{\lambda \supseteq \mu, \nu \\
\ell(\lambda) \leq 4}} X^{|\lambda|} \sum_{\substack{\beta^{\prime} \text { even } \\
\alpha^{\prime} \text { even }}}\left(c_{\mu, \beta}^{\lambda}-c_{\mu^{+}, \beta}^{\lambda}\right)\left(c_{\nu, \alpha}^{\lambda}-c_{\nu^{+}, \alpha}^{\lambda}\right) \\
=\sum_{\lambda: \ell(\lambda) \leq 4} X^{|\lambda|}\left(\sum_{\mu: \ell(\mu) \leq 2} \chi_{\operatorname{Sp}(4)}(\mu)\left(x_{\pi_{1}}\right) \sum_{\beta^{\prime} \text { even }}\left(c_{\mu, \beta}^{\lambda}-c_{\mu^{+}, \beta}^{\lambda}\right)\right) \\
\quad \cdot\left(\sum_{\nu: \ell(\nu) \leq 2} \chi_{\operatorname{Sp}(4)}(\nu)\left(y_{\pi_{2}}\right) \sum_{\alpha^{\prime} \text { even }}\left(c_{\nu, \alpha}^{\lambda}-c_{\nu^{+}, \alpha}^{\lambda}\right)\right) \\
=\sum_{\lambda: \ell(\lambda) \leq 4} \chi_{\operatorname{GL}(4)}(\lambda)\left(x_{\pi_{1}}\right) \chi_{\mathrm{GL}(4)}(\lambda)\left(y_{\pi_{2}}\right) q^{-s^{\prime}|\lambda|}
\end{aligned}
$$




$$
=L\left(s^{\prime}, \pi_{1} \times \pi_{2}, \rho \otimes \rho\right)
$$

which is the desired result.

\subsection{Proof of combinatorial lemmas}

This section is devoted to proving Prop. 3.1.12, Prop. 3.1.13 and Prop. 3.2.3. Before giving the proofs, it will be convenient to cast the Littlewood-Richardson rule in a slightly different language.

Fix partitions $\lambda, \mu, \beta$ satisfying $\lambda \supseteq \mu, \lambda \supseteq \beta$ and $|\lambda|=|\mu|+|\beta|$. Call a tableau $T$ of skew-shape $\lambda / \mu$ and weight $\beta$ whose word is a lattice permutation, an $L R$-tableau. To such a tableau we may associate an integral matrix $A=\left(a_{i j}\right)=\left(a_{i j}(T)\right)$, where $a_{i j}$ is the number of symbols $j$ appearing in row $i$ of $T$. Because the word of $T$ is a lattice permutation, the symbol $j$ cannot appear until at least row $j$, starting from the top, so $A=A(T)$ is lower triangular. We can similarly translate all the conditions on an LR-tableau into conditions on such matrices:

Lemma 3.3.1. For $\lambda, \mu, \beta$ as above, set $n=\ell(\lambda)$. Then $c_{\beta, \mu}^{\lambda}=c_{\mu, \beta}^{\lambda}$ is the number of $n \times n$ integral matrices $A=\left(a_{i, j}\right)$ with nonnegative entries which are lower triangular and satisfy the following inequalities:

(i). $\sum_{j} a_{i, j}=\lambda_{i}-\beta_{i}$ and $\sum_{i} a_{i, j}=\mu_{j}$;

(ii). $\sum_{i=1}^{k} a_{i, j} \leq \sum_{i=1}^{k-1} a_{i, j-1}$ for $k \geq 1, j \geq 2$ (lattice permutation);

(iii). $\beta_{i}+\sum_{j=1}^{k} a_{i, j} \leq \beta_{i-1}+\sum_{j=1}^{k-1} a_{i-1, j}$ for $k \geq 1, i \geq 2$ (column-strictness). 
Proof of Prop. 3.1.12. Let $A=\left(a_{i j}\right)$ be a matrix as in the above lemma. Note that $\beta^{\prime}$ is even, so $\beta_{1}=\beta_{2}$ and $\beta_{3}=\beta_{4}$. The column-strict condition with $k=1$ and $i=2,4$ respectively shows that $a_{21}=0=a_{41}$. So our matrix has the following form:

$$
\begin{aligned}
& \begin{array}{l}
\lambda_{1}-\beta_{2} \\
\lambda_{2}-\beta_{2} \\
\lambda_{3}-\beta_{4} \\
\lambda_{4}-\beta_{4}
\end{array}\left(\begin{array}{ccccc}
a_{11} & & & \\
a_{31} & a_{32} & a_{33} & \\
0 & a_{42} & a_{43} & a_{44}
\end{array}\right) . \\
& \mu_{1} \quad \mu_{2} \quad \mu_{3} \quad \mu_{4}
\end{aligned}
$$

Then we get

$$
\begin{aligned}
& a_{11}=\lambda_{1}-\beta_{2}, \\
& a_{31}=\mu_{1}-a_{11}=\mu_{1}-\lambda_{1}+\beta_{2}=L_{1}, \\
& a_{22}=\lambda_{2}-\beta_{2}, \\
& a_{44}=\mu_{4} .
\end{aligned}
$$

The remaining four entries satisfy

$$
\begin{aligned}
& a_{32}+a_{33}=\lambda_{3}-\beta_{4}-a_{31} \\
& a_{42}+a_{43}=\lambda_{4}-\beta_{4}-\mu_{4}=-L_{4} \\
& a_{32}+a_{42}=\mu_{2}-\lambda_{2}+\beta_{2}=L_{2} \\
& a_{33}+a_{43}=\mu_{3},
\end{aligned}
$$

and one can check that the last equation is a consequence of the first three. 
Now set $a=a_{42} \geq 0$. Then

$$
\begin{aligned}
& a_{43}=-L_{4}-a \geq 0 \\
& a_{32}=L_{2}-a \geq 0 \\
& a_{33}=\mu_{3}-a_{43}=\mu_{3}+L_{4}+a \geq 0 .
\end{aligned}
$$

Now we write down all the inequalitites forced on the $a_{i j}$ in terms of $a$ :

$$
\left.\begin{array}{r}
L_{1}+\mu_{2}-\mu_{1}=L_{2}+\lambda_{2}-\lambda_{1}-\lambda_{3}=-L_{4}+\mu_{4}-\mu_{3} \\
L_{1}+L_{2}+\beta_{4}-\beta_{2} \\
0
\end{array}\right\} \leq a \leq\left\{\begin{array}{c}
L_{1} \\
L_{2} \\
-L_{3} \\
-L_{4} \\
L_{1}+L_{2}+\lambda_{2}-\lambda_{3}+\beta_{4}-\beta_{2} \\
=L_{1}+L_{3}+\mu_{2}-\mu_{3} \\
\mu_{2}-\mu_{3} .
\end{array}\right.
$$

So if any $\lambda_{i}=\lambda_{j}$ or $\mu_{i}=\mu_{j}$ for $i \neq j$, then $a$ and hence all the $a_{i j}$ will be completely determined, so $c_{\mu, \beta}^{\lambda} \leq 1$. This proves part (i).

Now suppose $\mu_{3}=\mu_{4}$. Then (3.42) gives $a=-L_{4}$, so applying the previous inequalities, eliminating redundances and using that $\sum_{i} L_{i}=0$ gives what we want. ${ }^{1}$

Before giving the next proof, it will be a helpful reference to rephrase Prop. 3.1.12 for the particular cases we need.

${ }^{1}$ Specifically, the redundant inequalities are $a=-L_{4} \leq \mu_{2}-\mu_{3}, a=-L_{4} \leq-L_{3},-L_{4}+\mu_{4}-\mu_{3} \leq$ $a \leq-L_{4}$. 
Corollary 3.3.2. Assume all the hypotheses of Prop. 3.1.12.

(i). If $\mu_{3}=\mu_{4}=0$, then $c_{\mu, \beta}^{\lambda}=1$ if and only if the following hold:

(a). $\lambda_{2}-\mu_{2}+\lambda_{4} \leq \beta_{2}+\beta_{4}$;

(b). $\lambda_{1}-\mu_{1}+\lambda_{4} \leq \beta_{2}+\beta_{4} \leq \lambda_{1}-\mu_{2}+\lambda_{4}$;

(c). $\lambda_{3} \leq \beta_{2}$.

(ii). If $\mu_{3}=\mu_{4}=1$, then $c_{\mu, \beta}^{\lambda}=1$ if and only if the following hold:

(a). $\lambda_{2}-\mu_{2}+\lambda_{4}-1 \leq \beta_{2}+\beta_{4}$;

(b). $\lambda_{1}-\mu_{1}+\lambda_{4}-1 \leq \beta_{2}+\beta_{4} \leq \lambda_{1}-\mu_{2}+\lambda_{4}-1$;

(c). $\lambda_{3} \leq \beta_{2}+1$;

(d). $\lambda_{2}-\beta_{2} \geq 1$ and $\lambda_{4}-\beta_{4} \geq 1$.

Remark 3.3.3. Consider the inequalities (a) and (b) above, under the same hypotheses but $\mu_{3}=\mu_{4}$ arbitrary. Since

$$
\beta_{2}+\beta_{4}=\beta_{1}+\beta_{3}=\frac{1}{2}|\beta|=\frac{1}{2}(|\lambda|-|\mu|),
$$

the statements are equivalent, respectively, to

$$
\begin{gathered}
\lambda_{2}+\lambda_{4}+\delta \leq \lambda_{1}+\lambda_{3}, \\
\lambda_{1}+\lambda_{4} \leq \lambda_{2}+\lambda_{3}+\delta \leq \lambda_{1}+\lambda_{4}+2 \delta,
\end{gathered}
$$

independent of the value of $\mu_{3}=\mu_{4}$. 


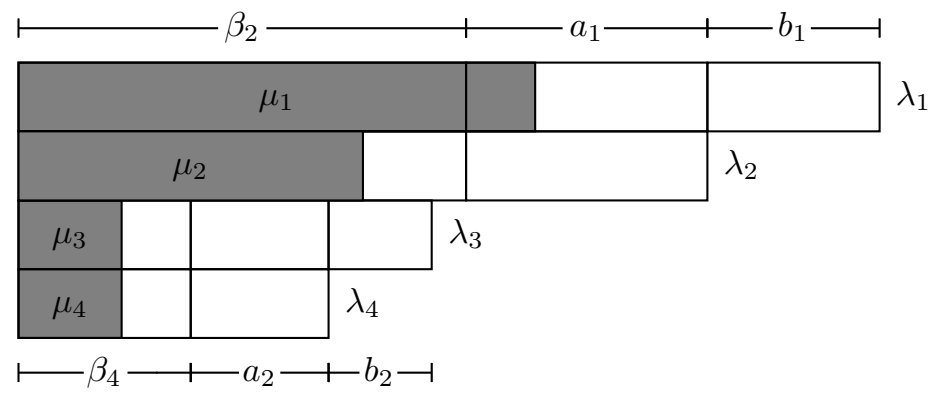

Figure 3.4: The partitions $\lambda, \beta$ and $\mu$

Recall that in order to calculate both $B_{\mu,|\mu|+2 N}$ and $D_{\mu, \nu, 2 N+M_{1}}$ we need to know sums of the form $\sum\left(c_{\mu, \beta}^{\lambda}-c_{\mu^{+}, \beta}^{\lambda}\right)$, where the sum is over $\beta^{\prime}$ even and $\mu^{+}=\mu+(0,0,1,1)$. We know that the coefficient $c_{\mu, \beta}^{\lambda}$ is zero unless $\lambda \supseteq \mu, \beta$ and $|\lambda|=|\mu|+|\beta|$. Suppose the triple $(\lambda, \mu, \beta)$ satisfies the hypotheses of Prop. 3.1.12. Then we may think of $\lambda$ as being built up from $\beta$ by adding $a_{1}$ blocks to each of rows 1 and $2, a_{2}$ blocks to each of rows 3 and $4, b_{1}$ blocks to row 1 and $b_{2}$ blocks to row 3 (see Figure 3.4). Then $\left(\right.$ recall $\beta_{1}=\beta_{2}$ and $\left.\beta_{3}=\beta_{4}\right)$

$$
\begin{aligned}
& \lambda_{1}=\beta_{2}+a_{1}+b_{1} \\
& \lambda_{2}=\beta_{2}+a_{1} \\
& \lambda_{3}=\beta_{4}+a_{2}+b_{2} \\
& \lambda_{4}=\beta_{4}+a_{2} .
\end{aligned}
$$

So we may translate the conditions of Prop. 3.1.12(ii) on $\lambda, \mu$ and $\beta$ into conditions 
on $a_{1}, a_{2}, b_{1}, b_{2}$. Fix $\lambda$ and $\mu$ and let $N=\beta_{2}+\beta_{4}=\frac{1}{2}(|\lambda|-|\mu|)$ and $r=\beta_{4}$, so $\beta_{2}=N-r$. Then the map

$$
(\lambda, \mu, \beta) \mapsto\left(a_{1}, a_{2}, b_{1}, b_{2}, r ; N\right)
$$

sets up a bijection between triples $(\lambda, \mu, \beta)$ such that $c_{\mu, \beta}^{\lambda} \neq 0$ and tuples $\left(a_{1}, a_{2}, b_{1}, b_{2}, r ; N\right)$ satisfying the corresponding conditions

$$
\begin{array}{cr}
2 a_{1}+b_{1}+2 a_{2}+b_{2}=|\mu|=2 \gamma+\delta & (|\lambda|-|\beta|=|\mu|) \\
\left|b_{1}-b_{2}\right| \leq \delta \leq b_{1}+b_{2} & \\
b_{1}+b_{2} \equiv \delta \quad(\bmod 2) & \\
a_{1}+a_{2} \leq \gamma & \\
a_{2}+b_{2} \leq N-2 r & (\text { by Remark 3.3.3) } \\
a_{1}+N-r \geq \max \left\{\mu_{1}-b_{1}, \gamma\right\} & \\
a_{1}, a_{2}, b_{1}, b_{2} \geq 0 & \\
0 \leq r \leq \llbracket \frac{N}{2} \rrbracket, &
\end{array}
$$

where we have set $\gamma=\mu_{2}$ and $\delta=\mu_{1}-\mu_{2}$, and $\llbracket \frac{N}{2} \rrbracket$ denotes the greatest integer function of $N / 2$. Note that the third and fourth equations are consequences of the first two. Let $\mathcal{C}(\lambda, \mu ; N)$ be the set of all tuples $\left(a_{1}, a_{2}, b_{1}, b_{2}, r ; N\right)$ satisfying the above conditions (so by Prop. 3.1.12, $c_{\mu, \beta}^{\lambda}=1$ ), so that

$$
\sharp \mathcal{C}(\lambda, \mu ; N)=\sum_{\beta^{\prime} \text { even }} c_{\mu, \beta}^{\lambda}
$$


Now consider the partially-defined map

$$
\left\{\begin{array}{l}
\mathcal{C}(\lambda, \mu ; N) \rightarrow \mathcal{C}\left(\lambda, \mu^{+} ; N-1\right) \\
\left(a_{1}, a_{2}, b_{1}, b_{2}, r ; N\right) \mapsto\left(a_{1}+1, a_{2}, b_{1}, b_{2}, r ; N-1\right) .
\end{array}\right.
$$

The equations satisfied by a tuple $\left(a_{1}^{\prime}, a_{2}^{\prime}, b_{1}^{\prime}, b_{2}^{\prime}, r^{\prime} ; N^{\prime}\right) \in \mathcal{C}\left(\lambda, \mu^{+} ; N-1\right)$ are

$$
\begin{array}{cr}
2 a_{1}^{\prime}+b_{1}^{\prime}+2 a_{2}^{\prime}+b_{2}^{\prime}=\left|\mu^{+}\right|=2 \gamma+2+\delta & \left(|\lambda|-|\beta|=\left|\mu^{+}\right|\right) \\
\left|b_{1}^{\prime}-b_{2}^{\prime}\right| \leq \delta \leq b_{1}^{\prime}+b_{2}^{\prime} & \\
b_{1}^{\prime}+b_{2}^{\prime} \equiv \delta \quad(\bmod 2) & (\text { by Remark 3.3.3) } \\
a_{1}^{\prime}+a_{2}^{\prime} \leq \gamma+1 & \text { (by Cor. 3.3.2(ii)(c)) } \quad\left(\lambda \supseteq \mu^{+}\right) \\
a_{2}^{\prime}+b_{2}^{\prime} \leq N-2 r^{\prime} & \\
a_{1}^{\prime}+N-1-r^{\prime} \geq \max \left\{\mu_{1}-b_{1}^{\prime}, \gamma\right\} & \\
b_{1}^{\prime}, b_{2}^{\prime} \geq 0 & \\
a_{1}^{\prime}, a_{2}^{\prime} \geq 1 & \text { (by Cor. 3.3.2(ii)(d)) } \\
0 \leq r^{\prime} \leq \llbracket \frac{N-1}{2} \rrbracket . &
\end{array}
$$

Comparing these with the equations for $\mathcal{C}(\lambda, \mu ; N)$ shows that the map (3.45) is defined on tuples $\mathbf{A}=\left(a_{1}, a_{2}, b_{1}, b_{2}, r ; N\right)$ with $a_{2} \geq 1$ and $r \leq \llbracket \frac{N-1}{2} \rrbracket$. Moreover, it 
is easy to see that this map is surjective where it is defined. Thus we have

$$
\begin{gathered}
\sum_{\beta^{\prime} \text { even }}\left(c_{\mu, \beta}^{\lambda}-c_{\mu^{+}, \beta}^{\lambda}\right)=\sharp \mathcal{C}(\lambda, \mu ; N)-\sharp \mathcal{C}\left(\lambda, \mu^{+} ; N-1\right) \\
=\sum_{\substack{\mathbf{A} \in \mathcal{C}(\lambda, \mu ; N) \\
a_{2}=0, r \leq \llbracket \frac{N-1}{2} \rrbracket}} 1+\sum_{\substack{\mathbf{A} \in \mathcal{C}(\lambda, \mu ; N) \\
N \text { even, } r=N / 2}} 1 \\
=\sum_{\substack{\mathbf{A} \in \mathcal{C}(\lambda, \mu ; N) \\
a_{2}=0, r \leq \llbracket \frac{N}{2} \rrbracket}} 1,
\end{gathered}
$$

where the last equality follows from the fact that for $\mathbf{A} \in \mathcal{C}(\lambda, \mu ; N), N$ even and $r=N / 2$ implies $a_{2}=0$. But there is at most one $\mathbf{A} \in \mathcal{C}(\lambda, \mu ; N)$ with $a_{2}=0$ : the equations for $\mathcal{C}(\lambda, \mu ; N)$ give

$$
\begin{gathered}
a_{2}=0 \\
b_{1}=\lambda_{1}-\lambda_{2} \\
b_{2}=\lambda_{3}-\lambda_{4} \\
r=\lambda_{4} \\
a_{1}=\lambda_{2}+\lambda_{4}-N .
\end{gathered}
$$

Hence we have shown the

Lemma 3.3.4. Let $\lambda, \mu$ be partitions such that $\lambda \supseteq \mu, \ell(\lambda) \leq 4$ and $\ell(\mu) \leq 2$. Then

$$
\sum_{\beta^{\prime} \text { even }}\left(c_{\mu, \beta}^{\lambda}-c_{\mu^{+}, \beta}^{\lambda}\right)
$$

is either 0 or 1 , and is 1 if and only if there is an $\mathbf{A} \in \mathcal{C}(\lambda, \mu ; N)$ such that $a_{2}=0$. 
Corollary 3.3.5. Let $\mu$ have length at most two. Then if $\nu=\mu$,

$$
D_{\mu, \nu, 2 N+M_{1}}=B_{\mu,|\mu|+2 N} .
$$

Proof. For $\nu=\mu, M_{1}=|\mu|$ and so

$$
\begin{aligned}
D_{\mu, \nu, 2 N+M_{1}} & =D_{\mu, \mu, 2 N+|\mu|}=\sum_{\substack{\lambda \vdash|\mu|+2 N \\
\ell(\lambda) \leq 4}}\left(\sum_{\beta^{\prime} \text { even }}\left(c_{\mu, \beta}^{\lambda}-c_{\mu^{+}, \beta}^{\lambda}\right)\right)^{2} \\
& =\sum_{\substack{\lambda \vdash|\mu|+2 N \\
\ell(\lambda) \leq 4}} \sum_{\beta^{\prime} \text { even }}\left(c_{\mu, \beta}^{\lambda}-c_{\mu^{+}, \beta}^{\lambda}\right)=B_{\mu,|\mu|+2 N .} .
\end{aligned}
$$

Notice also that in the case $\mu=\nu$, the right-hand side of (3.41) simplifies to the right-hand side of (3.23), so that Prop. 3.1.13 follows from Prop. 3.2.3.

Proof of Prop. 3.2.3. The plan of proof is as follows. First, we compute the $N$-th coefficient of the right-hand side of (3.41). Second, by Lemma 3.3.4 determining $D_{\mu, \nu, 2 N+M_{1}}$ essentially reduces to counting the set of tuples $\mathbf{A}=\left(a_{1}, a_{2}, b_{1}, b_{2}, r ; N\right) \in$ $\mathcal{C}(\lambda, \mu ; N)$ with $a_{2}=0$. We show the resulting sum simplifies to the aforementioned $N$-th coefficient.

For the remainder of the proof, set $\varepsilon^{\prime}=\frac{1}{2}\left(m_{1}-m_{2}\right), \gamma=\frac{1}{2}\left(M_{2}-m_{2}\right), \delta=\frac{1}{2}\left(m_{1}+m_{2}\right)$ and $\varepsilon=\frac{1}{2}\left(M_{2}+m_{2}\right)$, so $\gamma+\delta=\varepsilon+\varepsilon^{\prime}$. (Note that if $\mu=\nu$, this agrees with the previous definitions of $\gamma$ and $\delta$, and $\varepsilon^{\prime}=0$.) Also set $N_{\mu}=N+\frac{1}{2}\left(M_{1}-|\mu|\right)$ and $N_{\nu}=N+\frac{1}{2}\left(M_{1}-|\nu|\right)$, so that

$$
2 N_{\mu}+|\mu|=2 N+M_{1}=2 N_{\nu}+|\nu| .
$$


For a statement $P$, we will use the notation

$$
[P]= \begin{cases}1 & \text { if } P \text { is true } \\ 0 & \text { if } P \text { is false }\end{cases}
$$

As above, $\llbracket n \rrbracket$ denotes the greatest integer function of $n$. Finally, in accordance with standard convention, empty sums are interpreted as zero.

Now the right-hand side of (3.41) is

$$
\frac{X^{\varepsilon^{\prime}}\left(1-X^{1+\gamma-\varepsilon^{\prime}}\right)\left(1-X^{1+\delta-\varepsilon^{\prime}}\right)}{(1-X)^{3}\left(1-X^{2}\right)}=\frac{P(X)}{(1-X)\left(1-X^{2}\right)},
$$

where

$$
P(X)=X^{\varepsilon^{\prime}}\left(1+X+\cdots+X^{\gamma-\varepsilon^{\prime}}\right)\left(1+X+\cdots+X^{\delta-\varepsilon^{\prime}}\right)=\sum_{k=0}^{\mu_{1}} d_{k} X^{k}
$$

and with $k_{0}=\min \{\gamma, \delta\}$,

$$
d_{k}= \begin{cases}1+k-\varepsilon^{\prime} & \text { if } \varepsilon^{\prime} \leq k \leq k_{0} \\ 1+k_{0}-\varepsilon^{\prime} & \text { if } k_{0} \leq k \leq \varepsilon+\varepsilon^{\prime}-k_{0} \\ 1+\varepsilon-k & \text { if } \varepsilon+\varepsilon^{\prime}-k_{0} \leq k \leq \varepsilon \\ 0 & \text { otherwise. }\end{cases}
$$

The rational function $\left((1-X)\left(1-X^{2}\right)\right)^{-1}$ is the generating function for the numbers $p_{2}(n)$, the number of partitions of $n$ with length at most 2 . So the $N$-th coefficient of (3.47) is

$$
\sum_{k=0}^{N} p_{2}(N-k) d_{k}=\sum_{k=\varepsilon^{\prime}}^{\min \{N, \varepsilon\}} p_{2}(N-k) d_{k}
$$




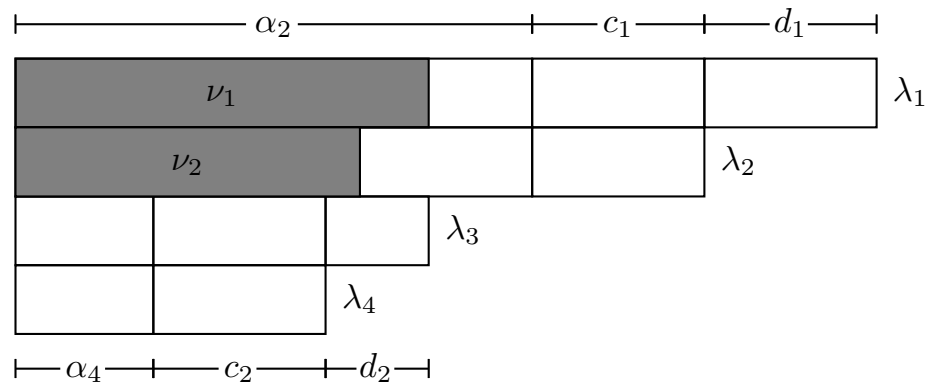

Figure 3.5: The partitions $\lambda, \alpha$ and $\nu$

Now by Lemma 3.3.4, $D_{\mu, \nu, 2 N+M_{1}}$ counts pairs of triples $\{(\lambda, \mu, \beta),(\lambda, \nu, \alpha)\}$ (all with the same $\mu$ and $\nu)$ for which $(\lambda, \mu, \beta)$ and $(\lambda, \nu, \alpha)$ simultaneously have realizations as tuples $\mathbf{A}=\left(a_{1}, a_{2}, b_{1}, b_{2}, r ; N_{\mu}\right) \in \mathcal{C}\left(\lambda, \mu ; N_{\mu}\right)$ with $a_{2}=0$ and $\mathbf{C}=$ $\left(c_{1}, c_{2}, d_{1}, d_{2}, t ; N_{\nu}\right) \in \mathcal{C}\left(\lambda, \nu ; N_{\nu}\right)$ with $c_{2}=0$, respectively. (See Figures 3.4 and 3.5.) This gives

$$
\begin{aligned}
& \lambda_{1}=\beta_{2}+a_{1}+b_{1}=\alpha_{2}+c_{1}+d_{1} \\
& \lambda_{2}=\beta_{2}+a_{1}=\alpha_{2}+c_{1} \\
& \lambda_{3}=\beta_{4}+a_{2}+b_{2}=\alpha_{4}+c_{2}+d_{2} \\
& \lambda_{4}=\beta_{4}+a_{2}=\alpha_{4}+c_{2} .
\end{aligned}
$$

So immediately we see that if $a_{2}=c_{2}=0$,

$$
\begin{aligned}
& b_{1}=d_{1}, b_{2}=d_{2} \\
& \beta_{4}=r=\alpha_{4}=t
\end{aligned}
$$


and since $\beta_{2}+\beta_{4}=N_{\mu}$ and $\alpha_{2}+\alpha_{4}=N_{\nu}$,

$$
a_{1}+N_{\mu}-r=\lambda_{2}=c_{1}+N_{\nu}-t \quad \Longrightarrow \quad a_{1}+N_{\mu}=c_{1}+N_{\nu} .
$$

So our integers $a_{1}, b_{1}, b_{2}, c_{1}, r$ must satisfy the following conditions:

$$
\begin{gathered}
2 a_{1}+b_{1}+b_{2}=|\mu| \\
2 c_{1}+b_{1}+b_{2}=|\nu| \\
\left|b_{1}-b_{2}\right| \leq \delta_{\mu}, \delta_{\nu} \leq b_{1}+b_{2} \\
b_{1}+b_{2} \equiv \delta_{\mu} \equiv \delta_{\nu} \quad(\bmod 2) \\
a_{1} \leq \mu_{2} \\
c_{1} \leq \nu_{2} \\
b_{2} \leq \min \left\{N_{\mu}-2 r, N_{\nu}-2 r\right\} \\
a_{1}+N_{\mu}-r \geq \max \left\{\mu_{1}-b_{1}, \mu_{2}\right\} \\
c_{1}+N_{\nu}-r \geq \max \left\{\nu_{1}-b_{1}, \nu_{2}\right\} \\
a_{1}, b_{1}, b_{2}, c_{1} \geq 0 \\
0 \leq r \leq \llbracket \frac{N}{2} \rrbracket .
\end{gathered}
$$

Set $a=a_{1}+N_{\mu}-N=c_{1}+N_{\nu}-N$. Then writing these conditions in terms of $a, b_{1}, b_{2}$ 
gives

$$
\begin{gathered}
2 a+b_{1}+b_{2}=M_{1} \\
\left|b_{1}-b_{2}\right| \leq m_{2} \leq m_{1} \leq b_{1}+b_{2} \\
b_{1}+b_{2} \equiv m_{1} \equiv m_{2} \quad(\bmod 2) \\
a \leq \frac{1}{2}\left(M_{1}-m_{1}\right) \\
b_{2} \leq N-2 r \\
a+N-r \geq \max \left\{\mu_{1}-b_{1}, \nu_{1}-b_{1}, \mu_{2}, \nu_{2}\right\} \\
a \geq \frac{1}{2}\left(M_{1}-M_{2}\right) \\
b_{1}, b_{2} \geq 0 \\
0 \leq r \leq \llbracket \frac{N}{2} \rrbracket .
\end{gathered}
$$

Since $b_{1}$ is determined by $a$ and $b_{2}$, we may count such tuples by summing over $r, a, b_{2}$ satisfying

- $0 \leq r \leq \llbracket \frac{N}{2} \rrbracket$

- $\max \left\{\frac{1}{2}\left(M_{1}-M_{2}\right), \mu_{2}+N-r, \nu_{2}+N-r\right\} \leq a \leq \frac{1}{2}\left(M_{1}-m_{1}\right)$

- $0 \leq b_{2} \leq N-2 r$.

One immediate consequence of the summation range for $a$ is that

$$
D_{\mu, \nu, 2 N+M_{1}}=0 \text { if } M_{2}<m_{1} .
$$

We must also ensure the constraints on $b_{1}=M_{1}-2 a-b_{2}$ are met: 
(i). $b_{2}-m_{2} \leq b_{1} \leq b_{2}+m_{2}$ if and only if

$$
\frac{1}{2}\left(M_{1}-m_{2}\right)-a \leq b_{2} \leq \frac{1}{2}\left(M_{1}+m_{2}\right)-a .
$$

Note that the leftmost term is nonnegative, since $a \leq \frac{1}{2}\left(M_{1}-m_{1}\right) \leq \frac{1}{2}\left(M_{1}-m_{2}\right)$.

(ii). $b_{1} \geq m_{1}-b_{2}$ if and only if $a \leq \frac{1}{2}\left(M_{1}-m_{1}\right)$, which already holds.

(iii). The previous constraints show $b_{1} \geq m_{1}-b_{2} \geq m_{2}-b_{2}$ and $b_{1} \geq b_{2}-m_{2}$, so already $b_{1} \geq 0$.

(iv). $b_{1} \geq \max \left\{\mu_{1}-N+r-a, \nu_{1}-N+r-a\right\}$ if and only if $b_{2} \leq \min \left\{N+M_{1}-\right.$ $\left.\mu_{1}-a-r, N+M_{1}-\nu_{1}-a-r\right\}=N+M_{1}-a-r-\max \left\{\mu_{1}, \nu_{1}\right\}$.

So the $b_{2}$-range should actually be $\frac{1}{2}\left(M_{1}-m_{2}\right)-a \leq b_{2} \leq \min \left\{N-2 r, \frac{1}{2}\left(M_{1}+m_{2}\right)-a, N+M_{1}-a-r-\max \left\{\mu_{1}, \nu_{1}\right\}\right\}$.

But some of these constraints are redundant:

(i). $N-2 r \leq N+M_{1}-a-r-\max \left\{\mu_{1}, \nu_{1}\right\}$ if and only if $a \leq M_{1}+r-\max \left\{\mu_{1}, \nu_{1}\right\}(*)$. Now we already have $a \leq \frac{1}{2}\left(M_{1}-m_{1}\right)$, and $\frac{1}{2}\left(M_{1}-m_{1}\right) \leq M_{1}+r-\max \left\{\mu_{1}, \nu_{1}\right\}$ if and only if $\frac{1}{2}\left(M_{1}+m_{1}\right)-\max \left\{\mu_{1}, \nu_{1}\right\}+r \geq 0$. But clearly

$$
M_{1}+m_{1} \geq \max \left\{|\mu|+\delta_{\mu},|\nu|+\delta_{\nu}\right\} \geq \max \left\{2 \mu_{1}, 2 \nu_{1}\right\},
$$

and $r \geq 0$. So $(*)$ always holds and thus the first statement does as well.

(ii). The $b_{2}$-range is nonempty $\Longleftrightarrow$ either $N-2 r \geq \frac{1}{2}\left(M_{1}+m_{2}\right)-a$ or $N-2 r \geq$ $\frac{1}{2}\left(M_{1}-m_{2}\right)-a$. Since the second inequality is weaker in $a$, we see that the $b_{2}$-range is nonempty if and only if $a \geq \frac{1}{2}\left(M_{1}-m_{2}\right)-N+2 r$. Now

$$
M_{1}-m_{2} \geq \max \left\{|\mu|-\delta_{\mu},|\nu|-\delta_{\nu}\right\}=\max \left\{2 \mu_{2}, 2 \nu_{2}\right\},
$$


so $\frac{1}{2}\left(M_{1}-m_{2}\right)-N+2 r \geq \max \left\{\mu_{2}-N+r, \nu_{2}-N+r\right\}$. Thus the range of summation for $a$ is

$$
\max \left\{\frac{1}{2}\left(M_{1}-M_{2}\right), \frac{1}{2}\left(M_{1}-m_{2}\right)-N+2 r\right\} \leq a \leq \frac{1}{2}\left(M_{1}-m_{1}\right)
$$

(iii). Finally, the $a$-range is nonempty $\Longleftrightarrow$ either $\frac{1}{2}\left(M_{1}-m_{2}\right)-N+2 r \leq \frac{1}{2}\left(M_{1}-M_{2}\right)$ or $\frac{1}{2}\left(M_{1}-m_{2}\right)-N+2 r \leq \frac{1}{2}\left(M_{1}-m_{1}\right)$. Since the second inequality is weaker in $r$, we conclude that the $a$-range is nonempty if and only if $r \leq \llbracket \frac{N-\frac{1}{2}\left(m_{1}-m_{2}\right)}{2} \rrbracket=$ $\llbracket \frac{N-\varepsilon^{\prime}}{2} \rrbracket$. In particular,

$$
D_{\mu, \nu, 2 N+M_{1}}=0 \text { if } N<\varepsilon^{\prime}=\frac{1}{2}\left(m_{1}-m_{2}\right)
$$

Hence the summation is over $r, a, b=b_{2}$ such that

- $0 \leq r \leq \llbracket \frac{N-\varepsilon^{\prime}}{2} \rrbracket$

- $\max \left\{\frac{1}{2}\left(M_{1}-M_{2}\right), \frac{1}{2}\left(M_{1}-m_{2}\right)-N+2 r\right\} \leq a \leq \frac{1}{2}\left(M_{1}-m_{1}\right)$

- $\frac{1}{2}\left(M_{1}-m_{2}\right)-a \leq b \leq \min \left\{N-2 r, \frac{1}{2}\left(M_{1}+m_{2}\right)-a\right\}$.

Finally, for later reference we give the various cutoffs in summation:

b: $\frac{1}{2}\left(M_{1}+m_{2}\right)-a \leq N-2 r \Longleftrightarrow a \geq \frac{1}{2}\left(M_{1}+m_{2}\right)-N+2 r$.

a: (i). $\frac{1}{2}\left(M_{1}-m_{2}\right)-N+2 r \leq \frac{1}{2}\left(M_{1}-M_{2}\right)$ if and only if

$$
r \leq \llbracket \frac{N-\frac{1}{2}\left(M_{2}-m_{2}\right)}{2} \rrbracket=\llbracket \frac{N-\gamma}{2} \rrbracket .
$$

(ii). $\frac{1}{2}\left(M_{1}-M_{2}\right) \leq \frac{1}{2}\left(M_{1}+m_{2}\right)-N+2 r \leq \frac{1}{2}\left(M_{1}-m_{1}\right)$ if and only if $\frac{N-\frac{1}{2}\left(M_{2}+m_{2}\right)}{2} \leq r \leq \frac{N-\frac{1}{2}\left(m_{1}+m_{2}\right)}{2}$ if and only if

$$
\frac{N-\varepsilon}{2} \leq r \leq \llbracket \frac{N-\delta}{2} \rrbracket
$$




$$
r: \llbracket \frac{N-\delta}{2} \rrbracket \leq \llbracket \frac{N-\gamma}{2} \rrbracket \Longleftrightarrow \gamma \leq \delta .
$$

So we must consider the cases $\gamma \leq \delta$ and $\gamma \geq \delta$ separately.

Case $\gamma \leq \delta$.

$$
\begin{aligned}
& D_{\mu, \nu, 2 N+M_{1}}= \\
& {[N \geq \varepsilon] \sum_{r=0}^{\llbracket \frac{N-\varepsilon}{2} \rrbracket} \sum_{a=\frac{1}{2}\left(M_{1}-M_{2}\right)}^{\frac{1}{2}\left(M_{1}-m_{1}\right)}\left(1+\left(\frac{1}{2}\left(M_{1}+m_{2}\right)-a\right)-\left(\frac{1}{2}\left(M_{1}-m_{2}\right)-a\right)\right)}
\end{aligned}
$$

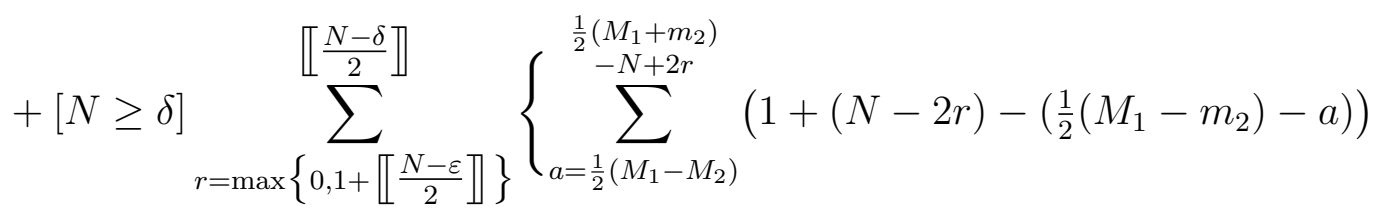

$$
\begin{aligned}
& \left.+\sum_{\substack{a=\frac{1}{2}\left(M_{1}+m_{2}\right) \\
-N+2 r+1}}^{\frac{1}{2}\left(M_{1}-m_{1}\right)}\left(1+\left(\frac{1}{2}\left(M_{1}+m_{2}\right)-a\right)-\left(\frac{1}{2}\left(M_{1}-m_{2}\right)-a\right)\right)\right\} \\
& +[N \geq \gamma] \sum_{r=\max }^{\llbracket \frac{N-\gamma}{2} \rrbracket} \sum_{\left.0,1+\llbracket \frac{N-\delta}{2} \rrbracket\right\}}^{\frac{1}{2}\left(M_{1}-m_{1}\right)}\left(1+(N-2 r)-\left(\frac{1}{2}\left(M_{1}-m_{2}\right)-a\right)\right) \\
& +\left[N \geq \varepsilon^{\prime}\right] \sum_{r=\max }^{\llbracket \frac{N-\varepsilon^{\prime}}{2} \rrbracket} \sum_{\left.0,1+\llbracket \frac{N-\delta}{2} \rrbracket\right\}}^{\substack{\frac{1}{2}\left(M_{1}-m_{1}\right) \\
a=\frac{1}{2}\left(M_{1}-m_{2}\right) \\
-N+2 r}}\left(1+(N-2 r)-\left(\frac{1}{2}\left(M_{1}-m_{2}\right)-a\right)\right)
\end{aligned}
$$




$$
\begin{aligned}
& =[N \geq \varepsilon] \sum_{r=0}^{\llbracket \frac{N-\varepsilon}{2} \rrbracket} \sum_{a=\frac{1}{2}\left(M_{1}-M_{2}\right)}^{\frac{1}{2}\left(M_{1}-m_{1}\right)}\left(1+m_{2}\right) \\
& +[N \geq \delta] \sum_{r=\max \left\{0,1+\llbracket \frac{N-\varepsilon}{2} \rrbracket\right\}}^{\llbracket \frac{N-\delta}{2} \rrbracket} \\
& \left\{\sum_{a=\frac{1}{2}\left(M_{1}-M_{2}\right)}^{\substack{\frac{1}{2}\left(M_{1}+m_{2}\right) \\
-N+2 r}}\left(1+a+N-2 r-\frac{1}{2}\left(M_{1}-m_{2}\right)\right)+\sum_{\substack{a=\frac{1}{2}\left(M_{1}+m_{2}\right) \\
-N+2 r+1}}^{\frac{1}{2}\left(M_{1}-m_{1}\right)}\left(1+m_{2}\right)\right\} \\
& +[N \geq \gamma] \sum_{r=\max }^{\llbracket \frac{N-\gamma}{2} \rrbracket} \sum_{\left.0,1+\llbracket \frac{N-\delta}{2} \rrbracket\right\}}^{\frac{1}{2}\left(M_{1}-m_{1}\right)}\left(1+a+N-2 r-\frac{1}{2}\left(M_{1}-m_{2}\right)\right) \\
& +\left[N \geq \varepsilon^{\prime}\right] \sum_{r=\max }^{\llbracket \frac{N-\varepsilon^{\prime}}{2} \rrbracket} \sum_{\left.0,1+\llbracket \frac{N-\delta}{2} \rrbracket\right\}}^{\substack{\frac{1}{2}\left(M_{1}-m_{1}\right) \\
a=\frac{1}{2}\left(M_{1}-m_{2}\right) \\
-N+2 r}}\left(1+a+N-2 r-\frac{1}{2}\left(M_{1}-m_{2}\right)\right)
\end{aligned}
$$




$$
\begin{aligned}
& =[N \geq \varepsilon] \sum_{r=0}^{\llbracket \frac{N-\varepsilon}{2} \rrbracket} \sum_{a=0}^{\frac{1}{2}\left(M_{2}-m_{1}\right)}\left(1+m_{2}\right) \\
& +[N \geq \delta] \sum_{r=\max }^{\llbracket \frac{N-\delta}{2} \rrbracket}\left\{\sum_{\substack{a=N-2 r \\
-\frac{1}{2}\left(M_{2}-m_{2}\right)}}^{m_{2}}(1+a)+\sum_{m_{2}+1}^{-\frac{1}{2}\left(m_{1}-m_{2}\right)}\left(1+m_{2}\right)\right\} \\
& +[N \geq \gamma] \sum_{r=\max \left\{0,1+\llbracket \frac{N-\delta}{2} \rrbracket\right\}}^{\llbracket \frac{N-\gamma}{2} \rrbracket} \sum_{a=N-2 r-\frac{1}{2}\left(M_{2}-m_{2}\right)}^{N-2 r-\frac{1}{2}\left(m_{1}-m_{2}\right)}(1+a) \\
& +\left[N \geq \varepsilon^{\prime}\right] \sum_{r=\max }^{\llbracket \frac{N-\varepsilon^{\prime}}{2} \rrbracket} \sum_{a=0}^{N-2 r-\frac{1}{2}\left(m_{1}-m_{2}\right)}(1+a) \\
& =[N \geq \varepsilon] \sum_{r=0}^{\llbracket \frac{N-\varepsilon}{2} \rrbracket} \sum_{a=0}^{\gamma-\varepsilon^{\prime}}\left(1+\delta-\varepsilon^{\prime}\right) \\
& +[N \geq \delta] \sum_{r=\max \left\{0,1+\llbracket \frac{N-\varepsilon}{2} \rrbracket\right\}}^{\llbracket \frac{N-\delta}{2} \rrbracket}\left\{\sum_{a=N-2 r-\gamma}^{\delta-\varepsilon^{\prime}}(1+a)+\sum_{\delta-\varepsilon^{\prime}+1}^{N-2 r-\varepsilon^{\prime}}\left(1+\delta-\varepsilon^{\prime}\right)\right\} \\
& +[N \geq \gamma] \sum_{r=\max }^{\left.\llbracket 0,1+\llbracket \frac{N-\delta}{2} \rrbracket\right\}^{a=N-2 r-\gamma}} \sum^{\frac{N-\gamma}{2} \rrbracket}(1+a) \\
& +\left[N \geq \varepsilon^{\prime}\right] \sum_{r=\max }^{\llbracket \frac{\frac{N-\varepsilon^{\prime}}{2} \rrbracket}{\left.20,1+\llbracket \frac{N-\delta}{2} \rrbracket\right\}}} \sum_{a=0}^{N-2 r-\varepsilon^{\prime}}(1+a) .
\end{aligned}
$$


Case $\delta \leq \gamma$

$$
\begin{aligned}
& D_{\mu, \nu, 2 N+M_{1}}= \\
& {[N \geq \varepsilon] \sum_{r=0}^{\llbracket \frac{N-\varepsilon}{2} \rrbracket} \sum_{a=\frac{1}{2}\left(M_{1}-M_{2}\right)}^{\frac{1}{2}\left(M_{1}-m_{1}\right)}\left(1+\left(\frac{1}{2}\left(M_{1}+m_{2}\right)-a\right)-\left(\frac{1}{2}\left(M_{1}-m_{2}\right)-a\right)\right)}
\end{aligned}
$$

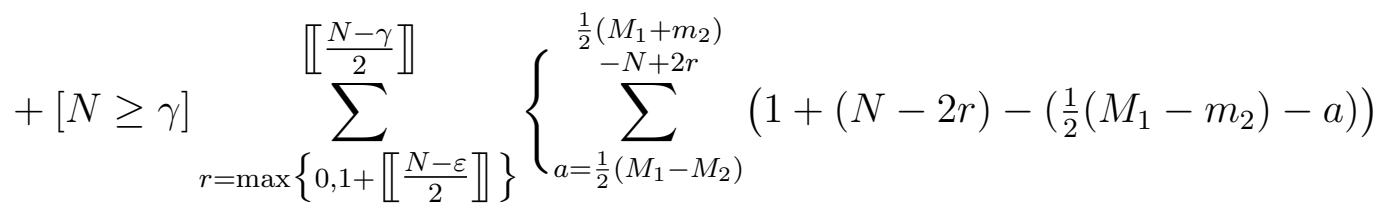

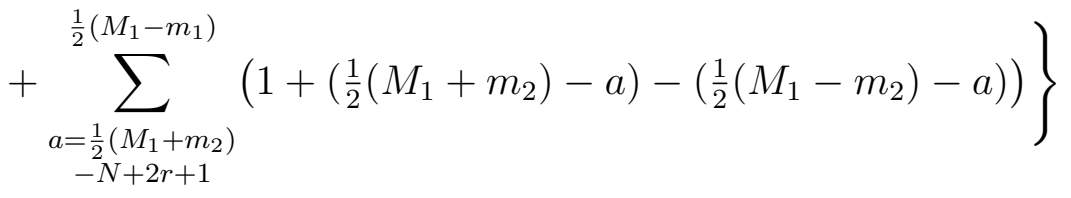

$$
\begin{aligned}
& +[N \geq \delta] \sum_{r=\max }^{\llbracket \frac{1-\delta-\delta}{2} \rrbracket}\left\{\sum_{\substack{\frac{N}{2}\left(M_{1}+m_{2}\right) \\
-N+2 r}}^{\substack{\frac{1}{2} \rrbracket \\
a=\frac{1}{2}\left(M_{1}-m_{2}\right) \\
-N+2 r}}\left(1+(N-2 r)-\left(\frac{1}{2}\left(M_{1}-m_{2}\right)-a\right)\right)\right. \\
& \left.+\sum_{\frac{1}{2}\left(M_{1}+m_{2}\right)}^{\frac{1}{2}\left(M_{1}-m_{1}\right)}\left(1+\left(\frac{1}{2}\left(M_{1}+m_{2}\right)-a\right)-\left(\frac{1}{2}\left(M_{1}-m_{2}\right)-a\right)\right)\right\} \\
& +\left[N \geq \varepsilon^{\prime}\right] \sum_{r=\max }^{\llbracket \frac{N-\varepsilon^{\prime}}{2} \rrbracket} \sum_{\left.0,1+\llbracket \frac{N-\delta}{2} \rrbracket\right\}}^{\substack{\frac{1}{2}\left(M_{1}-m_{1}\right) \\
a=\frac{1}{2}\left(M_{1}-m_{2}\right) \\
-N+2 r}}\left(1+(N-2 r)-\left(\frac{1}{2}\left(M_{1}-m_{2}\right)-a\right)\right)
\end{aligned}
$$




$$
\begin{aligned}
& =[N \geq \varepsilon] \sum_{r=0}^{\llbracket \frac{N-\varepsilon}{2} \rrbracket} \sum_{a=\frac{1}{2}\left(M_{1}-M_{2}\right)}^{\frac{1}{2}\left(M_{1}-m_{1}\right)}\left(1+m_{2}\right) \\
& +[N \geq \gamma] \sum_{r=\max }^{\llbracket \frac{N-\gamma}{2} \rrbracket} \sum_{\left.0,1+\llbracket \frac{N-\varepsilon}{2} \rrbracket\right\}}^{\llbracket} \\
& \left\{\sum_{a=\frac{1}{2}\left(M_{1}-M_{2}\right)}^{\substack{\frac{1}{2}\left(M_{1}+m_{2}\right) \\
-N+2 r}}\left(1+a+N-2 r-\frac{1}{2}\left(M_{1}-m_{2}\right)\right)+\sum_{\substack{a=\frac{1}{2}\left(M_{1}+m_{2}\right) \\
-N+2 r+1}}^{\frac{1}{2}\left(M_{1}-m_{1}\right)}\left(1+m_{2}\right)\right\} \\
& +[N \geq \delta] \sum_{r=\max \left\{0,1+\llbracket \frac{N-\gamma}{2} \rrbracket\right\}}^{\llbracket \frac{N-\delta}{2} \rrbracket} \\
& \left\{\sum_{\substack{a=\frac{1}{2}\left(M_{1}-m_{2}\right) \\
-N+2 r}}^{\substack{\frac{1}{2}\left(M_{1}+m_{2}\right) \\
-N+2 r}}\left(1+a+N-2 r-\frac{1}{2}\left(M_{1}-m_{2}\right)\right)+\sum_{\substack{\frac{1}{2}\left(M_{1}+m_{2}\right) \\
-N+2 r+1}}^{\frac{1}{2}\left(M_{1}-m_{1}\right)}\left(1+m_{2}\right)\right\} \\
& +\left[N \geq \varepsilon^{\prime}\right] \sum_{r=\max }^{\llbracket \frac{N-\varepsilon^{\prime}}{2} \rrbracket} \sum_{\left.0,1+\llbracket \frac{N-\delta}{2} \rrbracket\right\}}^{\frac{1}{2}\left(M_{1}-m_{1}\right)}\left(1+a+N-2 r-\frac{1}{2}\left(M_{1}-m_{2}\right)\right)
\end{aligned}
$$




$$
\begin{aligned}
& =[N \geq \varepsilon] \sum_{r=0}^{\llbracket \frac{N-\varepsilon}{2} \rrbracket} \sum_{a=0}^{\frac{1}{2}\left(M_{2}-m_{1}\right)}\left(1+m_{2}\right) \\
& +[N \geq \gamma] \sum_{r=\max }^{\llbracket \frac{N-\gamma}{2} \rrbracket}\left\{\sum_{\substack { a=N-2 r \\
\begin{subarray}{c}{a \\
-\frac{1}{2}\left(M_{2}-m_{2}\right){ a = N - 2 r \\
\begin{subarray} { c } { a \\
- \frac { 1 } { 2 } ( M _ { 2 } - m _ { 2 } ) } }\end{subarray}}^{m_{2}}(1+a)+\sum_{m_{2}+1}^{-\frac{N-\varepsilon}{2} \rrbracket}\left(1+m_{2}\right)\right\} \\
& +[N \geq \delta] \sum_{r=\max }^{\llbracket \frac{N-\delta}{2} \rrbracket}\left\{\sum_{a=0}^{m_{2}}(1+a)+\sum_{a=m_{2}+1}^{N-2 r-\frac{1}{2}\left(m_{1}-m_{2}\right)}\left(1+m_{2}\right)\right\} \\
& +\left[N \geq \varepsilon^{\prime}\right] \sum_{r=\max \left\{0,1+\llbracket \frac{N-\delta}{2} \rrbracket\right\}}^{\llbracket \frac{N-\varepsilon^{\prime}}{2} \rrbracket} \sum_{a=0}^{N-2 r-\frac{1}{2}\left(m_{1}-m_{2}\right)}(1+a) \\
& =[N \geq \varepsilon] \sum_{r=0}^{\llbracket \frac{N-\varepsilon}{2} \rrbracket} \sum_{a=0}^{\gamma-\varepsilon^{\prime}}\left(1+\delta-\varepsilon^{\prime}\right) \\
& +[N \geq \gamma] \sum_{r=\max }^{\llbracket \frac{N-\gamma}{2} \rrbracket}\left\{\sum_{\left.0,1+\llbracket \frac{N-\varepsilon}{2} \rrbracket\right\}}^{\delta-\varepsilon^{\prime}}(1+a)+\sum_{\delta=N-2 r-\gamma}^{N-2 r-\varepsilon^{\prime}}\left(1+\delta-\varepsilon^{\prime}\right)\right\} \\
& +[N \geq \delta] \sum_{r=\max }^{\llbracket \frac{N-\delta}{2} \rrbracket}\left\{\sum_{\left.a, 1+\llbracket \frac{N-\gamma}{2} \rrbracket\right\}}^{\delta-\varepsilon^{\prime}}(1+a)+\sum_{a=\delta-\varepsilon^{\prime}+1}^{N-2 r-\varepsilon^{\prime}}\left(1+\delta-\varepsilon^{\prime}\right)\right\} \\
& +\left[N \geq \varepsilon^{\prime}\right] \sum_{r=\max }^{\llbracket \frac{N-\varepsilon^{\prime}}{2} \rrbracket} \sum_{\left.0,1+\llbracket \frac{N-\delta}{2} \rrbracket\right\}}^{N-2 r-\varepsilon^{\prime}}(1+a) .
\end{aligned}
$$

In order to simplify further, we consider the various terms of (3.53) and (3.54) with 
the goal of writing everything in terms of $k_{0}=\min \{\gamma, \delta\}$. (Recall $\gamma+\delta=\varepsilon+\varepsilon^{\prime}$, so that $\left\{k_{0}, \varepsilon+\varepsilon^{\prime}-k_{0}\right\}=\{\gamma, \delta\}$.)

\section{Third term.}

The inner sum of the third term of (3.53) is

$$
\begin{aligned}
\sum_{a=N-2 r-\gamma}^{N-2 r-\varepsilon^{\prime}}(1+a) & =\sum_{a=0}^{\gamma-\varepsilon^{\prime}}(1+a+N-2 r-\gamma) \\
& =\sum_{a=0}^{\gamma-\varepsilon^{\prime}}(1+a)+(N-2 r-\gamma)\left(\gamma-\varepsilon^{\prime}+1\right) \\
& =\sum_{a=0}^{\gamma-\varepsilon^{\prime}}(1+a)+\sum_{a=\gamma-\varepsilon^{\prime}+1}^{N-2 r-\varepsilon^{\prime}}\left(1+\gamma-\varepsilon^{\prime}\right),
\end{aligned}
$$

which is the third term inner sum of (3.54) with $\gamma$ and $\delta$ interchanged. So the third term inner sum of both cases is

$$
\sum_{a=0}^{k_{0}-\varepsilon^{\prime}}(1+a)+\sum_{k_{0}-\varepsilon^{\prime}+1}^{N-2 r-\varepsilon^{\prime}}\left(1+k_{0}-\varepsilon^{\prime}\right) .
$$




\section{Second term.}

The inner sum of the second term of both (3.53) and (3.54) is

$$
\begin{aligned}
\sum_{a=N-2 r-\gamma}^{\delta-\varepsilon^{\prime}} & (1+a)+\sum_{a=\delta-\varepsilon^{\prime}+1}^{N-2 r-\varepsilon^{\prime}}\left(1+\delta-\varepsilon^{\prime}\right) \\
& =\sum_{a=N-2 r-\gamma+\varepsilon^{\prime}}^{\delta}\left(1+a-\varepsilon^{\prime}\right)+\sum_{a=\delta+1}^{N-2 r}\left(1+\delta-\varepsilon^{\prime}\right) \\
& =\sum_{a=N-2 r-\gamma+\varepsilon^{\prime}}^{N-2 r}-\sum_{a=\delta+1}^{N-2 r}\left(\left(1+a-\varepsilon^{\prime}\right)-\left(1+\delta-\varepsilon^{\prime}\right)\right) \\
& =\sum_{a=0}^{\gamma-\varepsilon^{\prime}}(1+a+N-2 r-\gamma)-\sum_{a=\delta+1}^{N-2 r}(a-\delta) \\
& =\sum_{a=0}^{\gamma-\varepsilon^{\prime}}(1+a)+(N-2 r-\gamma)\left(\gamma-\varepsilon^{\prime}+1\right)-\sum_{a=0}^{N-2 r-\delta-1}(1+a) \\
& =\sum_{a=N-2 r-\delta}^{\delta-\varepsilon^{\prime}}(1+a)+\sum_{a=\gamma-\varepsilon^{\prime}+1}^{N-2 r-\varepsilon^{\prime}}\left(1+\gamma-\varepsilon^{\prime}\right),
\end{aligned}
$$

which is symmetric in $\gamma$ and $\delta$, hence may be written

$$
\sum_{a=N-2 r-\varepsilon+k_{0}-\varepsilon^{\prime}}^{k_{0}-\varepsilon^{\prime}}(1+a)+\sum_{a=k_{0}-\varepsilon^{\prime}+1}^{N-2 r-\varepsilon^{\prime}}\left(1+k_{0}-\varepsilon^{\prime}\right) .
$$

First term.

It suffices to note that

$$
\begin{array}{r}
\sum_{a=0}^{\gamma-\varepsilon^{\prime}}\left(1+\delta-\varepsilon^{\prime}\right)=\left(1+\gamma-\varepsilon^{\prime}\right)\left(1+\delta-\varepsilon^{\prime}\right) \\
=\sum_{a=0}^{\delta-\varepsilon^{\prime}}\left(1+\gamma-\varepsilon^{\prime}\right)=\sum_{a=0}^{\varepsilon-k_{0}}\left(1+k_{0}-\varepsilon^{\prime}\right) .
\end{array}
$$


Now substituting (3.55),(3.56) and (3.57) into (3.53) (or (3.54)) yields

$$
\begin{aligned}
& D_{\mu, \nu, 2 N+M_{1}}=[N \geq \varepsilon] \sum_{r=0}^{\llbracket \frac{N-\varepsilon}{2} \rrbracket} \sum_{a=0}^{\varepsilon-k_{0}}\left(1+k_{0}-\varepsilon^{\prime}\right) \\
& +\left[N \geq \varepsilon-k_{0}\right] \sum_{r=\max \left\{0,1+\llbracket \frac{N-\varepsilon}{2} \rrbracket\right\}}^{\llbracket \frac{N-\varepsilon+k_{0}-\varepsilon^{\prime}}{2} \rrbracket}\left\{\sum_{\substack{a=N-2 r \\
-\varepsilon+k_{0}-\varepsilon^{\prime}}}^{k_{0}-\varepsilon^{\prime}}(1+a)+\sum_{k_{0}-\varepsilon^{\prime}+1}^{N-2 r-\varepsilon^{\prime}}\left(1+k_{0}-\varepsilon^{\prime}\right)\right\} \\
& +\left[N \geq k_{0}\right] \sum_{r=\max }^{\left.\llbracket 0,1+\llbracket \frac{N-\varepsilon-k_{0}-\varepsilon^{\prime}}{2} \rrbracket\right\}}\left\{\sum_{a=0}^{k_{0}-\varepsilon^{\prime}}(1+a)+\sum_{a=k_{0}-\varepsilon^{\prime}+1}^{N-2 r-\varepsilon^{\prime}}\left(1+k_{0}-\varepsilon^{\prime}\right)\right\} \\
& +\left[N \geq \varepsilon^{\prime}\right] \sum_{r=\max }^{\llbracket \frac{N-\varepsilon^{\prime}}{2} \rrbracket} \sum_{\left.0,1+\llbracket \frac{N-k_{0}}{2} \rrbracket\right\}}^{N-2 r-\varepsilon^{\prime}}(1+a) .
\end{aligned}
$$

Recall here that

$$
d_{a}= \begin{cases}1+a-\varepsilon^{\prime} & \text { if } \varepsilon^{\prime} \leq a \leq k_{0} \\ 1+k_{0}-\varepsilon^{\prime} & \text { if } k_{0} \leq a \leq \varepsilon-k_{0}+\varepsilon^{\prime} \\ 1+\varepsilon-a & \text { if } \varepsilon-k_{0}+\varepsilon^{\prime} \leq a \leq \varepsilon \\ 0 & \text { otherwise }\end{cases}
$$

so the last two terms are clearly just

$$
\left[N \geq \varepsilon^{\prime}\right] \sum_{r=\max \left\{0,1+\llbracket \frac{N-\varepsilon+k_{0}-\varepsilon^{\prime}}{2} \rrbracket\right\}}^{\llbracket \frac{N-\varepsilon^{\prime}}{2} \rrbracket} \sum_{a=\varepsilon^{\prime}}^{N-2 r} d_{a} .
$$

(We are using the fact that, for $r \in \mathbb{Z}, 1+\llbracket \frac{N-\alpha}{2} \rrbracket \leq r \leq \llbracket \frac{N-\beta}{2} \rrbracket$ if and only if $\beta \leq N-2 r \leq \alpha-1$.) 
Now the first term inner sum is

$$
\begin{aligned}
\sum_{a=0}^{\varepsilon-k_{0}}\left(1+k_{0}-\varepsilon^{\prime}\right)=\sum_{a-\varepsilon^{\prime}}^{k_{0}}\left(1+k_{0}-\varepsilon^{\prime}\right)+\sum_{a=k_{0}+1}^{\varepsilon-k_{0}+\varepsilon^{\prime}}\left(1+k_{0}-\varepsilon^{\prime}\right) \\
=\left(1+k_{0}-\varepsilon^{\prime}\right)^{2}+\sum_{a=k_{0}+1}^{\varepsilon-k_{0}+\varepsilon^{\prime}}\left(1+k_{0}-\varepsilon^{\prime}\right) \\
=\frac{1}{2}\left(1+k_{0}-\varepsilon^{\prime}\right)\left(2+k_{0}-\varepsilon^{\prime}\right)+\sum_{a=k_{0}+1}^{\varepsilon-k_{0}+\varepsilon^{\prime}}\left(1+k_{0}-\varepsilon^{\prime}\right) \\
\quad+\frac{1}{2}\left(k_{0}-\varepsilon^{\prime}\right)\left(1+k_{0}-\varepsilon^{\prime}\right) \\
=\sum_{a=\varepsilon^{\prime}}^{k_{0}}\left(1+a-\varepsilon^{\prime}\right)+\sum_{a=k_{0}+1}^{\varepsilon-k_{0}+\varepsilon^{\prime}}\left(1+k_{0}-\varepsilon^{\prime}\right)+\sum_{a=0}^{k_{0}-\varepsilon_{0}+\varepsilon^{\prime}}(1+a) \\
=\sum_{a=\varepsilon^{\prime}}^{k_{0}}\left(1+a-\varepsilon^{\prime}\right)+\sum_{a=k_{0}+1}^{\varepsilon}\left(1+k_{0}-\varepsilon^{\prime}\right)+\sum_{a=\varepsilon-k_{0}+\varepsilon^{\prime}+1}(1+\varepsilon-a) \\
=\sum_{a=\varepsilon^{\prime}}^{\varepsilon} d_{a} .
\end{aligned}
$$


The second term inner sum is

$$
\begin{aligned}
& \sum_{a=N-2 r-\varepsilon+k_{0}-\varepsilon^{\prime}}^{k_{0}-\varepsilon^{\prime}}(1+a)+\sum_{a=k_{0}-\varepsilon^{\prime}+1}^{N-2 r-\varepsilon^{\prime}}\left(1+k_{0}-\varepsilon^{\prime}\right) \\
& =\sum_{a=\varepsilon^{\prime}}^{k_{0}}\left(1+a-\varepsilon^{\prime}\right)-\sum_{a=\varepsilon^{\prime}}^{N-2 r-\varepsilon+k_{0}-1}\left(1+a-\varepsilon^{\prime}\right) \\
& \quad+\sum_{a=k_{0}+1}^{\varepsilon+\varepsilon^{\prime}-k_{0}}\left(1+k_{0}-\varepsilon^{\prime}\right)+\left(N-2 r-\varepsilon-\varepsilon^{\prime}+k_{0}\right)\left(1+k_{0}-\varepsilon^{\prime}\right) \\
& =\sum_{a=\varepsilon^{\prime}}^{k_{0}}\left(1+a-\varepsilon^{\prime}\right)+\sum_{a=k_{0}+1}^{\varepsilon+\varepsilon^{\prime}-k_{0}}\left(1+k_{0}-\varepsilon^{\prime}\right) \\
& \quad-\frac{1}{2}\left(N-2 r-\varepsilon-\varepsilon^{\prime}+k_{0}\right)\left(N-2 r-\varepsilon-\varepsilon^{\prime}+k_{0}+1\right) \\
& \quad+\left(N-2 r-\varepsilon-\varepsilon^{\prime}+k_{0}\right)\left(1+k_{0}-\varepsilon^{\prime}\right) \\
& =\sum_{a=\varepsilon^{\prime}}^{k_{0}}\left(1+a-\varepsilon^{\prime}\right)+\sum_{a=k_{0}+1}^{\varepsilon+\varepsilon^{\prime}-k_{0}}\left(1+k_{0}-\varepsilon^{\prime}\right) \\
& \quad+\frac{1}{2}\left(N-2 r-\varepsilon-\varepsilon^{\prime}+k_{0}\right)\left(1+k_{0}-\varepsilon^{\prime}-N+2 r+\varepsilon\right) .
\end{aligned}
$$

Now since

$$
\sum_{a=\alpha+1}^{\beta} a=\frac{1}{2} \beta(\beta+1)-\frac{1}{2} \alpha(\alpha+1)=\frac{1}{2}(\beta-\alpha)(\beta+\alpha+1)
$$

we have

$$
\begin{aligned}
& \sum_{a=\varepsilon+\varepsilon^{\prime}-k_{0}+1}^{N-2 r}(1+\varepsilon-a)=\sum_{\varepsilon-N+2 r+1}^{k_{0}-\varepsilon^{\prime}} a \\
& \quad=\frac{1}{2}\left(k_{0}-\varepsilon^{\prime}-\varepsilon+N-2 r\right)\left(k_{0}-\varepsilon^{\prime}+\varepsilon-N+2 r+1\right),
\end{aligned}
$$

which is the last term in (3.59). So the second term inner sum becomes

$$
\sum_{a=\varepsilon^{\prime}}^{k_{0}}\left(1+a-\varepsilon^{\prime}\right)+\sum_{a=k_{0}+1}^{\varepsilon+\varepsilon^{\prime}-k_{0}}\left(1+k_{0}-\varepsilon^{\prime}\right)+\sum_{a=\varepsilon+\varepsilon^{\prime}-k_{0}+1}^{N-2 r}(1+\varepsilon-a)=\sum_{a=\varepsilon^{\prime}}^{N-2 r} d_{a} .
$$


Combining all the above with (3.58) and dropping the initial $\left[N \geq \varepsilon^{\prime}\right]$ finally gives

$$
\begin{aligned}
& D_{\mu, \nu, 2 N+M_{1}}=\sum_{r=0} \llbracket \frac{N-\varepsilon^{\prime}}{2} \rrbracket \sum_{a=\varepsilon^{\prime}}^{\min \{\varepsilon, N-2 r\}} d_{a} \\
&=[N \geq \varepsilon] \sum_{r=0}^{\llbracket \frac{N-\varepsilon-1}{2} \rrbracket} \sum_{a=\varepsilon^{\prime}}^{\varepsilon} d_{a}+\sum_{r=\max \left\{0, \llbracket \frac{N-\varepsilon+1}{2} \rrbracket\right\}}^{\llbracket \frac{N-\varepsilon^{\prime}}{2} \rrbracket} \sum_{a=\varepsilon^{\prime}}^{N-2 r} d_{a} \\
&=[N \geq \varepsilon]\left(1+\llbracket \frac{N-\varepsilon-1}{2} \rrbracket\right) \sum_{a=\varepsilon^{\prime}}^{\varepsilon} d_{a}+\sum_{\varepsilon^{\prime} \leq a \leq N-2 r \leq \min \{\varepsilon, N\}} d_{a} \\
&=[N \geq \varepsilon] \llbracket \frac{N-\varepsilon+1}{2} \rrbracket \sum_{a=\varepsilon^{\prime}}^{\varepsilon} d_{a}+\sum_{a=\varepsilon^{\prime}}^{\min \{\varepsilon, N\}} d_{a} r: a \leq N-2 r \leq \min \{\varepsilon, N\} \\
&= {[N \geq \varepsilon] \llbracket \frac{N-\varepsilon+1}{2} \rrbracket \sum_{a=\varepsilon^{\prime}}^{\varepsilon} d_{a}+\sum_{a=\varepsilon^{\prime}}^{\min \{\varepsilon, N\}} d_{a}\left(\llbracket \frac{N-a}{2} \rrbracket-[N \geq \varepsilon] \llbracket \frac{N-\varepsilon+1}{2} \rrbracket+1\right) } \\
& \sum_{a=\varepsilon^{\prime}}^{\min \{\varepsilon, N\}} p_{2}(N-a) d_{a}, \quad[N]
\end{aligned}
$$

which is the result. 


\section{CHAPTER 4 ANALYSIS OF LOCAL INTEGRALS}

In order to complete our understanding of the twisted tensor $L$-function, we need more information about the local integrals appearing in (2.26). Specifically, we need to know that all the integrals are convergent in some region and nonvanishing. Moreover, in order to justify the absolute convergence of the Euler product, we need to establish a uniform line of convergence for the unramified integrals. This essentially depends on estimates on the unramified Whittaker functions.

Second, we would like to understand the location and order of the poles of our global $L$-function. In principle these should be the same as those of the global integral, but proving this is somewhat delicate. One must first show that the local integrals can be meromorphically continued, and then that the orders of poles of the local integrals are "no worse" than those of the corresponding local $L$-factor. We will say more about this in the closing remarks. As of now, these results are not included but are work in progress of the author.

Finally, we note that we will show the nonvanishing of the archimedean integrals for smooth data. Namely, for an archimedean place $v$, one can find a smooth section $f_{s, v}$ and a Whittaker function $W_{v}$ such that the integral $\Psi_{v}\left(s, W_{v}, f_{s, v}\right) \neq 0$. However, for the theory of Eisenstein series (and in particular for the results of [24]) one needs 
$K$-finite sections. So in order for our results here to be complete, we would need to show nonvanishing for $K$-finite data. For the moment, though, this disparity remains. By our arguments above, our local integral agrees with Jiang's local integral at all split places, so we will confine ourselves to inert and ramified places. (So if $F$ is archimedean, then we assume $F=\mathbb{R}$ and $E=\mathbb{C}$.) We should note that most of this material is a close parallel to, and at times a near-identical repetition of, corresponding material in [24], Chapter 5. Also, many of the ideas here are ultimately borrowed from or inspired by work in $[17,18,21,20]$, and the author greatly benefited from reading parts of [39].

Let $F$ be a local field of characteristic zero, and let $E / F$ be a quadratic extension generated by an element $\theta$ whose square lies in $F$. Let $\pi$ be an irreducible admissible generic unitary representation of $\operatorname{GSp}_{4}(E)$ with central character $\omega_{\pi}=1$. So $\pi$ has a Whittaker model, $\mathcal{W}(\pi, \psi)$, in the space $\mathcal{W}(\psi)$ of smooth complex-valued functions $W$ on $\operatorname{GSp}_{4}(E)$ satisfying $W(n g)=\psi(n) W(g)$ for $n \in N_{4}(E)$.

If $F$ is archimedean, we denote by $\mathcal{W}(\pi, \psi)_{K}$ the subspace of $K_{4}$-finite vectors of $\mathcal{W}(\pi, \psi)$, where $K_{4}$ is an appropriate maximal compact subgroup of $\mathrm{GSp}_{4}(\mathbb{C})$ (to be

defined below). Similarly denote the $K_{8}$-finite vectors of $I_{8}(s)=\operatorname{Ind}_{P_{8}(F)}^{\mathrm{GSp}_{8}(F)}\left(\delta_{P_{8}}^{s / 6}\right)$ by $I_{8}(s)_{K}$, with $K_{8}$ a maximal compact subgroup of $\mathrm{GSp}_{8}(\mathbb{R})$.

\subsection{Absolute Convergence}

We now give some properties of Whittaker functions on $\mathrm{GSp}_{4}$ which will be needed below. 
Recall that the root system of $\mathrm{GSp}_{4}$ has a basis of positive roots $\left\{\alpha_{1}=\epsilon_{1}-\epsilon_{2}, \alpha_{2}=\right.$ $\left.\epsilon_{2}-\epsilon_{4}\right\}$ as in Lemma 2.2.1. In (3.7) we fixed a parametrization of the quotient $Z(F) \backslash T_{0}(F)$, which we recall here:

$$
\begin{gathered}
t=t^{\prime}\left(a_{1}, a_{2}, b_{1}, b_{2}\right) \stackrel{\text { def }}{=} t\left(a_{1}\left(1+a_{2} \theta\right), b_{1}\left(1+b_{2} \theta\right), \lambda_{a, b}\left(1-a_{2} \theta\right), 1-b_{2} \theta\right), \\
\lambda_{a, b}=\frac{b_{1}\left(1-b_{2}^{2} \theta^{2}\right)}{a_{1}\left(1-a_{2}^{2} \theta^{2}\right)} \in F^{\times} .
\end{gathered}
$$

Note that

$$
t=t^{\prime}\left(a_{1}, a_{2}, b_{1}, b_{2}\right)=z \cdot h\left(A B, A, B^{-1}, 1\right), \quad z \in Z(E),
$$

where

$$
\begin{aligned}
& A=b_{1} \frac{1+b_{2} \theta}{1-b_{2} \theta}=\alpha_{1}(t), \\
& B=\frac{a_{1}\left(1+a_{2} \theta\right)}{b_{1}\left(1+b_{2} \theta\right)}=\alpha_{2}(t),
\end{aligned}
$$

and $h$ is defined by (3.1). We note for later reference that

$$
\delta_{B_{4}(E)}(t)=|A|^{3}|B|^{4} .
$$

Setting $a=1+a_{2} \theta, b=1+b_{2} \theta$ and $\mathbb{N} a=\mathrm{N}_{E / F} a, \mathbb{N} b=\mathrm{N}_{E / F} b$ as in Section 3.1 we see that

$$
\begin{aligned}
& |A|=\left|b_{1}\right|, \\
& |B|=\left|\frac{a_{1}}{b_{1}}\right|\left|\frac{\mathbb{N} a}{\mathbb{N} b}\right|^{1 / 2} .
\end{aligned}
$$

Since any Whittaker function $W \in \mathcal{W}(\pi, \psi)$ satisfies $W(z g)=\omega_{\pi}(z) W(g)=W(g)$, such a $W$ is determined by its restriction to the subgroup

$$
T_{4}(E)^{\prime}=\left\{h\left(a b, a, b^{-1}, 1\right) \mid a, b \in E^{\times}\right\} .
$$


Proposition 4.1.1. Let $F$ be a local field and $\pi$ an irreducible (admissible) generic representation of $\operatorname{GSp}_{4}(E)$ with Whittaker model $\mathcal{W}(\pi, \psi)$. There is a finite set $\mathbb{X}=$ $\mathbb{X}(\pi)$ of finite functions on $T_{4}(E)^{\prime}$, depending only on $\pi$, such that for $W \in \mathcal{W}(\pi, \psi)$ (K $K_{4}$-finite if $F$ is archimedean) there are Schwartz functions $\phi_{\xi} \in \mathcal{S}\left(F^{2}\right)$ satisfying

$$
W(t)=\sum_{\xi \in \mathbb{X}(\pi)} \xi\left(\alpha_{1}(t), \alpha_{2}(t)\right) \phi_{\xi}\left(\alpha_{1}(t), \alpha_{2}(t)\right) .
$$

Proof. For $F$ nonarchimedean, the argument is found in [17, 2.2], and its modification for $\mathrm{GSp}_{4}$ is in [24] in the discussion preceding Prop. V.1.1.1. For $F$ archimedean, see [24] and [20, 4.1-4.2].

In the archimedean case, we also have the following variant:

Proposition 4.1.2. Let $F$ be an archimedean local field and $\pi$ an irreducible (admissible) generic representation of $\mathrm{GSp}_{4}(E)$ with Whittaker model $\mathcal{W}(\pi, \psi)$. There is a finite set $\mathbb{X}=\mathbb{X}(\pi)$ of finite functions on $T_{4}(E)^{\prime}$, depending only on $\pi$, such that for $W \in \mathcal{W}(\pi, \psi)_{K}$ there are Schwartz functions $\phi_{\xi} \in \mathcal{S}\left(F^{2} \times K_{4}\right)$ satisfying

$$
W(t k)=\sum_{\xi \in \mathbb{X}(\pi)} \xi\left(\alpha_{1}(t), \alpha_{2}(t)\right) \phi_{\xi}\left(\left(\alpha_{1}(t), \alpha_{2}(t)\right), k\right) .
$$

Proof. See [20], Prop. 4.3.2.

For convergence of some auxiliary integrals, we will majorize our Whittaker functions by a gauge, a notion first used in $[17,2.2]$ and refined in $[21, \mathrm{p} .132]$ for $\mathrm{GL}_{n}$. We correspondingly define a gauge on $\mathrm{GSp}_{4}(E)$ to be a function $\xi$ which is left-invariant under $N_{4}(E) Z(E)$, right-invariant under $K_{4}$ and whose restriction to $T_{4}(E)^{\prime}$ satisfies

$$
\xi(t)=\sigma(t) \phi\left(\alpha_{1}(t), \alpha_{2}(t)\right)
$$


where $\sigma$ is a sum of positive quasicharacters of $T_{4}(E)$ and $\phi \in \mathcal{S}\left(F^{2}\right)$ is nonnegative. Since $\pi$ is generic, there is a nonzero linear functional $\Lambda: V_{\pi} \rightarrow \mathbb{C}$ such that $\Lambda(\pi(n) v)=\psi(n) \Lambda(v)$ for $n \in N_{4}(E), v \in V_{\pi}$. Set

$$
W_{v}(g)=\Lambda(\pi(g) v)
$$

Then $W_{v}$ is an element of the space $\mathcal{W}(\psi)$ of smooth functions on $\operatorname{GSp}_{4}(E)$ satisfying

$$
W(n g)=\psi(n) W(g), \quad n \in N_{4}(E), W \in \mathcal{W}(\psi),
$$

and the map $V_{\pi} \rightarrow \mathcal{W}(\psi)$ given by $v \mapsto W_{v}$ is an intertwining isomorphism from $\pi$ to a subspace of $\mathcal{W}(\psi)$, which we denote by $\mathcal{W}(\pi, \psi)$. Following arguments in [17], [18, 8.3] and [21], we arrive at

Proposition 4.1.3 (cf. [21], Prop. 2.1). Let $F$ be a nonarchimedean (archimedean) local field and $\pi$ an irreducible admissible generic representation of $\operatorname{GSp}_{4}(E)$. For any $f \in C_{c}^{\infty}(G)$ there is a gauge $\xi$ and a seminorm $p$ on $V_{\pi}$ such that $\left|W_{\pi(f) v}(g)\right| \leq$ $p(v) \xi(g)$, for all $v \in V_{\pi}$ (for all $K_{4}$-finite $\left.v \in V_{\pi}\right)$.

Proposition 4.1.4 ([15], Lem. 8.1). For F a local field, any finite (continuous) function on $F^{\times}$is a (finite) linear combination of functions of the form

$$
a \mapsto|a|^{u} \xi_{1}(a)(\log |a|)^{m}
$$

for $u \in \mathbb{R}, m \in \mathbb{Z}_{\geq 0}$ and $\xi_{1}$ a unitary character of $F^{\times}$.

We also need some basic information about a certain intertwining operator that appears in the course of evaluating the local integrals. Recall that in the unramified calculation we need to evaluate an integral of the form

$$
\int_{F^{3}} f_{s}(\chi(x, y, w, u, v)) \psi_{\circ}(\alpha x+\beta y) d x d y d w
$$


with $\psi_{\circ}$ an additive character of $F$ and $\chi(x, y, w, u, v)$ as in (3.3). We may write this as

$$
\chi(x, y, w, u, v)=\gamma_{0} n \bar{n}_{1},
$$

with

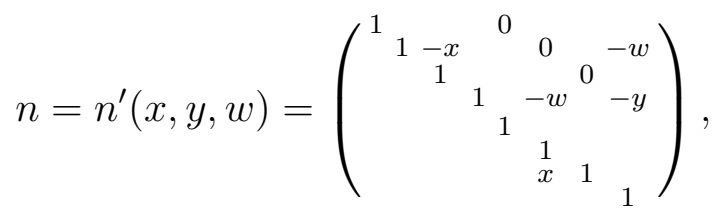

$$
\begin{aligned}
& \bar{n}_{1}=\bar{n}_{1}(u, v)=\left(\begin{array}{cccccc}
1 & & & & & \\
-u & 1 & & & & \\
& & 1 & & & \\
& -v & 1 & & & \\
& & & u & & \\
& & & & 1 & v \\
& & & & & 1
\end{array}\right) \text {. }
\end{aligned}
$$

Let $N_{8}^{\prime}(F)=\left\{n^{\prime}(x, y, w) \mid x, y, w \in F\right\}$.

Lemma 4.1.5 ([24], Lem. V.1.2.1). The integral

$$
M_{s} f_{s}(g)=\frac{\zeta_{v}(s+1)^{2}}{\zeta_{v}(s+2) \zeta_{v}(s+3)} \int_{N_{8}^{\prime}(F)} f_{s}\left(\gamma_{0} n g\right) d n
$$

defines an intertwining operator from $I_{8}(s)$ to $I_{B, \beta}(s)=\operatorname{Ind}_{B_{8}(F)}^{\mathrm{GSp}_{8}(F)}\left(\beta_{s}\right)$, where for $t=h\left(t_{1}, \ldots, t_{8}\right)$

$$
\beta_{s}(t)=\left|t_{1}\right|^{s+3}\left|t_{2}\right|^{2}\left|t_{3}\right|^{s+2}\left|t_{4}\right|^{-s}|\lambda|^{-\frac{s+7}{2}} .
$$

$M_{s}$ takes the unramified section $f_{s}^{\circ} \in I_{8}(s)$ to the unramified section $\tilde{f}_{s}^{\circ} \in I_{B, \beta}(s)$, where both are normalized by $f_{s}^{\circ}(1)=1=\tilde{f}_{s}^{\circ}(1)$.

Proof. All standard calculations.

Lemma 4.1.6. Let $f_{s} \in I_{8}(s)$ be a flat section and let $f_{s}^{\circ}$ and $\tilde{f}_{s}^{\circ}$ be the normalized unramified sections as in Lemma 4.1.5. 
(i). There is a constant $C_{f}$ which depends only on $\left.f_{s}\right|_{K}$ and such that

$$
\left|f_{s}(g)\right| \leq C_{f} f_{\sigma}^{\circ}(g), \quad \sigma=\operatorname{Re}(s) .
$$

(ii). For F nonarchimedean,

$$
\tilde{f}_{s}^{\circ}\left(\bar{n}_{1}(u, v)\right)=\max \left\{1,|u|^{s+1}\right\}^{-1} \max \left\{1,|v|^{2 s+2}\right\}^{-1} .
$$

(iii). For $F=\mathbb{R}$,

$$
\tilde{f}_{s}^{\circ}\left(\bar{n}_{1}(u, v)\right)=\left(1+u^{2}\right)^{-\frac{1}{2}(s+1)}\left(1+v^{2}\right)^{-(s+1)} .
$$

Proof. Standard computations.

\subsubsection{Nonarchimedean integrals}

Here we take $F$ to be nonarchimedean.

Proposition 4.1.7. There is a $\sigma_{0} \in \mathbb{R}$ such that for any section $f_{s}$ and any $W \in$ $\mathcal{W}(\pi, \psi)$, the integral $\Psi\left(s, W, f_{s}\right)$ is absolutely convergent for $\operatorname{Re}(s)>\sigma_{0}$.

Proof. As in Section 3.1 we have $G_{0}(F)=N_{0}(F) T_{0}(F) K_{0}$. For a subgroup $K \subseteq K_{0}$, let $\bar{K}=(K \cap Z(F)) \backslash K$. Our integral is

$$
\begin{aligned}
& \Psi\left(s, W, f_{s}\right)=\int_{Z(F) N^{Q}(F) X_{2}^{Q^{\prime}(F) \backslash G_{0}(F)}} f_{s}\left(\gamma_{0} g\right) W(g) d g \\
& \quad=\int_{\bar{K}_{0}} \int_{Z(F) \backslash T_{0}(F)} \int_{X_{2}^{Q^{\prime}}(F) N^{Q}(F) \backslash N_{0}(F)} f_{s}\left(\gamma_{0} n t k\right) W(n t k) \delta_{B_{0}}^{-1}(t) d n d t d k .
\end{aligned}
$$

Recall that $N_{0}(F)=N^{Q}(F) \cdot N^{Q^{\prime}}(F)$. Now choose an open compact subgroup $K^{\prime} \subseteq K_{0}$ such that both $W$ and $f_{s}$ are right- $K^{\prime}$-invariant, and write $K_{0}=\cup_{i=1}^{m} k_{i} K^{\prime}$. Then our integral is a sum of integrals

$$
\operatorname{vol}_{d \bar{k}}\left(K^{\prime}\right) \int_{Z(F) \backslash T_{0}(F)} \int_{X_{2}^{Q^{\prime}}(F) \backslash N Q^{\prime}(F)} f_{s, i}\left(\gamma_{0} n t\right) \psi(n) W_{i}(t) \delta_{B_{0}}^{-1}(t) d n d t,
$$


where $W_{i}=\pi\left(k_{i}\right) W \in \mathcal{W}(\pi, \psi)$ and $f_{s, i}=\pi\left(k_{i}\right) f_{s}$, and $d \bar{k}$ is the quotient measure on $\bar{K}_{0}$ normalized appropriately. So it is enough to show convergence of the integral

$$
\begin{aligned}
& \int_{Z(F) \backslash T_{0}(F)}\left|W(t) \delta_{B_{0}}^{-1}(t)\right| \int_{X_{2}^{Q^{\prime}}(F) \backslash N^{Q^{\prime}}(F)}\left|f_{s}\left(\gamma_{0} n t\right) \psi(n)\right| d n d t \\
\leq C_{f} & \int_{Z(F) \backslash T_{0}(F)}|W(t)| \delta_{B_{0}}^{-1}(t) \int_{X_{2}^{Q^{\prime}}(F) \backslash N^{Q^{\prime}}(F)} f_{\sigma}^{\circ}\left(\gamma_{0} n t\right) d n d t,
\end{aligned}
$$

where second line follows from (4.7) and the unitarity of $\psi$.

Now the inner integral is exactly $I\left(t, f_{\sigma}^{\circ}, 1\right)$ in the notation of Section 3.1, which was calculated in Remark 3.1.8. With $t=t^{\prime}\left(a_{1}, a_{2}, b_{1}, b_{2}\right)$ (see (4.1)), $a=1+a_{2} \theta$ and $b=1+b_{2} \theta,(3.9)$ gives

$$
I\left(t, f_{\sigma}^{\circ}, 1\right)=|\theta|^{-6}\left|a_{1}\right|^{\sigma+5}\left|b_{1}\right|^{-\frac{\sigma+3}{2}}|\mathbb{N} a|^{\frac{\sigma+5}{2}}|\mathbb{N} b|^{-\frac{\sigma+5}{2}} \frac{\zeta_{v}(\sigma+1)^{2}}{\zeta_{v}(\sigma+2) \zeta_{v}(\sigma+3)},
$$

which is defined and positive for $\sigma>-1$. Using (4.2) and (4.4), this becomes

$$
I\left(t, f_{\sigma}^{\circ}, 1\right)=|\theta|^{-6}|A|^{\frac{\sigma+7}{2}}|B|^{\sigma+5} \frac{\zeta_{v}(\sigma+1)^{2}}{\zeta_{v}(\sigma+2) \zeta_{v}(\sigma+3)} .
$$

We also replace $W(t)$ by its expansion (4.5):

$$
W(t)=\sum_{\xi \in \mathbb{X}(\pi)} \xi(A, B) \phi_{\xi}(A, B)
$$

By Prop. 4.1.4, $|\xi(A, B)|$ is bounded above by a finite linear combination of functions of the form

$$
|A|^{u_{1}}|B|^{u_{2}}|\log | A||^{m_{1}}|\log | B||^{m_{2}}, \quad u_{i} \in \mathbb{R}, m_{i} \in \mathbb{Z}_{\geq 0}
$$

The net effect is that $\Psi\left(s, W, f_{s}\right)$ is bounded above by a linear combination of integrals of the form

$$
\int_{\{0<|A|,|B|<\infty\}}|A|^{\frac{\sigma+1+2 u_{1}}{2}}|B|^{\sigma+1+u_{2}}|\log | A||^{m_{1}}|\log | B||^{m_{2}}|\phi(A, B)| d^{\times} A d^{\times} B .
$$


We have $|\log x| \leq C x^{\epsilon}$ for $x \geq 1$ and $|\log x| \leq C x^{-\epsilon}$ for $0<x \leq 1$, for any $\epsilon>0$ and some constant $C=C(\epsilon)$. Now since $\phi$ is compactly supported the integrand vanishes for $|A|$ and $|B|$ sufficiently large. So we may replace $|\log | A||^{m_{1}}$ and $|\log | B||^{m_{2}}$ by $|A|^{-\epsilon}$ and $|B|^{-\epsilon}$, respectively, for any $\epsilon>0$, for the purpose of proving convergence. Then the integral will converge near 0 when $\sigma$ is large enough, say $\sigma>\max \{-1-$ $\left.2 u_{1},-1-u_{2}\right\}$. So our integral $\Psi\left(s, W, f_{s}\right)$ also converges for $\sigma=\operatorname{Re}(s)$ large enough, being bounded above by a finite combination of such integrals. Finally we note that by our analysis a lower bound $\sigma_{0}$ for the line of convergence depends only on the finite functions $\chi \in \mathbb{X}(\pi)$, hence only on $\pi$ and not on the particular choice of $W$ or $f_{s}$.

\subsubsection{Archimedean integrals}

Let $K_{4}=\mathrm{Sp}_{4}(\mathbb{C}) \cap \mathrm{U}(4)$ denote the maximal compact subgroup of $\mathrm{GSp}_{4}(\mathbb{C})$ and $K_{8}=\operatorname{Sp}(8)=\operatorname{Sp}_{8}(\mathbb{R}) \cap \mathrm{O}_{8}(\mathbb{R})$ the maximal compact subgroup of $\mathrm{GSp}_{8}(\mathbb{R})$. The maximal compact subgroup $K_{0}$ in $G_{0}$ is

$$
K_{0}=\operatorname{Sp}(4)^{ \pm} \stackrel{\text { def }}{=} \operatorname{Sp}_{4}(\mathbb{C})^{ \pm} \cap \mathrm{U}(4) \stackrel{\text { def }}{=}\left\{g \in \mathrm{GL}_{4}(\mathbb{C}) \mid g J_{4}{ }^{t} g= \pm J_{4}, g^{t} \bar{g}=I_{4}\right\}
$$

For the decomposition $N_{4}(\mathbb{C})=N^{Q}(\mathbb{R}) N^{Q^{\prime}}(\mathbb{R})$, we may assume $\theta^{2}=-1$.

Proposition 4.1.8. There is a $\sigma_{0} \in \mathbb{R}$ such that for any smooth section $f_{s}$ and any $K_{4}$-finite Whittaker function $W \in \mathcal{W}(\pi, \psi)_{K}$, the integral $\Psi\left(s, W, f_{s}\right)$ is absolutely convergent for $\operatorname{Re}(s)>\sigma_{0}$. 
Proof. Using the Iwasawa decomposition just as in the nonarchimedean case, we have

$$
\begin{aligned}
& \Psi\left(s, W, f_{s}\right)=\int_{Z(F) N^{Q}(F) X_{2}^{Q^{\prime}}(F) \backslash G_{0}(F)} f_{s}\left(\gamma_{0} g\right) W(g) d g \\
& \quad=\int_{\bar{K}_{0}} \int_{Z(F) \backslash T_{0}(F)} \int_{X_{2}^{Q^{\prime}}(F) N^{Q}(F) \backslash N_{0}(F)} f_{s}\left(\gamma_{0} n t k\right) W(n t k) \delta_{B_{0}}^{-1}(t) d n d t d k,
\end{aligned}
$$

where $\bar{K}_{0}=\left(Z(F) \cap K_{0}\right) \backslash K_{0}$ as before. Given $W \in \mathcal{W}(\pi, \psi)_{K}$, we may write $\pi(k) W=\sum_{i=1}^{n} c_{i}(k) W_{i}$ for fixed $W_{i} \in \mathcal{W}(\pi, \psi)_{K}$ and continuous $K_{4}$-finite functions $c_{i}$. From this our integral becomes a sum

$$
\begin{gathered}
\sum_{i=1}^{n} \int_{Z(F) \backslash T_{0}(F)} W_{i}(t) \delta_{B_{0}}^{-1}(t) \int_{X_{2}^{Q^{\prime}}(F) N^{Q}(F) \backslash N_{0}(F)} \psi(n) \\
\cdot\left(\int_{\bar{K}_{0}} f_{s}\left(\gamma_{0} n t k\right) c_{i}(k) d k\right) d n d t .
\end{gathered}
$$

The integral over $\bar{K}_{0}$ defines a new section $f_{s, i}$ by

$$
f_{s, i}(g)=\int_{\bar{K}_{0}} f_{s}(g k) c_{i}(k) d k
$$

which is $K_{8}$-finite and also in $I_{8}(s)$. So it suffices to show the convergence of

$$
\int_{Z(F) \backslash T_{0}(F)}|W(t)| \delta_{B_{0}}^{-1}(t)\left(\int_{X_{2}^{Q^{\prime}}(F) N^{Q}(F) \backslash N_{0}(F)} f_{\sigma}^{\circ}\left(\gamma_{0} n t\right) d n\right) d t, \quad \sigma=\operatorname{Re}(s) .
$$

Now the inner integral is just $I\left(t, f_{\sigma}^{\circ}, 1\right)$ as in the nonarchimedean case. The proof of Lemma 3.1.2 carries over without change to the archimedean case; one only needs to check that $\gamma_{0} \in K_{8}$, and this is clear. So this fact together with Lemma 4.1.6 and Lemma 4.1.5 gives

$$
\begin{aligned}
I\left(t, f_{\sigma}^{\circ}, 1\right) & =\left|a_{1}\right|^{\sigma+5}\left|b_{1}\right|^{-\frac{\sigma+3}{2}}\left(1+a_{2}^{2}\right)^{\frac{\sigma+5}{2}}\left(1+b_{2}^{2}\right)^{-\frac{\sigma+5}{2}} \frac{\zeta_{v}(\sigma+1)^{2}}{\zeta_{v}(\sigma+2) \zeta_{v}(\sigma+3)} \\
& =|A|^{\frac{\sigma+7}{2}}|B|^{\sigma+5} c_{\sigma},
\end{aligned}
$$


where we have used $\mathbb{N} a=1+a_{2}^{2}, \mathbb{N} b=1+b_{2}^{2}$ and (4.2) and (4.4), with

$$
c_{\sigma}=\frac{\zeta_{v}(\sigma+1)^{2}}{\zeta_{v}(\sigma+2) \zeta_{v}(\sigma+3)}
$$

and as in $[43,3.1]$,

$$
\zeta_{v}(s)= \begin{cases}\pi^{-s / 2} \Gamma\left(\frac{s}{2}\right), & \text { if } F_{v}=\mathbb{R}, \\ 2(2 \pi)^{-s} \Gamma(s), & \text { if } F_{v}=\mathbb{C} .\end{cases}
$$

Now using Prop. 4.1.1 we reduce to showing convergence of integrals of the form

$$
\int_{\{0<|A|,|B|<\infty\}}|\xi(A, B)|\left|\phi_{\xi}(A, B)\right||A|^{-3}|B|^{-4} \cdot|A|^{\frac{\sigma+7}{2}}|B|^{\sigma+5} d^{\times} A d^{\times} B .
$$

As in the nonarchimedean case, using Prop. 4.1.4 shows this integral is bounded above by a finite linear combination of integrals of the form

$$
\int_{\{0<|A|,|B|<\infty\}}|A|^{\frac{\sigma+1+2 u_{1}}{2}}|B|^{\sigma+1+u_{2}}|\log | A||^{m_{1}}|\log | B||^{m_{2}}|\phi(A, B)| d^{\times} A d^{\times} B .
$$

Now estimating $\log |A|$ and $\log |B|$ as before and noting that the rapid decay of the Schwartz functions $\phi$ makes the integrals converge away from zero, we get that the integrals are convergent for $\sigma=\operatorname{Re}(s)$ large enough, say $\sigma>\max \left\{-1-2 u_{1},-1-u_{2}\right\}$. Then the convergence of $\Psi\left(s, W, f_{s}\right)$ independently of $W$ and $f_{s}$ follows as before.

\subsection{Nonvanishing}

Most of the results here are minor modifications of the analogous results in [24], Section V.2. 
Denote, for $x, z, w \in F$ and $g=\left(\begin{array}{ll}a & b \\ c & d\end{array}\right) \in \mathrm{GL}_{2}(F)$,

$$
\begin{aligned}
& m(g)=\left(\begin{array}{cccc}
e(g) & & & \\
& a & & -b \theta \\
& & 1 & \\
& -c \theta & & d
\end{array}\right) \in Q(F) \\
& e(g)=a d-b c \theta^{2} \in F^{\times}, \\
& u(x, z, w)=\left(\begin{array}{cccc}
1 & x & w \theta & z \theta \\
& 1 & z \theta & \\
& & 1 & \\
& & -x & 1
\end{array}\right) \in U^{Q}(F) \\
& u^{\prime}(x, z, w)=\left(\begin{array}{cccc}
1 & x(1+\theta) & w(1+\theta) & z \\
1 & z & \\
& 1 & & \\
& & -x(1+\theta) & 1
\end{array}\right) \in U^{Q^{\prime}}(F) \\
& u^{\prime \prime}(y)=\left(\begin{array}{cccc}
1 & & & \\
& 1 & & y(1-\theta) \\
& & & \\
& & & 1
\end{array}\right) \in N^{Q^{\prime}}(F) \\
& n(x, z, w)=\left(\begin{array}{cccc}
1 & x & w & z \\
& 1 & z & \\
& & 1 & \\
& & -x & 1
\end{array}\right) \in P_{4}(E) \text {, }
\end{aligned}
$$




$$
h(a, b, c, d)=\left(\begin{array}{llll}
a & & & \\
& b & & \\
& & & \\
& & & \\
& & & d
\end{array}\right) \in T_{4}(F) .
$$

Note that for $g \in B_{2}(F)$, the standard Borel subgroup of $\mathrm{GL}_{2}(F), e(g)=\operatorname{det} g$.

Let $C_{c}^{\infty}\left(F^{n}\right)$ be the space of smooth compactly supported functions on $F^{n}$; recall for $F$ nonarchimedean, smooth functions are just locally constant functions. It is easy to see that the linear map $\rho: C_{c}^{\infty}\left(U^{Q^{\prime}}(F)\right) \rightarrow C_{c}^{\infty}\left(F^{2}\right)$ given by

$$
\rho(\phi)(x, z)=\int_{F} \phi\left(u^{\prime}(x, z, w)\right) d w
$$

is well-defined and onto.

Let $\mathcal{S}\left(F^{n}\right)$ be the Schwarz space of smooth, rapidly decreasing functions on $F^{n}$, so for $F$ nonarchimedean $\mathcal{S}\left(F^{n}\right)=C_{c}^{\infty}\left(F^{n}\right)$. The Fourier transform defined on $L^{1}\left(F^{n}\right)$ restricts to an isomorphism $\mathcal{S}\left(F^{n}\right) \rightarrow \mathcal{S}\left(F^{n}\right)$. In particular, for the case $n=2$ we will need the Fourier transform $\Phi \mapsto \hat{\Phi}$ on $\mathcal{S}\left(F^{2}\right)$, given (with suitably normalized measures) by

$$
\begin{aligned}
& \hat{\Phi}(u, v)=\int_{F^{2}} \Phi(x, z) \psi_{\circ}\left(\theta^{2}(-u z-v x)\right) d x d z \\
& \Phi(x, z)=\int_{F^{2}} \hat{\Phi}(u, v) \psi_{\circ}\left(\theta^{2}(u z+v x)\right) d u d v
\end{aligned}
$$

which is an isomorphism $\mathcal{S}\left(F^{2}\right) \rightarrow \mathcal{S}\left(F^{2}\right)$.

To show the local integrals are nonzero, we will need a choice of section $f_{s} \in I_{8}(s)=$ $\operatorname{Ind}_{P_{8}(F)}^{\mathrm{GSS}_{8}(F)}\left(\delta_{P_{8}}^{s / 6}\right)$. Recall that $G_{0}(F)$ has two orbits on $P_{8}(F) \backslash \mathrm{GSp}_{8}(F)$, the open orbit 
$P_{8}(F) \gamma_{0} G_{0}(F)$ and the closed, negligible orbit $P_{8}(F) G_{0}(F)$. For sections supported on the open orbit (see (2.10)), we have an isomorphism of representations

$$
\left\{f \in I_{8}(s) \mid \operatorname{supp} f \subseteq P_{8} \gamma_{0} G_{0}\right\} \simeq \operatorname{ind}_{Q X_{2}^{Q^{\prime}}}^{G_{0}}\left(\Xi_{s}\right),
$$

where "ind" denotes non-normalized induction and $\Xi_{s}(x)=\delta_{P_{8}}\left(\gamma_{0} x \gamma_{0}^{-1}\right)^{\frac{s+3}{6}}$. The isomorphism is given by $f \mapsto f^{*}$, where $f^{*}(g)=f\left(\gamma_{0} g\right)$. A simple computation shows that for $g \in \mathrm{GL}_{2}(F), u(x, y, z) \in U^{Q}(F)$ and $h \in Z(F)$,

$$
\delta_{P_{8}}\left(\gamma_{0} m(g) \gamma_{0}^{-1}\right)=e(g)^{3},
$$

so that

$$
\Xi_{s}(m(g) u(x, z, w))=|e(g)|^{\frac{s+3}{2}}
$$

\subsubsection{Nonarchimedean case}

Let $v$ be a finite place of $F$ which is either inert or ramified. As usual we will drop the subscript $v$.

Recall that the integral under consideration is

$$
\Psi\left(s, W, f_{s}\right)=\int_{\mathcal{D}(F)} f_{s}\left(\gamma_{0} g\right) W(g) d g .
$$

The main result here is the following.

Proposition 4.2.1. Let $\sigma_{0}$ be as in Prop. 4.1.7. Then the integral $\Psi\left(s, W, f_{s}\right)$ is not identically zero for $\operatorname{Re}(s)>\sigma_{0}$. More precisely, there is a choice of Whittaker function $W_{0}$ and section $f_{s, 0}$ such that $\Psi\left(s, W_{0}, f_{s, 0}\right)=1$, independent of $s$, for $\operatorname{Re}(s)>\sigma_{0}$. 
This will be accomplished through several reductions.

Let $P_{2}^{\prime}$ be the standard mirabolic subgroup of $\mathrm{GL}_{2}$ with unipotent radical $N_{2}$, so $\mathrm{GL}_{2}(F)=P_{2}^{\prime}(F) Z(F) K_{2}$, with $K_{2}=\mathrm{GL}_{2}(\mathcal{O})$. For a Whittaker function $\widetilde{W}$ and $g \in \mathrm{GL}_{2}(F)$, consider the integral

$$
J(s, g, \widetilde{W})=\int_{N_{2}(F) \backslash P_{2}^{\prime}(F)}|\operatorname{det} p|^{s} \widetilde{W}(m(p g)) d p .
$$

We note that $J(s, p g, \widetilde{W})=J(s, g, \widetilde{W})$ for $p \in P_{2}^{\prime}(F) \cap K_{2}$, and $J(s, g, \widetilde{W})$ is absolutely convergent for $\operatorname{Re}(s)$ large enough by Prop. 4.1.3.

Lemma 4.2.2. The integral $J(s, g, \widetilde{W})$ defines a smooth function of $g$, and we may choose $\widetilde{W}$ so that $J(s, 1, \widetilde{W})$ is a nonzero constant independent of $s$, for $\operatorname{Re}(s)$ sufficiently large.

Proof. The smoothness in $g$ is clear from that of the Whittaker function $\widetilde{W}$. Choose $\widetilde{W^{\prime}} \in \mathcal{W}(\pi, \psi)$ such that $\widetilde{W^{\prime}}(1)=1$, and set

$$
\widetilde{W}(g)=\int_{F} \widetilde{W^{\prime}}\left(g u^{\prime \prime}(y)\right) \hat{\Phi}^{\prime}(y) d y
$$

with $\Phi^{\prime} \in C_{c}^{\infty}(F)$ to be chosen below, and with Fourier transform given by

$$
\hat{\Phi}(y)=\int_{F} \Phi(x) \psi_{\circ}(x y)^{-1} d x .
$$

Then

$$
\begin{aligned}
J(s, 1, \widetilde{W}) & =\int_{N_{2}(F) \backslash P_{2}^{\prime}(F)}|\operatorname{det} p|^{s} \widetilde{W}(m(p)) d p \\
& =\int_{F^{\times}}|a|^{s} \widetilde{W}(h(a, a, 1,1)) d^{\times} a \\
& =\int_{F^{\times}}|a|^{s} \int_{F} \widetilde{W^{\prime}}\left(h(a, a, 1,1) u^{\prime \prime}(y)\right) \hat{\Phi}^{\prime}(y) d y d^{\times} a \\
& =\int_{F^{\times}}|a|^{s} \widetilde{W^{\prime}}(h(a, a, 1,1)) \int_{F} \psi\left(u^{\prime \prime}(a y)\right) \hat{\Phi}^{\prime}(y) d y d^{\times} a .
\end{aligned}
$$


Now since $\psi\left(u^{\prime \prime}(a y)\right)=\psi_{\circ}\left(\frac{1}{2} \operatorname{Tr}(a y(1-\theta))\right)=\psi_{\circ}(a y)$, the inner integral is

$$
\int_{F} \hat{\Phi}^{\prime}(y) \psi_{\circ}(a y) d y=\Phi^{\prime}(a)
$$

SO

$$
J(s, 1, \widetilde{W})=\int_{F^{\times}}|a|^{s} \widetilde{W^{\prime}}(h(a, a, 1,1)) \Phi^{\prime}(a) d^{\times} a .
$$

Now take $\Phi^{\prime} \in C_{c}^{\infty}(F)$ nonnegative and with support containing 1 and small enough so that the last integrand is the positive constant $\widetilde{W}^{\prime}(1)=1$ on the support of $\Phi^{\prime}$. This choice of $\Phi^{\prime}$ depends only on $\widetilde{W^{\prime}}$, not on $s$, and we may scale $\Phi^{\prime}$ (or $\widetilde{W^{\prime}}$ ) so the integral is equal to 1 , when $s$ is in the domain of convergence.

Next, let $\Phi$ be a Schwarz-Bruhat function on $F^{2}$ and consider the integral

$$
I_{1}(s, \widetilde{W}, \Phi)=\int_{N_{2}(F) \backslash \mathrm{GL}_{2}(F)}|e(g)|^{s} \widetilde{W}(m(g)) \Phi((0,1) g) d g .
$$

This also converges for $\operatorname{Re}(s)>>0$ by Prop. 4.1.3.

Lemma 4.2.3. We may choose $\Phi$ and $\widetilde{W}$ so that $I_{1}(s, \widetilde{W}, \Phi)$ is a nonzero constant independent of $s$, for $\operatorname{Re}(s)$ sufficiently large.

Proof. Using the decomposition $N_{2} \backslash \mathrm{GL}_{2}=\left(N_{2} \backslash P_{2}^{\prime}\right) Z_{2} K_{2}$, we may write the integral as

$$
\begin{aligned}
& I_{1}(s, \widetilde{W}, \Phi)=\int_{N_{2}(F) \backslash P_{2}^{\prime}(F)} \int_{Z_{2}(F)} \int_{K_{2}}|e(p z k)|^{s} \delta_{B_{2}}^{-1}(p z) \\
& \cdot \widetilde{W}(m(p z k)) \Phi((0,1) p z k) d k d z d p \\
& =\int_{K_{2}} \int_{Z_{2}(F)} \Phi((0,1) z k)|e(z)|^{s}\left(\int_{N_{2}(F) \backslash P_{2}^{\prime}(F)}|e(p)|^{s-1} \widetilde{W}(m(p z k)) d p\right) d z d k \\
& =\int_{K_{2}} \int_{Z_{2}(F)} \Phi((0,1) z k)|e(z)|^{s} J(s-1, z k, \widetilde{W}) d z d k .
\end{aligned}
$$


Now by Lemma 4.2.2, $J\left(s^{\prime}, g, \widetilde{W}\right)$ is smooth in $g$ and nonvanishing at $g=1$, so there is an open compact subgroup $K_{2}^{\prime} \subseteq K_{2}$ so that $\left.J\right|_{K_{2}^{\prime}}$ is a constant, say 1 . In fact, we may choose $K_{2}^{\prime}$ of the form

$$
K_{2}^{\prime}=\left\{k \in K_{2} \mid k \equiv I_{2} \quad\left(\bmod \mathfrak{p}^{\ell}\right)\right\}=\left\{\left(\begin{array}{cc}
1+\mathfrak{p}^{\ell} & \mathfrak{p}^{\ell} \\
\mathfrak{p}^{\ell} & 1+\mathfrak{p}^{\ell}
\end{array}\right)\right\}, \quad \text { some } \ell \geq 0
$$

with $\mathfrak{p}=\varpi \mathcal{O}$ the maximal ideal in $\mathcal{O}$, so that $(0,1) K_{2}^{\prime} \subseteq\left(\mathfrak{p}^{\ell}, 1+\mathfrak{p}^{\ell}\right)$. Set $\Phi=\mathbf{1}_{(0,1) K_{2}^{\prime}}$. Note that for $z=\left(\begin{array}{c}a \\ a\end{array}\right)$ and $k=\left(k_{i j}\right) \in K_{2}$, either $k_{21}$ or $k_{22}$ is a unit, so if $z k \in K_{2}^{\prime}$ then $(0,1) z k=\left(a k_{21}, a k_{22}\right) \in\left(\mathfrak{p}^{\ell}, 1+\mathfrak{p}^{\ell}\right)$. If $k_{22} \in \mathcal{O}^{\times}$, then $a k_{22} \in 1+\mathfrak{p}^{\ell} \subseteq \mathcal{O}^{\times}$ implies $a \in \mathcal{O}^{\times}$. If $k_{21} \in \mathcal{O}^{\times}$, then $a k_{21} \in \mathfrak{p}^{\ell}$ implies $a \in \mathfrak{p}^{\ell}$, but then $k_{22} \in \mathcal{O}$ forces $a k_{22} \in \mathfrak{p}^{\ell}$, a contradiction. Thus $a \in \mathcal{O}^{\times}$. Also, if $\Phi((0,1) k) \neq 0$ for $k \in K_{2}$, then $k=p k^{\prime}$ for $k^{\prime} \in K_{2}^{\prime}$ and $p=k k^{\prime-1} \in P_{2}^{\prime}(F) \cap K_{2}$. By [27], Thm. 8.32 and its proof (noting that in this case both $S=P_{2}^{\prime}(F) \cap K_{2}$ and $T=K_{2}^{\prime}$ are compact), we can decompose the integral as

$$
\begin{aligned}
I_{1} & (s, \widetilde{W}, \Phi)=\int_{K_{2}} \Phi((0,1) k) J(s-1, k, \widetilde{W}) d k \\
& =\int_{K_{2}^{\prime}} \int_{P_{2}^{\prime}(F) \cap K_{2}} J(s-1, p k, \widetilde{W}) d p d k^{\prime} \\
& =\operatorname{vol}_{d p}\left(P_{2}^{\prime}(F) \cap K_{2}\right) \int_{K_{2}^{\prime}} J(s-1, k, \widetilde{W}) d k^{\prime} \\
& =\operatorname{vol}_{d p}\left(P_{2}^{\prime}(F) \cap K_{2}\right) \operatorname{vol}_{d k^{\prime}}\left(K_{2}^{\prime}\right) \neq 0 .
\end{aligned}
$$

Proof of Proposition 4.2.1. With $\widetilde{W}$ as in Lemma 4.2.3, define

$$
W_{0}(g)=\int_{U^{Q^{\prime}(F)}} \widetilde{W}\left(g u^{\prime}\right) \phi\left(u^{\prime}\right) d u^{\prime}
$$

where $\phi \in C_{c}^{\infty}\left(U^{Q^{\prime}}(F)\right)$ is such that $\rho(\phi)=\hat{\Phi}$, with $\Phi$ as in Lemma 4.2.3. Then $W_{0}$ is 
another Whittaker function which is right-invariant by some open compact subgroup $K_{0}^{\prime}$ of $K_{0}$.

Claim. We can choose $f^{*} \in \operatorname{ind}_{Q X_{2}^{Q^{\prime}}}^{G_{0}}\left(\Xi_{s}\right)$ with supp $f^{*} \subseteq\left(Q X_{2}^{Q^{\prime}}\right)(F) \cdot K_{0}^{\prime}$, such that $f^{*}$ restricted to $K_{0}^{\prime}$ is a nonzero constant.

We will prove this below. Assuming it for now, we proceed to the integral, taking $f_{s, 0}$ to correspond to $f^{*}$ under (4.12):

$$
\begin{aligned}
\Psi\left(s, W_{0}, f_{s, 0}\right) & =\int_{Z(F) N^{Q}(F) X_{2}^{Q^{\prime}}(F) \backslash G_{0}(F)} f_{s, 0}\left(\gamma_{0} g\right) W_{0}(g) d g \\
& =\int_{Z(F) N^{Q}(F) X_{2}^{Q^{\prime}}(F) \backslash Q(F) X_{2}^{Q^{\prime}}(F) K_{0}^{\prime}} f^{*}(g) W_{0}(g) d g \\
& =\int_{Z(F) N^{Q}(F) \backslash Q(F)} \int_{\widetilde{K}_{0}^{\prime}} f^{*}\left(q k^{\prime}\right) W_{0}\left(q k^{\prime}\right) d k^{\prime} d q \\
& =\int_{Z(F) N^{Q}(F) \backslash Q(F)} \Xi_{s}(q) W_{0}(q) \int_{\widetilde{K}_{0}^{\prime}} f^{*}\left(k^{\prime}\right) d k^{\prime} d q,
\end{aligned}
$$

where $\widetilde{K}_{0}^{\prime}=\left(K_{0}^{\prime} \cap Z(F) N^{Q}(F) X_{2}^{Q^{\prime}}(F)\right) \backslash K_{0}^{\prime}$. So the inner integral is a constant $C=C_{f^{*}} \neq 0$, and since $Z(F) N^{Q}(F) \backslash Q(F) \simeq N_{2}(F) \backslash \mathrm{GL}_{2}(F)$, this becomes

$$
\begin{aligned}
& \Psi\left(s, W_{0}, f_{s, 0}\right)=C \int_{N_{2}(F) \backslash \mathrm{GL}_{2}(F)}|e(g)|^{\frac{s+3}{2}} W_{0}(m(g)) d g \\
& \quad=C \int_{N_{2}(F) \backslash \mathrm{GL}_{2}(F)}|e(g)|^{s^{\prime}} \int_{U^{Q^{\prime}}(F)} \widetilde{W}\left(m(g) u^{\prime}\right) \phi\left(u^{\prime}\right) d u^{\prime} d g \\
& \quad=C \int_{N_{2}(F) \backslash \mathrm{GL}_{2}(F)}|e(g)|^{s^{\prime}} \widetilde{W}(m(g)) \int_{U^{Q^{\prime}(F)}} \phi\left(u^{\prime}\right) \psi\left(m(g) u^{\prime} m(g)^{-1}\right) d u d g
\end{aligned}
$$


with $s^{\prime}=\frac{s+3}{2}$. Now for $g=\left(\begin{array}{ll}a & b \\ c & d\end{array}\right), m(g) u^{\prime}(x, z, w) m(g)^{-1}=n\left(x_{1}, z_{1}, w_{1}\right)$, where

$$
\begin{aligned}
& x_{1}=c z \theta+d x(1+\theta), \\
& z_{1}=a z+b x \theta(1+\theta), \\
& w_{1}=e(g) w(1+\theta),
\end{aligned}
$$

so

$$
\psi\left(m(g) u^{\prime}(x, z, w) m(g)^{-1}\right)=\psi_{\circ}\left(\theta^{2}(c z+d x)\right) .
$$

Hence, by our choice of $\phi$, the inner integral is

$$
\begin{aligned}
\int_{F^{2}}\left(\int_{F} \phi\right. & \left.\left(u^{\prime}(x, z, w)\right) d w\right) \psi_{\circ}\left(\theta^{2}(c z+d x)\right) d x d z \\
& =\int_{F^{2}} \rho(\phi)(x, z) \psi_{\circ}\left(\theta^{2}(c z+d x)\right) d x d z \\
& =\int_{F^{2}} \hat{\Phi}(x, z) \psi_{\circ}\left(\theta^{2}(c z+d x)\right) d x d z \\
& =\Phi(c, d)=\Phi((0,1) g) . \quad \quad \quad \text { (since }(c, d) \neq(0,0))
\end{aligned}
$$

Putting all this together yields

$$
\Psi\left(s, W_{0}, f_{s, 0}\right)=C \int_{N_{2}(F) \backslash \mathrm{GL}_{2}(F)}|e(g)|^{s^{\prime}} \widetilde{W}(m(g)) \Phi((0,1) g) d g
$$

By Lemma 4.2.3, this is a nonzero constant independent of $s^{\prime}$, so we are done.

Now we prove the Claim. Let $Y$ be the root subgroup of $\mathrm{GSp}_{4}$ for the root $-2 \epsilon_{1}$, so 
that $Y \cdot B_{4}=P_{4}$, and let $Y^{Q}(F)=Q(F) \cap Y(E)$. Then

$$
\begin{aligned}
G_{0}(F) & =Y^{Q}(F) B_{0}(F) K_{0} \\
& =Y^{Q}(F) N^{Q}(F) N^{Q^{\prime}}(F) T_{4}(E)^{\circ} K_{0} \\
& =Y^{Q}(F) N^{Q}(F) T_{4}(F) N^{Q^{\prime}}(F) \widetilde{T} K_{0} \\
& =Q(F) N^{Q^{\prime}}(F) \widetilde{T} K_{0} \\
& =\left(Q X_{2}^{Q^{\prime}}\right)(F) \cdot \widetilde{N^{Q^{\prime}}}(F) \widetilde{T} K_{0},
\end{aligned}
$$

where

$$
\widetilde{T}=\left\{\begin{array}{cc}
\left(\mathcal{O}_{F}^{\times} \backslash \mathcal{O}_{E}^{\times}\right)^{2} & \text { if } E / F \text { is unramified, } \\
\left\{t_{\epsilon_{1}, \epsilon_{2}} \mid \epsilon_{1}, \epsilon_{2} \in\{0,1\}\right\} \cdot\left(\mathcal{O}_{F}^{\times} \backslash \mathcal{O}_{E}^{\times}\right)^{2} \\
\text { if } E / F \text { is ramified }
\end{array}\right\} \simeq T_{4}(F) \backslash T_{4}(E)^{\circ}
$$

with

$$
t_{\epsilon_{1}, \epsilon_{2}}=\left(\begin{array}{cccc}
\theta^{\epsilon_{1}} & & & \\
& \theta^{\epsilon_{2}} & & \\
& & \theta^{-\epsilon_{1}} & \\
& & & \theta^{-\epsilon_{2}}
\end{array}\right)
$$

and

$$
\widetilde{N}^{Q^{\prime}}(F)(F)=\left\{u^{\prime \prime}(y) u^{\prime}(x, z, 0) \mid x, y, z \in F\right\} \subseteq N^{Q^{\prime}}(F)
$$

Note that $\tilde{N}^{Q^{\prime}}(F)$ is a subset (!) which clearly commutes with $X_{2}^{Q^{\prime}}(F)$, satisfies $X_{2}^{Q^{\prime}}(F) \cdot \tilde{N}^{Q^{\prime}}(F)=N^{Q^{\prime}}(F)$ and is mapped bijectively onto $X_{2}^{Q^{\prime}}(F) \backslash N^{Q^{\prime}}(F)$ under the quotient map.

Also, in decomposing $N_{4}(E)=N^{Q}(F) \cdot N^{Q^{\prime}}(F)$, we may replace $\theta$ by $\varpi_{F}^{*} \theta$ and so assume $\theta \in \mathcal{O}_{E}^{\times}$(in the unramified case) or $\theta \in \varpi_{E} \mathcal{O}_{E}^{\times}$(in the ramified case). In particular, $\widetilde{T} K_{0}$ is either $K_{0}$ or $(\mathbb{Z} / 2 \mathbb{Z})^{2} \cdot K_{0}$. 
To define $f^{*}$, we need to understand the intersection $\left(Q X_{2}^{Q^{\prime}}\right)(F) \cap \widetilde{N}^{Q^{\prime}}(F) \widetilde{T} K_{0}$. For this we have a

Lemma 4.2.4. Let $\epsilon_{1}, \epsilon_{2} \in\{0,1\}$ and let $\epsilon_{\epsilon_{1}, \epsilon_{2}}$ be as in (4.21), noting that $\epsilon_{1}=0=\epsilon_{2}$ if $E / F$ is unramified.

(i). The sets $\widetilde{N}^{Q^{\prime}}(F) t_{\epsilon_{1}, \epsilon_{2}} K_{0}$ are disjoint for distinct pairs $\left(\epsilon_{1}, \epsilon_{2}\right)$.

(ii). $\left(Q X_{2}^{Q^{\prime}}\right)(F) \cap \widetilde{N}^{Q^{\prime}}(F) t_{\epsilon_{1}, \epsilon_{2}} K_{0}=\emptyset$ unless $\epsilon_{1}=0$.

(iii). $\left(Q X_{2}^{Q^{\prime}}\right)(F) \cap \widetilde{N}^{Q^{\prime}}(F) t_{0,0} K_{0} \subseteq\left(Q X_{2}^{Q^{\prime}}\right)(F) \cap K_{0}$.

(iv). $\left(Q X_{2}^{Q^{\prime}}\right)(F) \cap \widetilde{N}^{Q^{\prime}}(F) t_{0,1} K_{0} \subseteq\left(Q X_{2}^{Q^{\prime}}\right)(F) \cap t_{\theta} K_{0} t_{\theta}^{-1}$, for some $t_{\theta} \in T_{4}(E)$.

Proof. Recall that $Q(F)$ has Levi decomposition

$$
Q(F)=M^{Q}(F) U^{Q}(F)=Z(F) \widetilde{M}^{Q}(F) U^{Q}(F),
$$

with $\widetilde{M}^{Q}$ as in (2.11), and similarly for $P_{4}(E)$. We will make use of the isomorphisms given by

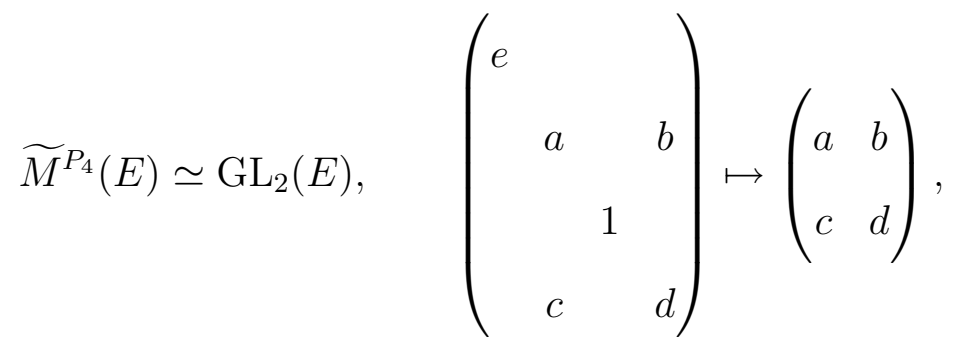

$$
\begin{aligned}
& X_{2}^{P_{4}}(E) \backslash U^{P_{4}}(E) \simeq E^{2}, \quad \overline{\left(\begin{array}{cccc}
1 & x & w & s \\
& 1 & s & \\
& & 1 & \\
& & -x & 1
\end{array}\right)} \mapsto\left(\begin{array}{c}
s \\
-x
\end{array}\right),
\end{aligned}
$$


respectively. Note that the (left) action of $\widetilde{M}^{P_{4}}(E)$ on $U^{P_{4}}(E)$ by conjugation is transferred to left multiplication by $\mathrm{GL}_{2}(E)$ on $E^{2}$ under these isomorphisms.

(i). Let $t_{1}=t_{\epsilon_{1}, \epsilon_{2}}$ and $t_{2}=t_{\epsilon_{3}, \epsilon_{4}}$ and suppose $\widetilde{N}^{Q^{\prime}}(F) t_{1} K_{0} \cap \widetilde{N}^{Q^{\prime}}(F) t_{2} K_{0}$ is nonempty. Then there is an $n^{\prime} \in N^{Q^{\prime}}(F)$ such that $t_{2}^{-1} n^{\prime} t_{1}=\left(t_{2}^{-1} n^{\prime} t_{2}\right) t_{2}^{-1} t_{1} \in K_{0}$. But this implies $t_{2}^{-1} t_{1} \in K_{0}$, which can only happen if $\epsilon_{1}=\epsilon_{3}$ and $\epsilon_{2}=\epsilon_{4}$, so $t_{1}=t_{2}$.

(ii). Let $g=q v=q^{\prime} t k \in\left(Q X_{2}^{Q^{\prime}}\right)(F) \cap \tilde{N}^{Q^{\prime}}(F) t_{\epsilon_{1}, \epsilon_{2}} K_{0}$, with $t=t_{\epsilon_{1}, \epsilon_{2}}$. Then $k=t^{-1} q^{-1} q v \in P_{4}(E)$, so we have Levi decompositions $k=z_{k} m_{k} n_{k}, q=z m n$ and $q^{\prime}=m^{\prime} n^{\prime}$ where $m^{\prime}=u^{\prime \prime}(y)$ and $n^{\prime}=u^{\prime}\left(x^{\prime}, s^{\prime}, 0\right)$. Then

$$
z m n v=m^{\prime} n^{\prime} t z_{k} m_{k} n_{k}
$$

implies equality of Levi factors, $z m=z_{k} m^{\prime} t m_{k}$. Noting that $e^{\prime}(m)=e^{\prime}\left(m_{k}\right)=$ $e^{\prime}\left(m^{\prime}\right)=1$, we get $e^{\prime}(z)=e^{\prime}\left(z_{k}\right) e^{\prime}(t)$ and hence $\theta^{\epsilon_{1}}=e^{\prime}(t)^{-1}=e^{\prime}\left(z_{k}\right) e^{\prime}(z)^{-1} \in$ $F^{\times} \cdot \mathcal{O}_{E}^{\times}$, which means $\epsilon_{1}=0$.

(iii). Taking $t=t_{0,0}=1$ in the previous argument, we see $e^{\prime}(z)=e^{\prime}\left(z_{k}\right) \in F^{\times} \cap \mathcal{O}_{E}^{\times}=$ $\mathcal{O}_{F}^{\times}$, so $z=z_{k} \in Z(F) \cap K_{0}$. Using uniqueness of Levi decomposition in (4.24) we see that

$$
\begin{aligned}
m & =m^{\prime} m_{k} \\
n v & =m_{k}^{-1} n^{\prime} m_{k} n_{k} .
\end{aligned}
$$

First let us show that $m, m^{\prime} \in K_{0}$. By our choice of $m \in \widetilde{M}^{Q}(F)$ and $m^{\prime}=$ $u^{\prime \prime}(y) \in \widetilde{M}^{Q^{\prime}}(F)$ and using (4.23), it is enough to show that

$$
g=g^{\prime} g_{k} \quad \Longrightarrow \quad g, g^{\prime} \in \mathrm{GL}_{2}\left(\mathcal{O}_{E}\right)
$$


for

$$
\begin{aligned}
& g=\left(\begin{array}{cc}
a & b \theta \\
c \theta & d
\end{array}\right) \in \mathrm{GL}_{2}(E), \quad(a, b, c, d \in F) \\
& g^{\prime}=\left(\begin{array}{cc}
1 & y(1-\theta) \\
1
\end{array}\right), \quad(y \in F) \\
& g_{k}=\left(\begin{array}{cc}
\alpha & \beta \\
\gamma & \delta
\end{array}\right) \in \mathrm{GL}_{2}\left(\mathcal{O}_{E}\right)
\end{aligned}
$$

This is a matter of checking entry-by-entry, and noting that if $\alpha=\alpha_{1}+\alpha_{2} \theta \in$ $\mathcal{O}_{E}$, then both $\alpha_{1}, \alpha_{2} \in \mathcal{O}_{F}$ since $\operatorname{ord}_{E} \theta=1$ and $\operatorname{ord}_{E} a$ is even for any $a \in F$. Now since $m_{k}=m^{\prime-1} m$ by (4.25), we may rewrite (4.26) as

$$
n v=m^{-1} m^{\prime} n^{\prime} m^{\prime-1} m n_{k}=m^{-1} n_{1}^{\prime} m n_{k}, \quad n_{1}^{\prime} \in N^{Q^{\prime}}(F) .
$$

Since $m^{\prime} \in K_{0}$, we have $n^{\prime} \in K_{0}$ if and only if $n_{1}^{\prime} \in K_{0}$, so we may assume for our purpose that $n^{\prime}=n_{1}^{\prime}$. Since $n^{\prime} \in \widetilde{N}^{Q^{\prime}}(F)$, the $X_{2}$-entry of $m^{-1} n^{\prime} m n_{k}$ is in $\mathcal{O}_{E}$ and is equal to the $X_{2}$-entry of $v$ (which is in $F(1+\theta) \subseteq E$ ) plus the $X_{2}$-entry of $n$ (which is in $F \theta \subseteq E$ ), which implies $v \in K_{0}$ and the $X_{2}$-entry of $n$ is in $\mathcal{O}_{E}$. So we must show $n=m^{-1} n^{\prime} m n_{k}$ implies $n, n^{\prime} \in K_{0}$. Using (4.23) with $n=u(x, s, w), n^{\prime}=u^{\prime}\left(x^{\prime}, s^{\prime}, w^{\prime}\right)$ and $n_{k}=n(\xi, \sigma, \omega)$, this may be rewritten as

$$
\left(\begin{array}{c}
s \theta \\
-x
\end{array}\right)=\left(\begin{array}{cc}
a & b \theta \\
c \theta & d
\end{array}\right)^{-1}\left(\begin{array}{c}
s^{\prime} \\
-x^{\prime}(1+\theta)
\end{array}\right)+\left(\begin{array}{c}
\sigma \\
-\xi
\end{array}\right),
$$

for $s, s^{\prime}, x, x^{\prime} \in F$ and $\sigma, \xi \in \mathcal{O}_{E}$. Again, computing each entry, using rationality 
and integrality shows that $x^{\prime}, s^{\prime} \in \mathcal{O}_{F}$, so $n^{\prime} \in K_{0}$ and hence $n \in K_{0}$. Thus $q \in K_{0}$

(iv). The method here is identical to the previous step, except with the extra element $t_{0,1}$ included in the calculations. With $g, g^{\prime}, g_{k}$ as in (4.27), the equation now becomes

which implies that $g$ satisfies

$$
g=g^{\prime}\left(\begin{array}{cc}
\theta & \\
& \theta^{-1}
\end{array}\right) g_{k},
$$

$$
\begin{array}{ll}
a \in \theta^{2} \mathcal{O}_{F}, & b \in \mathcal{O}_{F}^{\times}, \\
c \in \theta^{-2} \mathcal{O}_{F}^{\times}, & d \in \mathcal{O}_{F},
\end{array}
$$

which we abbreviate by

$$
g \in\left\{\left(\begin{array}{cc}
\theta^{2} \mathcal{O}_{F} & \theta \mathcal{O}_{F}^{\times} \\
\theta^{-1} \mathcal{O}_{F}^{\times} & \mathcal{O}_{F}
\end{array}\right)\right\} .
$$

The unipotent equation (4.26) likewise becomes $n v=\left(t m_{k}\right)^{-1} n^{\prime}\left(t m_{k}\right) n_{k}$, which leaves (4.28) as before. (Here we do not assume $n^{\prime}=n_{1}^{\prime}$.) Using (4.30) and proceeding as before we find that $v \in K_{0}$ and

$$
x \in \mathcal{O}_{F}, \quad s \in \theta^{-2} \mathcal{O}_{F},
$$

with $x$ and $s$ as in (4.29). Thus we may (symbolically) write

$$
q \in\left\{\left(\begin{array}{cccc}
\mathcal{O}_{F}^{\times} & \mathcal{O}_{F} & \theta \mathcal{O}_{F} & \theta^{-1} \mathcal{O}_{F} \\
& \theta^{2} \mathcal{O}_{F} & \theta \mathcal{O}_{F} & \theta \mathcal{O}_{F}^{\times} \\
& & \mathcal{O}_{F}^{\times} & \\
& \theta^{-1} \mathcal{O}_{F}^{\times} & \theta^{-2} \mathcal{O}_{F} & \mathcal{O}_{F}
\end{array}\right)\right\} \subseteq Q(F) \cap t_{\theta} K_{0} t_{\theta}^{-1}
$$


where

$$
t_{\theta}=h\left(\theta^{-1}, \theta, \theta^{2}, 1\right) .
$$

One immediate consequence of (iii) and (iv) is that

$$
\left.\Xi_{s}\right|_{\left(Q X_{2}^{Q^{\prime}}\right)(F) \cap \widetilde{N}^{Q^{\prime}}(F) t_{0, \epsilon} K_{0}} \equiv 1
$$

since for $g$ in either set, both $e(g)$ and $e^{\prime}(g)$ are units.

Now we may define $f^{*}$ as follows. Let $K_{0}^{\prime}$ be the open compact subgroup of $K_{0}$ chosen above, and choose an open compact subgroup $K_{1}^{\prime} \subseteq K_{0}$ such that $t_{\theta} K_{1}^{\prime} t_{\theta}^{-1} \subseteq K_{0}^{\prime}$. Then $\left(Q X_{2}^{Q^{\prime}}\right)(F) \cap K_{0}^{\prime}$ has finite index in $\left(Q X_{2}^{Q^{\prime}}\right)(F) \cap K_{0}$; let $\left\{k_{i, 0}\right\}_{i=1}^{r_{0}}$ represent the quotient and set $\Omega_{0}=\cup_{i=1}^{r_{0}} k_{i, 0} K_{0}^{\prime}$, so $\Omega_{0}$ is an open compact subset of $K_{0}$. Similarly, let $\left\{k_{i, 1}\right\}_{i=1}^{r_{1}}$ represent the (finite) quotient of $\left(Q X_{2}^{Q^{\prime}}\right)(F) \cap t_{\theta} K_{0} t_{\theta}^{-1}$ by $\left(Q X_{2}^{Q^{\prime}}\right)(F) \cap t_{\theta} K_{1}^{\prime} t_{\theta}^{-1}$ and set $\Omega_{1}=\cup_{i=1}^{r_{1}} k_{i, 1} t_{\theta} K_{1}^{\prime} t_{\theta}^{-1}$. Define

$$
\begin{aligned}
& \left.f^{*}\right|_{\widetilde{N}^{Q^{\prime}(F) t_{0,0} K_{0}}}=\mathbf{1}_{\Omega_{0}}, \\
& \left.f^{*}\right|_{\tilde{N} Q^{\prime}(F) t_{0,1} K_{0}}=\mathbf{1}_{\Omega_{1}} .
\end{aligned}
$$

Then by (iii) and (iv) of Lemma 4.2.4,

$$
\left.f^{*}\right|_{\left(Q X_{2}^{Q^{\prime}}\right)(F) \cap \widetilde{N}^{Q^{\prime}}(F) t_{0, \epsilon} K_{0}} \equiv 1
$$

by choice of $\Omega_{1}, \Omega_{2}$, and

$$
\operatorname{supp} f^{*} \subseteq\left(Q X_{2}^{Q^{\prime}}\right)(F) \cdot K_{0}^{\prime} \cup\left(Q X_{2}^{Q^{\prime}}\right)(F) \cdot t_{\theta} K_{1}^{\prime} t_{\theta}^{-1} \subseteq\left(Q X_{2}^{Q^{\prime}}\right)(F) \cdot K_{0}^{\prime},
$$

by choice of $K_{1}^{\prime}$. Finally we have $\left.f^{*}\right|_{K_{0}^{\prime}} \equiv 1$ by definition. This proves the claim. 


\subsubsection{Archimedean case}

Since we have restricted our attention to nonsplit places, we have $F=\mathbb{R}, E=\mathbb{C}$, and we can take $\theta^{2}=-1$. Also, it is easy to check that

$$
\begin{gathered}
N_{4}(\mathbb{C}) \cap K_{0}=1, \\
T_{4}(\mathbb{C}) \cap K_{0}=T_{4}(\mathbb{C})^{1},
\end{gathered}
$$

where $K_{0}=\operatorname{Sp}(4)^{ \pm}$as in $(4.8)$. Here $T_{4}(\mathbb{C})^{1}$ is the subgroup of $T_{4}(\mathbb{C})$ with entries in $\mathbb{C}^{1}$ (=complex numbers of modulus 1$)$. Similarly we denote by $T_{4}(\mathbb{R})_{+}^{\times}$the subgroup of $T_{4}(\mathbb{R})$ whose elements have positive real entries.

We have a similar group decomposition to (4.19), with the modification that since $T_{4}(\mathbb{C})=T_{4}(\mathbb{R})_{+}^{\times} \cdot T_{4}(\mathbb{C})^{1}$, we have $T_{4}(\mathbb{C})^{\circ} K_{0}=T_{4}(\mathbb{R})_{+}^{\times} \cdot K_{0}=T_{4}(F) K_{0}$ and so

$$
G_{0}(F)=\left(Q X_{2}^{Q^{\prime}}\right)(F) \cdot \tilde{N}^{Q^{\prime}}(F) K_{0}
$$

The isomorphism (4.12) also holds, and because $\widetilde{N}^{Q^{\prime}}(F) \cap K_{0}=1$, we can choose the section $f^{*}$ to be given by

$$
f^{*}\left(q^{\prime} k\right)=\phi_{1}\left(q^{\prime}\right) \phi_{2}(k), \quad \text { for } q^{\prime} \in \widetilde{N}^{Q^{\prime}}(F), k \in K_{0},
$$

where $\phi_{1} \in C_{c}^{\infty}\left(\widetilde{N}^{Q^{\prime}}(F)\right)$ and $\phi_{2} \in C_{c}^{\infty}\left(K_{0}\right)$ will be determined below. Note that if $f_{s}$ corresponds to $f^{*}$ under $(4.12)$, then $f_{s}$ will not be $K_{8}$-finite: a $K_{8}$-finite function will be real analytic, hence cannot vanish on an open set.

Recall our integral

$$
\Psi\left(s, W, f_{s}\right)=\int_{Z(F) X_{2}^{Q^{\prime}}(F) N^{Q}(F) \backslash G_{0}(F)} f_{s}\left(\gamma_{0} g\right) W(g) d g .
$$


Proposition 4.2.5. Let $\sigma_{0}$ be as in Prop. 4.1.8. Then the integral $\Psi\left(s, W, f_{s}\right)$ is not identically zero for $\operatorname{Re}(s)>\sigma_{0}$. More precisely, there is a choice of $K_{4}$-finite Whittaker function $W_{0}$ and smooth section $f_{s, 0}$ such that $\Psi\left(s, W_{0}, f_{s, 0}\right)$ is not zero, independent of $s$, for $\operatorname{Re}(s)>\sigma_{0}$.

To show this we need some preliminary results, as before.

First consider the integral

$$
J(s, g, \widetilde{W})=\int_{N_{2}(F) \backslash P_{2}^{\prime}(F)}|\operatorname{det} p|^{s} \widetilde{W}(m(p g)) d p ;
$$

this is exactly the integral (4.13) and it converges for $\operatorname{Re}(s)>>0$ by the same argument. Also note that $J(s, g, \widetilde{W})$ will be $K_{2}$-finite when $\widetilde{W}$ is $K_{4}$-finite.

Lemma 4.2.6. We may choose $\widetilde{W}$ such that $J(s, 1, \widetilde{W})>0$ for all $s$ with $\operatorname{Re}(s)$ sufficiently large.

Proof. The choice of $\widetilde{W}$ and the formal manipulation of the integral proceed exactly as in Lemma 4.2 .2 , noting that $\widetilde{W}$ will be $K_{4}$-finite if $\widetilde{W}^{\prime}$ is. We only need to note that since $\Phi^{\prime} \in C_{c}^{\infty}(F)$, its Fourier transform $\hat{\Phi}^{\prime}$ is in $\mathcal{S}(F)$, hence is rapidly decreasing. So by the moderate growth of $\widetilde{W}^{\prime}$, the integral (4.14) defining $\widetilde{W}$ is absolutely convergent. Unfolding the integral and choosing $\Phi^{\prime}$ nonnegative with small support (and independent of $s$ ) as before, we note that though the integrand is no longer constant on the support of $\Phi^{\prime}$, the integral will still be positive for all $s$ with $\operatorname{Re}(s)$ sufficiently large. This completes the argument.

Next we examine the integral given by

$$
A(s, y, k ; W, \Phi)=\int_{N_{2}(F) \backslash \mathrm{GL}_{2}(F)}|e(g)|^{s} W\left(m(g) u^{\prime \prime}(y) k\right) \Phi((0,1) g) d g
$$


for $y \in F, k \in K_{0}, \Phi \in C_{c}^{\infty}\left(F^{2}\right)$ and $W$ a Whittaker function. This converges for $\operatorname{Re}(s)>>0$ by the gauge estimate, Prop. 4.1.3.

Lemma 4.2.7. Given $s$ with $\operatorname{Re}(s)$ large enough, there are $W$ and $\Phi$ such that $A(s, 1,1 ; W, \Phi)>0$.

Proof. The steps are roughly the same as in the proof of Lemma 4.2.3. Decomposing $\mathrm{GL}_{2}(F)=P_{2}^{\prime}(F) Z(F) K_{2}$ with $K_{2}=\mathrm{O}(2)$ and the measure $d g=\delta_{B_{2}}^{-1}(p z) d p d z d k$, we see that

$$
\begin{aligned}
A(s, 1,1 ; W, \Phi) & =\int_{K_{2}} \int_{Z_{2}(F)} \int_{N_{2}(F) \backslash P_{2}^{\prime}(F)}|\operatorname{det} p|^{s-1}|\operatorname{det} z|^{s} \\
& \cdot W(m(p z k)) \Phi((0,1) z k) d p d z d k \\
& =\int_{K_{2}} \int_{F^{\times}}|a|^{2 s} J(s-1, a k, W) \Phi((0,1) a k) d^{\times} a d k,
\end{aligned}
$$

where we have identified $z=\left(\begin{array}{c}{ }^{a} \\ a\end{array}\right)$ with the scalar $a \in F^{\times}$. Take $W$ as in Lemma 4.2.6 so that $J(s-1,1, W)=1$, and let

$$
\Phi(u, v)= \begin{cases}\Phi((0,1) a k) \stackrel{\text { def }}{=} \phi_{3}(a) \overline{J(s-1, k, W)} & \text { if }(u, v) \neq(0,0) \\ 0 & \text { if }(u, v)=(0,0)\end{cases}
$$

for $\phi_{3} \in C_{c}^{\infty}\left(F^{\times}\right)$to be chosen. Since for $(u, v) \neq(0,0)$ we have

$$
(u, v)=(0,1) a k=(0,1) a k_{\alpha}=(a \sin \alpha, a \cos \alpha)
$$

and $u^{2}+v^{2}=a^{2}, \Phi$ is supported on an annulus (with positive inner radius) about the origin; it is $K_{2}$-finite, hence smooth in $k$, since $J$ is $K_{2}$-finite. So $\Phi \in C_{c}^{\infty}\left(F^{2}\right)$. The integral in question now becomes

$$
A(s, 1,1 ; W, \Phi)=\int_{F^{\times}}|a|^{2 s} \phi_{3}(a) \int_{K_{2}} J(s-1, a k, W) \overline{J(s-1, k, W)} d k d^{\times} a .
$$


If we denote the inner integral by $A_{1}(s, a)$, then $A_{1}(s, 1)>0$. Thus we may take $\phi_{3}$ to have support containing 1 and small enough that $A(s, 1,1 ; W, \Phi) \neq 0$.

Proof of Prop. 4.2.5. Let $f^{*}$ be as in (4.32) and let $f_{s, 0}$ correspond to $f^{*}$ under (4.12). Then

$$
\begin{aligned}
& \Psi\left(s, W, f_{s, 0}\right)=\int_{Z(F) X_{2}^{Q^{\prime}}(F) N^{Q}(F) \backslash G_{0}(F)} f^{*}(g) W(g) d g \\
& =\int_{\{ \pm I\} \backslash K_{0}} \int_{X_{2}^{Q^{\prime}}(F) \backslash N^{Q^{\prime}}(F)} \int_{Z(F) N^{Q}(F) \backslash Q(F)} f^{*}\left(q q^{\prime} k\right) W\left(q q^{\prime} k\right) d q d q^{\prime} d k \\
& =\int_{\bar{K}_{0}} \phi_{2}(k) \int_{X_{2}^{Q^{\prime}}(F) \backslash N^{Q^{\prime}}(F)} \phi_{1}\left(q^{\prime}\right) \int_{N_{2}(F) \backslash \mathrm{GL}_{2}(F)}|e(g)|^{s^{\prime}} W\left(m(g) q^{\prime} k\right) d g d q^{\prime} d k,
\end{aligned}
$$

where $\bar{K}_{0}=\{ \pm I\} \backslash K_{0}$ and $s^{\prime}=\frac{s+3}{2}$. Now let $q^{\prime}$ have Levi decomposition $q^{\prime}=n^{\prime} m^{\prime}=$ $u^{\prime}(x, z) u^{\prime \prime}(y)$, for $n^{\prime}=u^{\prime}(x, z)=u^{\prime}(x, z, 0) \in U^{Q^{\prime}}(F)$ and $m^{\prime}=u^{\prime \prime}(y) \in Q^{\prime}(F)$. As varieties, $X_{2}^{Q^{\prime}}(F) \backslash N^{Q^{\prime}}(F) \simeq F^{3}$, so we may take $\phi_{1}$ to be $\phi_{1}\left(q^{\prime}\right)=\phi_{1}\left(n^{\prime} m^{\prime}\right)=$ $\phi_{1}^{\prime}(x, z) \phi_{1}^{\prime \prime}(y)$ for $\phi_{1}^{\prime} \in C_{c}^{\infty}\left(F^{2}\right)$ and $\phi_{1}^{\prime \prime} \in C_{c}^{\infty}(F)$ to be chosen. The integral becomes

$$
\begin{aligned}
\Psi\left(s, W, f_{s, 0}\right)=\int_{\bar{K}_{0}} \phi_{2}(k) & \int_{X_{2}^{Q^{\prime}}(F) \backslash N^{Q^{\prime}}(F)} \phi_{1}\left(n^{\prime} m^{\prime}\right) \\
& \int_{N_{2}(F) \backslash \mathrm{GL}_{2}(F)}|e(g)|^{s^{\prime}} W\left(m(g) n^{\prime} m^{\prime} k\right) d g d\left(n^{\prime} m^{\prime}\right) d k \\
=\int_{\bar{K}_{0}} \phi_{2}(k) \int_{F} \phi_{1}^{\prime \prime}(y) & \int_{N_{2}(F) \backslash \mathrm{GL}_{2}(F)}|e(g)|^{s^{\prime}} W\left(m(g) u^{\prime \prime}(y) k\right) \\
& \left(\int_{F^{2}} \phi_{1}^{\prime}(x, z) \psi\left(\operatorname{Ad}(m(g)) u^{\prime}(x, z)\right) d x d z\right) d g d y d k .
\end{aligned}
$$

(Note that although $d\left(n^{\prime} m^{\prime}\right)=\delta_{P_{4}}^{-1}\left(m^{\prime}\right) d n^{\prime} d m^{\prime}, m^{\prime}=u^{\prime \prime}(y)$ has unipotent image in $\mathrm{GL}_{2}(E)$ under $(4.23)$ and so $\delta_{P_{4}}\left(u^{\prime \prime}(y)\right)=1$.) By $(4.15)$ and $(4.18)$, the integral over $x$ and $z$ is

$$
\int_{F^{2}} \phi_{1}^{\prime}(x, z) \psi_{\circ}\left(\theta^{2}(c z+d x)\right) d x d z, \quad g=\left(\begin{array}{ll}
a & b \\
c & d
\end{array}\right)
$$


which is the Fourier transform of $\phi_{1}^{\prime}$. Hence we may choose $\phi_{1}^{\prime}$ so that the integral equals $\Phi(c, d)$ for our choice of $\Phi$ in Lemma 4.2.7, and for $(c, d) \neq(0,0)$ we may write $\Phi(c, d)=\Phi((0,1) g)$. This yields

$$
\begin{aligned}
\Psi\left(s, W, f_{s, 0}\right) & =\int_{\bar{K}_{0}} \phi_{2}(k) \int_{F} \phi_{1}^{\prime \prime}(y) \int_{N_{2}(F) \backslash \mathrm{GL}_{2}(F)}|e(g)|^{s^{\prime}} \\
& \cdot W\left(m(g) u^{\prime \prime}(y) k\right) \Phi((0,1) g) d g d y d k \\
& =\int_{\bar{K}_{0}} \phi_{2}(k) \int_{F} \phi_{1}^{\prime \prime}(y) A\left(s^{\prime}, y, k ; W, \Phi\right) d y d k,
\end{aligned}
$$

with $A\left(s^{\prime}, y, k ; W, \Phi\right)$ given by (4.33). By Lemma 4.2.7, there is a Whittaker function $W_{0}$ such that $A\left(s^{\prime}, 1,1 ; W_{0}, \Phi\right)>0$. So choose $\phi_{1}^{\prime \prime} \geq 0$ and $\phi_{2} \geq 0$ with support containing 1 and small enough that $A\left(s^{\prime}, y, k ; W_{0}, \Phi\right)>0$ on $\operatorname{supp} \phi_{1}^{\prime \prime} \times \operatorname{supp} \phi_{2}$. Then the inner integral is positive for $k \in \operatorname{supp} \phi_{2}$, so $\Psi\left(s, W_{0}, f_{s, 0}\right)>0$ as well.

\subsection{Final Remarks}

Before concluding we would like to return to consideration of the global integral (2.5). After this, we outline our continuing work with this integral and highlight some possible future directions of research.

By [24, IV, Prop. 1.1.2], the Eisenstein series $E^{*}\left(g, s, f_{s}\right)$ has simple poles at $s=3$ and $s=2$ and a double pole at $s=1$. Since the meromorphic continuation of the

series propagates to that of the integral, studying poles of the integral $I^{*}\left(s, \varphi, f_{s}\right) \stackrel{\text { def }}{=}$ $d_{G}(s) I\left(s, \varphi, f_{s}\right)$ amounts to studying residues of this Eisenstein series. Jiang carries this out in chapter 4 of [24], and the result is as follows. 
Proposition 4.3.1 (cf. [24], IV, Thm. 4.0.2). The integral $I^{*}\left(s, \varphi, f_{s}\right)$ is holomorphic for all s except possibly at $s=1$, where it may achieve a pole of order at most two.

Proof. We only need to check holomorphy at $s=3$ and $s=2$, and Jiang's proof carries over with minimal modification. We only outline the argument. At $s=3$, the residue of $E^{*}\left(g, s, f_{s}\right)$ is constant, so the residue is

$$
\operatorname{Res}_{s=3} I^{*}\left(s, \varphi, f_{s}\right)=c_{3} \int_{Z(\mathbb{A}) G_{0}(F) \backslash G_{0}(\mathbb{A})} \varphi(g) d g,
$$

which vanishes since $\varphi$ is a cusp form.

At $s=2$, Jiang proves an identity of between the residue of $E^{*}\left(g, s, f_{s}\right)$ and the constant term of an Eisenstein series $E^{1, *}\left(g, s, M\left(f_{s}\right)\right)$ on the (standard) parabolic subgroup $P_{8}^{1}$ of $\mathrm{Sp}_{8}$ with Levi factor isomorphic to $\mathrm{Sp}_{6} \times \mathrm{GL}_{1}$, where $M$ is an intertwining operator. So the residue at $s=2$ is

$$
\operatorname{Res}_{s=2} I^{*}\left(s, \varphi, f_{s}\right)=c_{2} \int_{Z(\mathbb{A}) G_{0}(F) \backslash G_{0}(\mathbb{A})} \varphi(g) E^{1, *}\left(g, s, M\left(f_{s}\right)\right) d g .
$$

Here the integral vanishes after unfolding the Eisenstein series, because all the orbits of $G_{0}(F)$ on $P_{8}^{1}(F) \backslash \mathrm{Sp}_{8}(F)$ are negligible (in the sense of [35]).

This leaves the question of behavior of the integral at $s=1$. In order to motivate the discussion that follows, first recall that Jiang's global integral is

$$
\tilde{I}^{*}\left(s, \varphi_{1}, \varphi_{2}, f_{s}\right)=\int_{Z(\mathbb{A}) \widetilde{G}_{0}(F) \backslash \widetilde{G}_{0}(\mathbb{A})} \varphi_{1}\left(g_{1}\right) \varphi_{2}\left(g_{2}\right) E^{*}\left(\left(g_{1}, g_{2}\right), s, f_{s}\right) d\left(g_{1}, g_{2}\right)
$$

where $\varphi_{1}$ and $\varphi_{2}$ are cusp forms belonging to (irreducible, smooth, generic) cuspidal automorphic representations $\pi_{1}$ and $\pi_{2}$ of $\mathrm{GSp}_{4}\left(\mathbb{A}_{F}\right)$, respectively. Jiang shows [24, IV, Thm. 3.2.1] another identity between the second-order residue of $E^{*}\left(g, s, f_{s}\right)$ at 
$s=1$ and the first-order residue of the Siegel-type Eisenstein series $E_{8}^{4, *}\left(g, s, M^{\prime}\left(f_{s}\right)\right)$ on $\mathrm{Sp}_{8}$ at $s=\frac{1}{2}$. So his integral has residue at $s=1$

$$
\begin{aligned}
& \operatorname{Res}_{s=1} \tilde{I}^{*}\left(s, \varphi_{1}, \varphi_{2}, f_{s}\right) \\
& \quad=c_{1} \cdot \operatorname{Res}{ }_{s=\frac{1}{2}} \int_{Z(\mathbb{A}) \widetilde{G}_{0}(F) \backslash \widetilde{G}_{0}(\mathbb{A})} \varphi_{1}\left(g_{1}\right) \varphi_{2}\left(g_{2}\right) E_{8}^{4, *}\left(g, s, M^{\prime}\left(f_{s}\right)\right) d\left(g_{1}, g_{2}\right) .
\end{aligned}
$$

But the integral on the right is the doubling integral of Piatetski-Shapiro and Rallis, which is nonzero if and only if $\pi_{1}$ and $\pi_{2}$ are contragredient [35, pp. 3-6]. Furthermore, by [31, Thm. 7.2.5], if the doubling integral has a pole at $s=\frac{1}{2}$, then $\pi_{1}=\check{\pi}_{2}$ has a nonvanishing theta lift to $\mathrm{GO}_{2,2}$. This is Thm. 4.0.3, chapter IV, in [24].

Now we return to the integral $I^{*}\left(s, \varphi, f_{s}\right)$. Repeating the above analysis and using the same identities of Eisenstein series, we arrive at

$$
\operatorname{Res}_{s=1} I^{*}\left(s, \varphi, f_{s}\right)=c_{1}^{\prime} \cdot \operatorname{Res}_{s=\frac{1}{2}} \int_{Z(\mathbb{A}) G_{0}(F) \backslash G_{0}(\mathbb{A})} \varphi(g) E_{8}^{4, *}\left(g, s, f_{s}\right) d g .
$$

The integral on the right is a kind of "twisted doubling" integral, and it can be shown that this integral is nonvanishing if and only if the period

$$
\mathcal{P}(\varphi)=\int_{Z(\mathbb{A}) \operatorname{GSp}_{4}(F) \backslash \mathrm{GSp}_{4}(\mathbb{A})} \varphi(g) d g
$$

is nonzero for some $\varphi \in V_{\pi}$. By analogy with Jiang's case, we pose the question: does the nonvanishing of this period indicate that $\pi$ is a base change (functorial) lift from an automorphic representation $\pi_{0}$ on $\operatorname{GSp}_{4}\left(\mathbb{A}_{F}\right)$ ? Some evidence or at least heuristic in the affirmative direction is found in $[16,14]$. Furthermore, $\pi$ is a functorial lift from some $\pi_{0}$, we may also ask if the existence of a pole of the "twisted doubling" integral is indicative of $\pi_{0}$ having a nonvanishing theta lift to a certain orthogonal group. The author intends to pursue these questions in later research. 
Another set of questions the author is currently researching concerns the local integrals of Chapter 4. We have already mentioned that in the archimedean case, we need to show nonvanishing of the integrals for $K$-finite data, not just smooth data. This will likely be done using a continuity result and density argument similar to that in [7]. We also would like to show all the local integrals can be meromorphically continued to the complex plane. This is a standard argument in the nonarchimedean case (cf. [39]), and ultimately relies on asymptotic expansions for the Whittaker functions in the archimedean case (see [40]). It is useful because it allows us to relate poles of the global integral back to poles of the global $L$-function, which could then give a criterion for $\pi$ begin a functorial lift in terms of poles of the twisted tensor $L$-function. However, more precise information is needed for this: we would need to know not only the continuation of the local integrals, but also that the ratio of local integral to local $L$-factor,

$$
e_{v}\left(s, W_{v}, f_{s, v}\right)=\frac{\Psi_{v}^{*}\left(s, W_{v}, f_{s, v}\right)}{L_{v}\left(s, \pi_{v}, \rho_{\mathrm{TT}}\right)}
$$

is entire and that for any $s, W_{v}$ and $f_{s, v}$ can be chosen to make the ratio nonzero. This is subtle and difficult, though conceivable since the $L$-factor is already known at archimedean places, and is now known at nonarchimedean places thanks to recent work of Gan and Takeda [9]. The global argument would then be based on the 
equality

$$
\begin{aligned}
I\left(s, \varphi, f_{s}\right) & =\prod_{v \in \Sigma_{F}} \Psi_{v}^{*}\left(s, W_{v}, f_{s, v}\right) \\
& =\left(\prod_{v \in S} \Psi_{v}^{*}\left(s, W_{v}, f_{s, v}\right)\right) \prod_{v \notin S} L_{v}\left(s, \pi_{v}, \rho_{\mathrm{TT}}\right) \\
& =\left(\prod_{v \in S} \frac{\Psi_{v}^{*}\left(s, W_{v}, f_{s, v}\right)}{L_{v}\left(s, \pi_{v}, \rho_{\mathrm{TT}}\right)}\right) \prod_{v \in \Sigma_{F}} L_{v}\left(s, \pi_{v}, \rho_{\mathrm{TT}}\right) \\
& =\left(\prod_{v \in S} e_{v}\left(s, W_{v}, f_{s, v}\right)\right) L\left(s, \pi, \rho_{\mathrm{TT}}\right) .
\end{aligned}
$$

Since there are $W_{v}$ and $f_{s, v}$ for $v \in S$ such that the product of the factors $e_{v}\left(s, W_{v}, f_{s, v}\right)$ is entire and nonzero, $I^{*}\left(s, \varphi, f_{s}\right)$ and $L\left(s, \pi, \rho_{\mathrm{TT}}\right)$ would have the same set of poles to the same orders. This would allow us to pose the above questions in terms of poles of $L\left(s, \pi, \rho_{\mathrm{TT}}\right)$, rather than those of the global integral. 


\section{BIBLIOGRAPHY}

[1] J. Arthur, Eisenstein series and the trace formula, in Automorphic forms, representations and L-functions (Proc. Sympos. Pure Math., Oregon State Univ., Corvallis, Ore., 1977), Part 1, Proc. Sympos. Pure Math., XXXIII, pp. 253274, Amer. Math. Soc., Providence, R.I. (1979).

[2] A. Borel, Automorphic L-functions, in Automorphic forms, representations and L-functions (Proc. Sympos. Pure Math., Oregon State Univ., Corvallis, Ore., 1977), Part 2, Proc. Sympos. Pure Math., XXXIII, pp. 27-61, Amer. Math. Soc., Providence, R.I. (1979).

[3] D. Bump, The Rankin-Selberg method: an introduction and survey, in Automorphic representations, L-functions and applications: progress and prospects, volume 11 of Ohio State Univ. Math. Res. Inst. Publ., pp. 41-73, de Gruyter, Berlin (2005).

[4] W. Casselman, Canonical extensions of Harish-Chandra modules to representations of $G$, Canad. J. Math., 41 (1989), no. 3, 385-438.

[5] W. Casselman and J. Shalika, The unramified principal series of p-adic groups. II. The Whittaker function, Compositio Math., 41 (1980), no. 2, 207-231.

[6] J. W. Cogdell, Lectures on L-functions, converse theorems, and functoriality for $\mathrm{GL}_{n}$, in Lectures on automorphic L-functions, volume 20 of Fields Inst. Monogr., pp. 1-96, Amer. Math. Soc., Providence, RI (2004).

[7] J. W. Cogdell and I. I. Piatetski-Shapiro, Remarks on Rankin-Selberg convolutions, in Contributions to automorphic forms, geometry, and number theory, pp. 255-278, Johns Hopkins Univ. Press, Baltimore, MD (2004).

[8] Y. Z. Flicker, Twisted tensors and Euler products, Bull. Soc. Math. France, 116 (1988), no. 3, 295-313. 
[9] W. T. Gan and S. Takeda, The local Langlands conjecture for GSp(4) (2007). Available at http://www.math.ucsd.edu/ wgan/ or at http://arxiv.org (arXiv:0706.0952v3 [math.NT]).

[10] S. Gelbart and I. Piatetski-Shapiro, Automorphic forms and L-functions for the unitary group, in Lie group representations, II (College Park, Md., 1982/1983), volume 1041 of Lecture Notes in Math., pp. 141-184, Springer, Berlin (1984).

[11] S. Gelbart and I. Piatetski-Shapiro, L-functions for $G \times \operatorname{GL}(n)$, pp. 53-146, volume 1254 of Lecture Notes in Mathematics [12] (1987). Part B.

[12] S. Gelbart, I. Piatetski-Shapiro and S. Rallis, Explicit constructions of automorphic L-functions, volume 1254 of Lecture Notes in Mathematics, Springer-Verlag, Berlin (1987).

[13] R. Goodman and N. R. Wallach, Representations and invariants of the classical groups, volume 68 of Encyclopedia of Mathematics and its Applications, Cambridge University Press, Cambridge (1998).

[14] N. Gurevich, The relative trace formula for groups with involution, Israel J. Math., 121 (2001), 125-141.

[15] H. Jacquet and R. P. Langlands, Automorphic forms on GL(2), volume 114 of Lecture Notes in Mathematics, Springer-Verlag, Berlin (1970).

[16] H. Jacquet, E. Lapid and J. Rogawski, Periods of automorphic forms, J. Amer. Math. Soc., 12 (1999), no. 1, 173-240.

[17] H. Jacquet, I. I. Piatetski-Shapiro and J. Shalika, Automorphic forms on GL(3). I, Ann. of Math. (2), 109 (1979), no. 1, 169-212.

[18] H. Jacquet, I. I. Piatetski-Shapiro and J. Shalika, Automorphic forms on GL(3). II, Ann. of Math. (2), 109 (1979), no. 2, 213-258.

[19] H. Jacquet, I. I. Piatetskii-Shapiro and J. A. Shalika, Rankin-Selberg convolutions, Amer. J. Math., 105 (1983), no. 2, 367-464.

[20] H. Jacquet and J. Shalika, Exterior square L-functions, in Automorphic forms, Shimura varieties, and L-functions, Vol. II (Ann Arbor, MI, 1988), volume 11 of Perspect. Math., pp. 143-226, Academic Press, Boston, MA (1990). 
[21] H. Jacquet and J. Shalika, Rankin-Selberg convolutions: Archimedean theory, in Festschrift in honor of I. I. Piatetski-Shapiro on the occasion of his sixtieth birthday, Part I (Ramat Aviv, 1989), volume 2 of Israel Math. Conf. Proc., pp. 125-207, Weizmann, Jerusalem (1990).

[22] H. Jacquet and J. A. Shalika, On Euler products and the classification of automorphic forms. II, Amer. J. Math., 103 (1981), no. 4, 777-815.

[23] H. Jacquet and J. A. Shalika, On Euler products and the classification of automorphic representations. I, Amer. J. Math., 103 (1981), no. 3, 499-558.

[24] D. Jiang, Degree 16 standard L-function of $\operatorname{GSp}(2) \times \operatorname{GSp}(2)$, Mem. Amer. Math. Soc., 123 (1996), no. 588, viii+196.

[25] D. Jiang, On the fundamental automorphic L-functions of $\mathrm{SO}(2 n+1)$, Int. Math. Res. Not., (2006), Art. ID 64069, 26.

[26] D. Jiang and D. Soudry, The multiplicity-one theorem for generic automorphic forms of GSp(4), Pacific J. Math., 229 (2007), no. 2, 381-388.

[27] A. W. Knapp, Lie groups beyond an introduction, volume 140 of Progress in Mathematics, Birkhäuser Boston Inc., Boston, MA, second edition (2002).

[28] K. Koike and I. Terada, Young-diagrammatic methods for the representation theory of the classical groups of type $B_{n}, C_{n}, D_{n}$, J. Algebra, 107 (1987), no. 2, 466-511.

[29] B. Kostant and S. Rallis, Orbits and representations associated with symmetric spaces, Amer. J. Math., 93 (1971), 753-809.

[30] S. S. Kudla, Seesaw dual reductive pairs, in Automorphic forms of several variables (Katata, 1983), volume 46 of Progr. Math., pp. 244-268, Birkhäuser Boston, Boston, MA (1984).

[31] S. S. Kudla and S. Rallis, A regularized Siegel-Weil formula: the first term identity, Ann. of Math. (2), 140 (1994), no. 1, 1-80.

[32] R. P. Langlands, Problems in the theory of automorphic forms, in Lectures in modern analysis and applications, III, pp. 18-61. Lecture Notes in Math., Vol. 170, Springer, Berlin (1970). 
[33] R. P. Langlands, On the functional equations satisfied by Eisenstein series, Lecture Notes in Mathematics, Vol. 544, Springer-Verlag, Berlin (1976).

[34] I. G. Macdonald, Symmetric functions and Hall polynomials, Oxford Mathematical Monographs, The Clarendon Press Oxford University Press, New York, second edition (1995). With contributions by A. Zelevinsky, Oxford Science Publications.

[35] I. Piatetski-Shapiro and S. Rallis, L-functions for the classical groups, pp. 1-52, volume 1254 of Lecture Notes in Mathematics [12] (1987). Part A.

[36] I. I. Piatetski-Shapiro, Multiplicity one theorems, in Automorphic forms, representations and L-functions (Proc. Sympos. Pure Math., Oregon State Univ., Corvallis, Ore., 1977), Part 1, Proc. Sympos. Pure Math., XXXIII, pp. 209212, Amer. Math. Soc., Providence, R.I. (1979).

[37] I. I. Pjateckij-Šapiro, Euler subgroups, in Lie groups and their representations (Proc. Summer School, Bolyai János Math. Soc., Budapest, 1971), pp. 597620, Halsted, New York (1975).

[38] J. A. Shalika, The multiplicity one theorem for $\mathrm{GL}_{n}$, Ann. of Math. (2), 100 (1974), 171-193.

[39] D. Soudry, Rankin-Selberg convolutions for $\mathrm{SO}_{2 l+1} \times \mathrm{GL}_{n}$ : local theory, Mem. Amer. Math. Soc., 105 (1993), no. 500, vi+100.

[40] D. Soudry, On the Archimedean theory of Rankin-Selberg convolutions for $\mathrm{SO}_{2 l+1} \times$ $\mathrm{GL}_{n}$, Ann. Sci. École Norm. Sup. (4), 28 (1995), no. 2, 161-224.

[41] S. Sundaram, On the Combinatorics of Representations of $\operatorname{Sp}(2 n, \mathbb{C}), \mathrm{Ph} . \mathrm{D}$. thesis, Massachusetts Institute of Technology (1986).

[42] S. Sundaram, Tableaux in the representation theory of the classical Lie groups, in Invariant theory and tableaux (Minneapolis, MN, 1988), volume 19 of IMA Vol. Math. Appl., pp. 191-225, Springer, New York (1990).

[43] J. Tate, Number theoretic background, in Automorphic forms, representations and L-functions (Proc. Sympos. Pure Math., Oregon State Univ., Corvallis, Ore., 1977), Part 2, Proc. Sympos. Pure Math., XXXIII, pp. 3-26, Amer. Math. Soc., Providence, R.I. (1979). 